\title{
LONG TIME DYNAMICS OF NON-RADIAL SOLUTIONS TO INHOMOGENEOUS NONLINEAR SCHRÖDINGER EQUATIONS
}

\author{
VAN DUONG DINH AND SAHBI KERAANI
}

\begin{abstract}
We study long time dynamics of non-radial solutions to the focusing inhomogeneous nonlinear Schrödinger equation. By using the concentration/compactness and rigidity method, we establish a scattering criterion for non-radial solutions to the equation. We also prove a non-radial blow-up criterion for the equation whose proof makes use of localized virial estimates. As a byproduct of these criteria, we study long time dynamics of non-radial solutions to the equation with data lying below, at, and above the ground state threshold. In addition, we provide a new argument showing the existence of finite time blow-up solution to the equation with cylindrically symmetric data. The ideas developed in this paper are robust and can be applicable to other types of nonlinear Schrödinger equations.
\end{abstract}

\section{INTRODUCTION}

The nonlinear Schrödinger equation (NLS) is one of the most important equations in nonlinear optics. It models the propagation of intense laser beams in a homogeneous bulk medium with a Kerr nonlinearity. It is well-known that NLS governed the beam propagation cannot support stable high-power propagation in a homogeneous bulk media. At the end of the last century, it was suggested that stable high-power propagation can be achieved in plasma by sending a preliminary laser beam that creates a channel with a reduced electron density, and thus reduces the nonlinear inside the channel (see e.g., [33,37]). Under these conditions, the beam propagation can be modeled by the inhomogeneous nonlinear Schrödinger equation of the form

$$
i \partial_{t} u+\Delta u+K(x)|u|^{\alpha} u=0, \quad(t, x) \in \mathbb{R} \times \mathbb{R}^{N},
$$

where $u$ is the electric field in laser and optics, $\alpha>0$ is the power of nonlinear interaction, and the potential $K(x)$ is proportional to the electron density. By means of variational approximation and direct simulations, Towers and Malomed [49] observed that for a certain type of nonlinear medium, (1.1) gives rise to completely stable beams.

The equation (1.1) has been attracted a lot of interest from the mathematical community. When the potential $K(x)$ is constant, (1.1) is the usual nonlinear Schrödinger equation which has been studied extensively in the past decades (see e.g., the monographs $[7,45,47]$ ).

In the case of non-constant bounded potential $K(x)$, Merle [40] proved the existence and nonexistence of minimal blow-up solutions to (1.1) with $\alpha=\frac{4}{N}$ and $K_{1} \leq K(x) \leq K_{2}$, where $K_{1}$ and $K_{2}$ are positive constants. Based on the work of Merle, Raphaël and Szeftel [43] established sufficient conditions for the existence, uniqueness, and charaterization of minimial blow-up solutions to the equation. Fibich and Wang [24], and Liu and Wang [38] investigated the stability and instability of solitary waves for (1.1) with $\alpha \geq \frac{4}{N}$ and $K(x)=K(\epsilon x)$, where $\epsilon>0$ is a small parameter and $K \in C^{4}\left(\mathbb{R}^{N}\right) \cap L^{\infty}\left(\mathbb{R}^{N}\right)$.

When the potential $K(x)$ is unbounded, the problem becomes more subtle. The case $K(x)=|x|^{b}, b>0$ was studied in several works, for instance, Chen and Guo [9], and Chen [8] established sharp criteria for the global existence and blow-up, and Zhu [52] studied the existence and dynamical properties of blow-up solutions. When $K(x)$ behaves like $|x|^{-b}$ with $b>0$, De Bouard and Fukuizumi [3] studied the stability of standing waves for (1.1) with $\alpha<\frac{4-2 b}{N}$. Fukuizumi and Ohta [26] established the instability of standing waves for (1.1) with $\alpha>\frac{4-2 b}{N}$ (see also [28,34] and references therein for other studies related to standing waves for this type of equation).

In this paper, we consider the Cauchy problem for a class of focusing inhomogeneous nonlinear Schrödinger equations (INLS)

$$
\left\{\begin{aligned}
i \partial_{t} u+\Delta u & =-|x|^{-b}|u|^{\alpha} u, \quad(t, x) \in \mathbb{R} \times \mathbb{R}^{N}, \\
\left.u\right|_{t=0} & =u_{0} \in H^{1},
\end{aligned}\right.
$$

2010 Mathematics Subject Classification. 35Q44; 35Q55.

Key words and phrases. Inhomogeneous nonlinear Schrödinger equation; Global existence; Blow-up; Ground state. 
where $u: \mathbb{R} \times \mathbb{R}^{N} \rightarrow \mathbb{C}, u_{0}: \mathbb{R}^{N} \rightarrow \mathbb{C}, N \geq 1,0<b<\min \{2, N\}$, and $\frac{4-2 b}{N}<\alpha<\alpha(N)$ with

$$
\alpha(N):=\left\{\begin{array}{cll}
\frac{4-2 b}{N-2} & \text { if } \quad N \geq 3, \\
\infty & \text { if } \quad N=1,2 .
\end{array}\right.
$$

This equation plays an important role as a limiting equation in the analysis of (1.1) with $K(x) \sim|x|^{-b}$ as $|x| \rightarrow \infty$ (see e.g., [28,29]).

The local well-posedness for (1.2) was studied by Geneoud and Stuart [28, Appendix]. More precisely, they proved that (1.2) is locally well-posed in $H^{1}$ for $N \geq 1,0<b<\min \{2, N\}$, and $0<\alpha<\alpha(N)$. The proof of this result is based on the energy method developed by Cazenave [7], which does not use Strichartz estimates. See also [13,32] for other proofs based on Strichartz estimates and the contraction mapping argument. Note that the local well-posedness in $[13,32]$ is more restrictive than the one in [28]. However, it provides more information on the local solutions, for instance, local solutions belong to $L_{\mathrm{loc}}^{q}\left(\left(-T_{*}, T^{*}\right), W^{1, r}\left(\mathbb{R}^{N}\right)\right)$ for any Schrödinger admissible pair $(q, r)$ (see Section 2 for the definition of $L^{2}$ admissibility), where $\left(-T_{*}, T^{*}\right)$ is the maximal time interval of existence. Note that the latter property plays an important role in the scattering theory.

It is well-known that solutions to (1.2) satisfy the conservation laws of mass and energy

$$
\begin{aligned}
M(u(t)) & =\|u(t)\|_{L^{2}}^{2}=M\left(u_{0}\right), \\
E(u(t)) & =\frac{1}{2}\|\nabla u(t)\|_{L^{2}}^{2}-\frac{1}{\alpha+2} \int|x|^{-b}|u(t, x)|^{\alpha+2} d x=E\left(u_{0}\right) .
\end{aligned}
$$

The equation (1.2) also has the following scaling invariance

$$
u_{\lambda}(t, x):=\lambda^{\frac{2-b}{\alpha}} u\left(\lambda^{2} t, \lambda x\right), \quad \lambda>0 .
$$

A direct calculation gives

$$
\left\|u_{\lambda}(0)\right\|_{\dot{H}^{\gamma}}=\lambda^{\gamma+\frac{2-b}{\alpha}-\frac{N}{2}}\left\|u_{0}\right\|_{\dot{H}^{\gamma}}
$$

which shows that (1.4) leaves the $\dot{H}^{\gamma_{\mathrm{c}}}$-norm of initial data invariant, where

$$
\gamma_{\mathrm{c}}:=\frac{N}{2}-\frac{2-b}{\alpha} \text {. }
$$

The condition $\frac{4-2 b}{N}<\alpha<\alpha(N)$ is equivalent to $0<\gamma_{\mathrm{c}}<1$ which corresponds to the mass-supercritical and energy-subcritical range (intercritical range, for short). For later uses, it is convenient to introduce the following exponent

$$
\sigma_{\mathrm{c}}:=\frac{1-\gamma_{\mathrm{c}}}{\gamma_{\mathrm{c}}}=\frac{4-2 b-(N-2) \alpha}{N \alpha-4+2 b} .
$$

The main purpose of the present paper is to study long time dynamics (global existence, energy scattering, and finite time blow-up) of non-radial solutions to (1.2). Before stating our contributions, let us recall known results related to dynamics of (1.2) in the intercritical range.

In [20], Farah showed the global existence for (1.2) with $N \geq 1$ and $0<b<\min \{2, N\}$ by assuming $u_{0} \in H^{1}$ and

$$
\begin{aligned}
& E\left(u_{0}\right)\left[M\left(u_{0}\right)\right]^{\sigma_{\mathrm{c}}}<E(Q)[M(Q)]^{\sigma_{\mathrm{c}}}, \\
& \left\|\nabla u_{0}\right\|_{L^{2}}\left\|u_{0}\right\|_{L^{2}}^{\sigma_{\mathrm{c}}}<\|\nabla Q\|_{L^{2}}\|Q\|_{L^{2}}^{\sigma_{\mathrm{c}}},
\end{aligned}
$$

where $Q$ is the unique postive radial solution to the elliptic equation

$$
-\Delta Q+Q-|x|^{-b}|Q|^{\alpha} Q=0 .
$$

He also proved the finite time blow-up for (1.2) with $u_{0} \in \Sigma:=H^{1} \cap L^{2}\left(|x|^{2} d x\right)$ satisfying (1.7) and

$$
\left\|\nabla u_{0}\right\|_{L^{2}}\left\|u_{0}\right\|_{L^{2}}^{\sigma_{c}}>\|\nabla Q\|_{L^{2}}\|Q\|_{L^{2}}^{\sigma_{\mathrm{c}}}
$$

The latter result was extended to radial data by the first author in [11]. Note that the uniqueness of positive radial solution to (1.9) was established by Yanagida [51] for $N \geq 3$, Genoud [29] for $N=2$, and Toland [48] for $N=1$.

The energy scattering (or asymptotic behavior) for (1.2) was first established by Farah and Guzmán [21] with $0<b<\frac{1}{2}, \alpha=2, N=3$, and radial data. The proof of this result is based on the concentration/compactness and rigidity argument introduced by Kenig and Merle [36]. This scattering result was later extended to dimensions $N \geq 2$ in [22] by using the same concentration/compactness and rigidity method.

Later, Campos [4] made use of a new idea of Dodson and Murphy [16] to give an alternative simple proof for the radial scattering results of Farah and Guzmán. He also extends the validity of $b$ in dimensions 
$N \geq 3$. Note that the idea of Dodson and Murphy is a combination of a scattering criterion of Tao [46], localized virial estimates, and radial Sobolev embedding.

Afterwards, Xu and Zhao [50], and the first author [14] have simultaneously showed the energy scattering for (1.2) with $0<b<1, N=2$, and radial data. The proof relies on a new approach of Arora, Dodson, and Murhpy [1], which is a refined version of the one in [16].

In [5], Campos and Cardoso studied long time dynamics such as global existence, energy scattering, and finite time blow-up of $H^{1}$-solutions to (1.2) with data in $\Sigma$ lying above the ground state threshold.

Recently, Miao, Murphy, and Zheng [41] showed a new nonlinear profile for non-radial solutions related to (1.2). In particular, they constructed nonlinear profiles with data living far away from the origin. This allows them to show the energy scattering of non-radial solution to (1.2) with $0<b<\frac{1}{2}$, $\alpha=2$, and $N=3$. This result was extended to any dimensions $N \geq 2$ and $0<b<\min \left\{2, \frac{N}{2}\right\}$ by Cardoso, Farah, Guzmán, and Murphy [6].

We also mention the works $[12,14]$ for the energy scattering for the defocusing problem INLS and [10] for the energy scattering for the focusing energy-critical INLS.

Motivated by the aforementioned works, we study the global existence, energy scattering, and finite time blow-up of non-radial solutions to (1.2). To this end, let us start with the following scattering criterion for (1.2).

Theorem 1.1 (Scattering criterion). Let $N \geq 1,0<b<\min \{2, N\}$, and $\frac{4-2 b}{N}<\alpha<\alpha(N)$. Let $u$ be a solution to (1.2) defined on the maximal forward time interval of existence $\left[0, T^{*}\right)$. Assume that

$$
\sup _{t \in\left[0, T^{*}\right)} P(u(t))[M(u(t))]^{\sigma_{\mathrm{c}}}<P(Q)[M(Q)]^{\sigma_{\mathrm{c}}},
$$

where

$$
P(f):=\int|x|^{-b}|f(x)|^{\alpha+2} d x .
$$

Then $T^{*}=\infty$. Moreover, if we assume in addition that $N \geq 2$ and $0<b<\min \left\{2, \frac{N}{2}\right\}$, then the solution scatters in $H^{1}$ forward in time, i.e., there exists $u_{+} \in H^{1}$ such that

$$
\lim _{t \rightarrow \infty}\left\|u(t)-e^{i t \Delta} u_{+}\right\|_{H^{1}}=0
$$

A similar statement holds for negative times.

We note that a scattering condition similar to (1.11) was first introduced by Duyckaerts and Roudenko in [19, Theorem 3.7], where it was used to show the scattering beyond the ground state threshold for the focusing Schrödinger equation. The condition (1.11) was inspired by a recent work of Gao and Wang [27] (see also [15]).

The proof of Theorem 1.1 is based on the concentration/compactness and rigidity method. The main difficulty comes from the fact that the potential energy $P(u(t))$ is not conserved along the time evolution of (1.2). To overcome the difficulty, we establish a Pythagorean expansion along bounded nonlinear flows. Since we are interested in non-radial solutions, we need to construct nonlinear profiles associated with the linear ones living far away from the origin. The latter was recently showed by Miao, Murphy, and Zheng [41] in three dimensions (see also [6] for dimensions $N \geq 2$ ). This type of nonlinear profiles is constructed by observing that in the regime $|x| \rightarrow \infty$, the nonlinearity becomes weak, and solutions to (1.2) can be approximated by solutions to the underlying linear Schrödinger equation. Thanks to an improved nonlinear estimate (see Lemma 2.2), we give a refined result with a simple proof of these results (see Lemma 2.7). For more details, we refer to Section 2.

Our next result is the following blow-up criterion for (1.2).

Theorem 1.2 (Blow-up criterion). Let $N \geq 1,0<b<\min \{2, N\}$, and $\frac{4-2 b}{N}<\alpha<\alpha(N)$. Let $u$ be a solution to (1.2) defined on the maximal forward time interval of existence $\left[0, T^{*}\right)$. Assume that

$$
\sup _{t \in\left[0, T^{*}\right)} G(u(t)) \leq-\delta
$$

for some $\delta>0$, where

$$
G(f):=\|\nabla f\|_{L^{2}}^{2}-\frac{N \alpha+2 b}{2(\alpha+2)} P(f) .
$$

Then either $T^{*}<\infty$, or $T^{*}=\infty$ and there exists a time sequence $t_{n} \rightarrow \infty$ such that $\left\|\nabla u\left(t_{n}\right)\right\|_{L^{2}} \rightarrow \infty$ as $n \rightarrow \infty$. Moreover, if we assume in addition that $u$ has finite variance, i.e., $|x| u(t) \in L^{2}\left(|x|^{2} d x\right)$ for all $t \in\left[0, T^{*}\right)$, then $T^{*}<\infty$. A similar statement holds for negative times. 
The proof of this blow-up result is based on a contradiction argument using localized virial estimates for general (non-radial and infinite variance) solutions to (1.2) (see Lemma 3.2). We also take the advantage of the decay of the nonlinear term outside a large ball. It is conjectured that if a general (not finite variance or radially symmetric) solution to (1.2) satisfy (1.14), then it blows up in finite time. However, there is no affirmative answer for this conjecture up to date even for the classical nonlinear Schrödinger equation.

A first application of Theorems 1.1 and 1.2 is the following long time dynamics below the ground state threshold.

Theorem 1.3 (Dynamics below the ground state threshold). Let $N \geq 1,0<b<\min \{2, N\}$, and $\frac{4-2 b}{N}<\alpha<\alpha(N)$. Let $u_{0} \in H^{1}$ satisfy (1.7).

(1) If $u_{0}$ satisfies (1.8), then the corresponding solution to (1.2) satisfies

$$
\sup _{t \in\left(-T_{*}, T^{*}\right)} P(u(t))[M(u(t))]^{\sigma_{\mathrm{c}}}<P(Q)[M(Q)]^{\sigma_{\mathrm{c}}} .
$$

In particular, the solution exists globally in time. Moreover, if we assume in addition that $N \geq 2$ and $0<b<\min \left\{2, \frac{N}{2}\right\}$, then the corresponding solution scatters in $H^{1}$ in both directions.

(2) If $u_{0}$ satisfies (1.10), then the corresponding solution to (1.2) satisfies

$$
\sup _{t \in\left(-T_{*}, T^{*}\right)} G(u(t)) \leq-\delta
$$

for some $\delta>0$. In particular, the solution either blows up in finite time, or there exists a time sequence $\left(t_{n}\right)_{n \geq 1}$ satisfying $\left|t_{n}\right| \rightarrow \infty$ such that $\left\|\nabla u\left(t_{n}\right)\right\|_{L^{2}} \rightarrow \infty$ as $n \rightarrow \infty$. Moreover, if we assume in addition that

- $u_{0}$ has finite variance,

- or $N \geq 2, \alpha \leq 4$, and $u_{0}$ is radially symmetric,

- or $N \geq 3, \alpha \leq 2$, and $u_{0} \in \Sigma_{N}$, where

$$
\Sigma_{N}:=\left\{f \in H^{1}: f\left(y, x_{N}\right)=f\left(|y|, x_{N}\right), x_{N} f \in L^{2}\right\}
$$

with $x=\left(y, x_{N}\right), y=\left(x_{1}, \cdots, x_{N-1}\right) \in \mathbb{R}^{N-1}$, and $x_{N} \in \mathbb{R}$,

then the corresponding solution blows up in finite time, i.e., $T_{*}, T^{*}<\infty$.

For the scattering part, Theorem 1.3 provides an alternative proof of a recent result of Cardoso, Farah, Guzmán, and Murphy [6]. For the blow-up part, Theorem 1.3 extends earlier results of [20] (for finite variance data) and the first author [11] (for radial data) to the case of cylindrically symmetric data. Note that the first work addressed the finite time blow-up for NLS with cylindrically symmetric data is due to Martel [39], where the blow-up was shown for data with negative energy. Recently, Bellazzini and Forcella [2] extended Martel's result to the case of focusing cubic NLS for data with non-negative energy data lying below the ground state threshold. Our result not only extends the ones of [2,39] to the focusing inhomogeneous NLS but also provides an alternative simple proof for these results. In particular, our choice of cutoff function is simpler than that in [2,39]. Our argument is robust and can be applied to show the existence of finite time blow-up solutions with cylindrically symmetric data for other Schrödinger-type equations.

Another application of Thereorems (1.1) and (1.2) is the following long time dyanmics at the ground state threshold.

Theorem 1.4 (Dynamics at the ground state). Let $N \geq 1,0<b<\min \{2, N\}$, and $\frac{4-2 b}{N}<\alpha<\alpha(N)$. Let $u_{0} \in H^{1}$ be such that

(1) If

$$
E\left(u_{0}\right)\left[M\left(u_{0}\right)\right]^{\sigma_{\mathrm{c}}}=E(Q)[M(Q)]^{\sigma_{\mathrm{c}}}
$$

$$
\left\|\nabla u_{0}\right\|_{L^{2}}\left\|u_{0}\right\|_{L^{2}}^{\sigma_{\mathrm{c}}}<\|\nabla Q\|_{L^{2}}\|Q\|_{L^{2}}^{\sigma_{\mathrm{c}}}
$$

then the corresponding solution to (1.2) exists globally in time. Moreover, the solution either satisfies

$$
\sup _{t \in \mathbb{R}} P(u(t))[M(u(t))]^{\sigma_{\mathrm{c}}}<P(Q)[M(Q)]^{\sigma_{\mathrm{c}}}
$$

or there exists a time sequence $\left(t_{n}\right)_{n \geq 1}$ satisfying $\left|t_{n}\right| \rightarrow \infty$ such that

$$
u\left(t_{n}\right) \rightarrow e^{i \theta} Q \quad \text { strongly in } H^{1}
$$

for some $\theta \in \mathbb{R}$ as $n \rightarrow \infty$. In particular, if we we assume in addition that $N \geq 2$ and $0<b<\min \left\{2, \frac{N}{2}\right\}$, then the solution either scatters in $H^{1}$ forward in time, or there exist a time sequence $t_{n} \rightarrow \infty$ and a sequence $\left(x_{n}\right)_{n \geq 1} \subset \mathbb{R}^{N}$ such that (1.22) holds. 
(2) If

$$
\left\|\nabla u_{0}\right\|_{L^{2}}\left\|u_{0}\right\|_{L^{2}}^{\sigma_{c}}=\|\nabla Q\|_{L^{2}}\|Q\|_{L^{2}}^{\sigma_{c}}
$$

then $u(t, x)=e^{i t} e^{i \theta} Q(x)$ for some $\theta \in \mathbb{R}$.

(3) If

$$
\left\|\nabla u_{0}\right\|_{L^{2}}\left\|u_{0}\right\|_{L^{2}}^{\sigma_{c}}>\|\nabla Q\|_{L^{2}}\|Q\|_{L^{2}}^{\sigma_{c}}
$$

then the corresponding solution to (1.2)

i. either blows up forward in time, i.e., $T^{*}<\infty$,

ii. or there exists a time sequence $t_{n} \rightarrow \infty$ such that $\left\|\nabla u\left(t_{n}\right)\right\|_{L^{2}} \rightarrow \infty$ as $n \rightarrow \infty$,

iii. or there exists a time sequence $t_{n} \rightarrow \infty$ such that (1.22) holds.

Moreover, if we assume in addition that

- $u_{0}$ has finite variance,

- or $N \geq 2, \alpha \leq 4$, and $u_{0}$ is radially symmetric,

- or $N \geq 3, \alpha \leq 2$, and $u_{0} \in \Sigma_{N}$,

then the possibility in Item ii. can be excluded.

To our knowledge, Theorem 1.4 is the first result addressing long time dynamics of solutions to (1.2) with data lying at the ground state threshold. For the classical NLS, dynamics at the ground state threshold was first studied by Duyckaerts and Roudenko [18] for the 3D focusing cubic NLS. The proof in [18] relies on delicate spectral estimates which make it difficult to extend to higher dimensions. Recently, the first author in [15] gave a simple approach to study the dynamics at the threshold for the focusing NLS in any dimensions. Our result is an extension of the one in [15] to the focusing inhomogeneous NLS. The proof of Theorem 1.4 relies on the scattering and blow-up criteria given in Theorems 1.1, 1.2, and the compactness property of optimizing sequence for the Gagliardo-Nirenberg inequality (2.3) (see Lemma 4.2). We refer the reader to Section 4 for more details.

Finally, we study long time dynamics above the ground state threshold. Before stating our result, we introduce the virial quantity

$$
V(t):=\int|x|^{2}|u(t, x)|^{2} d x
$$

If $V(0)<\infty$, then $V(t)<\infty$ for all $t$ in the existence time. Moreover, the following identities hold

$$
\begin{aligned}
V^{\prime}(t) & =4 \operatorname{Im} \int \bar{u}(t, x) x \cdot \nabla u(t, x) d x, \\
V^{\prime \prime}(t) & =8\|\nabla u(t)\|_{L^{2}}^{2}-\frac{4(N \alpha+2 b)}{\alpha+2} P(u(t)) .
\end{aligned}
$$

Theorem 1.5 (Dynamics above the ground state). Let $N \geq 1,0<b<\min \{2, N\}$, and $\frac{4-2 b}{N}<\alpha<$ $\alpha(N)$. Let $u_{0} \in \Sigma$ satisfy

(1) If

$$
\begin{aligned}
& E\left(u_{0}\right)\left[M\left(u_{0}\right)\right]^{\sigma_{\mathrm{c}}} \geq E(Q)[M(Q)]^{\sigma_{\mathrm{c}}}, \\
& \frac{E\left(u_{0}\right)\left[M\left(u_{0}\right)\right]^{\sigma_{\mathrm{c}}}}{E(Q)[M(Q)]^{\sigma_{\mathrm{c}}}}\left(1-\frac{\left(V^{\prime}(0)\right)^{2}}{32 E\left(u_{0}\right) V(0)}\right) \leq 1 .
\end{aligned}
$$

$$
\begin{aligned}
P\left(u_{0}\right)\left[M\left(u_{0}\right)\right]^{\sigma_{c}} & <P(Q)[M(Q)]^{\sigma_{c}}, \\
V^{\prime}(0) & \geq 0,
\end{aligned}
$$

then the corresponding solution to (1.2) satisfies (1.11). In particular, if $N \geq 2$ and $0<b<\min \left\{2, \frac{N}{2}\right\}$, then the solution exists globally in time and scatters in $H^{1}$ in the sense of (1.13).

(2) If

$$
\begin{aligned}
P\left(u_{0}\right)\left[M\left(u_{0}\right)\right]^{\sigma_{c}} & >P(Q)[M(Q)]^{\sigma_{c}}, \\
V^{\prime}(0) & \leq 0,
\end{aligned}
$$

then the corresponding solution to (1.2) blows up forward in time, i.e., $T^{*}<\infty$.

For the scattering part, Theorem 1.5 improves a recent result of Campos and Cardoso [5] at two points: (1) removing the radial assumption and (2) extending the validity of $b$. For the blow-up part, we extend the one in [5] to any dimensions $N \geq 1$. The proof of Theorem 1.5 is based on virial identities and a continuity argument in the same spirit of Duyckaerts and Roudenko [19]. 
We finish the introduction by outlining the structure of the paper. In Section 2, we give the proof of the scattering criterion given in Theorem 1.1. In Section 3, we prove the blow-up criterion given in Theorem 1.2. Finally, we study long time dynamics of $H^{1}$-solutions lying below, at, and above the ground state threshold in Section 4.

\section{SCATtering CRIterion}

2.1. Local theory. In this subsection, we recall the well-posedness theory for (1.2) due to [21, 22, 32]. To this end, we introduce some notations. Let $\gamma \geq 0$. A pair $(q, r)$ is called $\dot{H}^{\gamma}$-admissible if

$$
\frac{2}{q}+\frac{N}{r}=\frac{N}{2}-\gamma
$$

and

$$
\left\{\begin{array}{ccc}
\frac{2 N}{N-2 \gamma}<r<\frac{2 N}{N-2} & \text { if } & N \geq 3 \\
\frac{2}{1-\gamma}<r<\infty & \text { if } & N=2 \\
\frac{2}{1-2 \gamma}<r<\infty & \text { if } & N=1
\end{array}\right.
$$

The set of all $\dot{H}^{\gamma}$-admissible pairs is denoted by $\mathcal{A}_{\gamma}$. Similarly, a pair $(q, r)$ is called $\dot{H}^{-\gamma}$-admissible if

$$
\frac{2}{q}+\frac{N}{r}=\frac{N}{2}+\gamma
$$

and $r$ satisfies (2.1). The set of all $\dot{H}^{-\gamma}$-admissible pairs is denoted by $\mathcal{A}_{-\gamma}$. Note that we do not consider the pair $\left(\infty, \frac{2 N}{N-2 \gamma}\right)$ as a $\dot{H}^{\gamma}$-admissible pair. The reason for doing so will be clear in Subsection 2.3. When $\gamma=0$, we denote $L^{2}$ instead of $\dot{H}^{0}$. In this case, the $L^{2}$-admissible pair is also called Schrödinger admissible.

Let $I \subset \mathbb{R}$ be an interval and $\gamma \geq 0$. We define the Strichartz norm

$$
\|u\|_{S\left(I, \dot{H}^{\gamma}\right)}:=\sup _{(q, r) \in \mathcal{A}_{\gamma}}\|u\|_{L_{t}^{q}\left(I, L_{x}^{r}\right)}
$$

For a set $A \subset \mathbb{R}^{N}$, we denote

$$
\|u\|_{S\left(I, \dot{H}^{\gamma}(A)\right)}:=\sup _{(q, r) \in \mathcal{A}_{\gamma}}\|u\|_{L_{t}^{q}\left(I, L_{x}^{r}(A)\right)}
$$

When $I=\mathbb{R}$, we omit the dependence on $\mathbb{R}$ and simply denote $\|u\|_{S\left(\dot{H}^{\gamma}\right)}$ and $\|u\|_{S\left(\dot{H}^{\gamma}(A)\right)}$. Similarly, we define

and for $A \subset \mathbb{R}^{N}$,

$$
\|u\|_{S^{\prime}\left(I, \dot{H}^{-\gamma}\right)}:=\inf _{(q, r) \in \mathcal{A}_{-\gamma}}\|u\|_{L_{t}^{q^{\prime}}\left(I, L_{x}^{r^{\prime}}\right)}
$$

$$
\|u\|_{S^{\prime}\left(I, \dot{H}^{-\gamma}(A)\right)}:=\inf _{(q, r) \in \mathcal{A}_{-\gamma}}\|u\|_{L_{t}^{q^{\prime}}\left(I, L_{x}^{r^{\prime}}(A)\right)} .
$$

As before, when $I=\mathbb{R}$, we simply use $\|u\|_{S^{\prime}\left(\dot{H}^{-\gamma}\right)}$ and $\|u\|_{S^{\prime}\left(\dot{H}^{-\gamma}(A)\right)}$.

We have the following Strichartz estimates (see e.g., [7, 25,35]).

Proposition 2.1 (Strichartz estimates [7,25,35]). Let $\gamma \geq 0$ and $I \subset \mathbb{R}$ be an interval. Then there exists a constant $C>0$ independent of $I$ such that

and

$$
\left\|e^{i t \Delta} f\right\|_{S\left(I, \dot{H}^{\gamma}\right)} \leq C\|f\|_{\dot{H}^{\gamma}}
$$

$$
\left\|\int_{0}^{t} e^{i(t-s) \Delta} F(s) d s\right\|_{S\left(I, \dot{H}^{\gamma}\right)} \leq C\|F\|_{S^{\prime}\left(I, \dot{H}^{-\gamma}\right)} .
$$

Moreover, the above estimates still hold with $L_{t}^{\infty}\left(I, L_{x}^{\frac{2 N}{N-2 \gamma}}\right)$-norm in place of $S\left(I, \dot{H}^{\gamma}\right)$-norm.

We also need the following nonlinear estimates due to [4, Lemma 2.5] and [6, Lemma 2.1].

Lemma 2.2 (Nonlinear estimates [4,6]). Let $N \geq 2,0<b<\min \left\{2, \frac{N}{2}\right\}$, and $\frac{4-2 b}{N}<\alpha<\alpha(N)$. Then there exists $\theta \in(0, \alpha)$ sufficiently small so that

$$
\begin{aligned}
\left\||x|^{-b}|u|^{\alpha} v\right\|_{S^{\prime}\left(\dot{H}^{-} \gamma_{c}\right)} & \lesssim\|u\|_{L_{t}^{\infty} H_{x}^{1}}^{\theta}\|u\|_{S\left(\dot{H} \gamma_{c}\right)}^{\alpha-\theta}\|v\|_{S\left(\dot{H}^{\gamma_{c}}\right)}, \\
\left\||x|^{-b}|u|^{\alpha} v\right\|_{S^{\prime}\left(L^{2}\right)} & \lesssim\|u\|_{L_{t}^{\infty} H_{x}^{1}}^{\theta}\|u\|_{S\left(\dot{H} \gamma_{c}\right)}^{\alpha-\theta}\|v\|_{S\left(L^{2}\right)}, \\
\left\|\nabla\left(|x|^{-b}|u|^{\alpha} u\right)\right\|_{S^{\prime}\left(L^{2}\right)} & \lesssim\|u\|_{L_{t}^{\infty} H_{x}^{1}}^{\theta}\|u\|_{S\left(\dot{H} \gamma_{c}\right)}^{\alpha-\theta}\|\nabla u\|_{S\left(L^{2}\right)} .
\end{aligned}
$$


Note that if $b=0$, we can take $\theta=0$ in the above estimates.

Proof. The first two estimates were proved in [4, Lemma 2.5] (for $N \geq 3$ ) and [6, Lemma 2.1] (for $N \geq 2$ ). An estimate similar to the last one was proved in [4, Lemma 2.5] for $N \geq 3$. However, the proof in [4] used the dual pair of the end-point $\left(2, \frac{2 N}{N-2}\right)$ which, however, is excluded in our definition of $L^{2}$-admissible pair (see (2.1)). Thus we need a different argument. Let $\theta>0$ be a small parameter to be chosen later. We denote

$$
\begin{aligned}
q^{\prime} & =\frac{4}{2+\theta}, & r^{\prime} & =\frac{2 N}{N+2-\theta}, \\
\bar{a} & =\frac{4 \alpha(\alpha+1-\theta)}{4-2 b-(N-2) \alpha+\theta \alpha}, & \bar{r} & =\frac{2 N \alpha(\alpha+1-\theta)}{(N+2-2 b) \alpha-\theta(4-2 b+\alpha)}, \\
\bar{q} & =\frac{4 \alpha(\alpha+1-\theta)}{\alpha(N \alpha-2+2 b)-\theta(N \alpha-4+2 b-\alpha)}, & \bar{m}_{ \pm} & =\frac{N \alpha}{2-b \mp N \alpha \theta} .
\end{aligned}
$$

Here $\left(q^{\prime}, r^{\prime}\right)$ is the dual pair of $\left(\frac{4}{2-\theta}, \frac{2 N}{N-2+\theta}\right) \in \mathcal{A}_{0}$. We can readily check that $(\bar{q}, \bar{r}) \in \mathcal{A}_{0}$ and $(\bar{a}, \bar{r}) \in \mathcal{A}_{\gamma_{\mathrm{c}}}$ provided that $\theta>0$ is taken sufficiently small. Moreover, as $\frac{4-2 b}{N}<\alpha<\frac{4-2 b}{N-2}$, we have $2<\bar{m}_{ \pm}<\frac{2 N}{N-2}$ for $\theta>0$ sufficiently small.

We observe that

$$
\nabla\left(|x|^{-b}|u|^{\alpha} u\right)=|x|^{-b} \nabla\left(|u|^{\alpha} u\right)-b \frac{x}{|x|}|x|^{-b}\left(|x|^{-1}|u|^{\alpha} u\right)
$$

and

$$
\left\||x|^{-b} f\right\|_{L_{x}^{r^{\prime}(A)}} \leq\left\||x|^{-b}\right\|_{L_{x}^{r_{1}(A)}}\|f\|_{L_{x}^{r_{2}}}
$$

where $A$ stands for either $B=B(0,1)$ or $B^{c}=\mathbb{R}^{N} \backslash B(0,1)$. To ensure $\left\||x|^{-b}\right\|_{L_{x}^{r_{1}(A)}}<\infty$, we take

$$
\frac{1}{r_{1}}=\frac{b}{N} \pm \theta^{2}
$$

where the plus sign is for $A=B$ and the minus one is for $A=B^{c}$. It follows that

$$
\frac{1}{r_{2}}=\frac{1}{r^{\prime}}-\frac{1}{r_{1}}=\frac{N+2-2 b-\theta}{2 N} \mp \theta^{2} \text {. }
$$

As $\frac{1}{N}<\frac{N+2-2 b}{2 N}<1$ for $N \geq 2$ and $0<b<\frac{N}{2}$, we choose $\theta>0$ sufficiently small so that $1<r_{2}<N$ which allows us to use the Hardy's inequality (see e.g., [42])

$$
\left\||x|^{-1} f\right\|_{L_{x}^{r_{2}}} \leq \frac{r_{2}}{N-r_{2}}\|\nabla f\|_{L_{x}^{r_{2}}} .
$$

Applying the above inequality to $f=|u|^{\alpha} u$ and using (2.2), we see that

$$
\left\|\nabla\left(|x|^{-b}|u|^{\alpha} u\right)\right\|_{L_{x}^{r^{\prime}}} \lesssim\left\|\nabla\left(|u|^{\alpha} u\right)\right\|_{L_{x}^{r_{2}}}
$$

By Hölder's inequality and the fact that

$$
\frac{1}{r_{2}}=\frac{\theta}{\bar{m}_{ \pm}}+\frac{\alpha+1-\theta}{\bar{r}},
$$

we have

$$
\left\|\nabla\left(|x|^{-b}|u|^{\alpha} u\right)\right\|_{L_{x}^{r^{\prime}}} \lesssim\|u\|_{L_{x}^{\bar{m}_{ \pm}}}^{\theta}\|u\|_{L_{x}^{\bar{r}}}^{\alpha-\theta}\|\nabla u\|_{L_{x}^{\bar{r}}} .
$$

By Hölder's inequality in time with

$$
\frac{1}{q^{\prime}}=\frac{\alpha-\theta}{\bar{a}}+\frac{1}{\bar{q}}
$$

we get

$$
\begin{aligned}
\left\|\nabla\left(|x|^{-b}|u|^{\alpha} u\right)\right\|_{L_{t}^{q^{\prime}} L_{x}^{r^{\prime}}} & \lesssim\|u\|_{L_{t}^{\infty} L_{x}^{\bar{m}}}^{\theta}\|u\|_{L_{t}^{\bar{a}} L_{x}^{\bar{\tau}}}^{\alpha-\theta}\|\nabla u\|_{L_{t}^{\bar{q}} L_{x}^{\bar{r}}} \\
& \lesssim\|u\|_{L_{t}^{\infty} H_{x}^{1}}^{\theta}\|u\|_{L_{t}^{\bar{a}} L_{x}^{\bar{\tau}}\|\nabla u\|_{L_{t}^{\bar{q}} L_{x}^{\bar{\tau}}}}
\end{aligned}
$$

where the last inequality follows from the Sobolev embedding as $2<\bar{m}_{ \pm}<\frac{2 N}{N-2}$. The proof is complete.

Using Proposition 2.1 and Lemma 2.2, we have the following result.

Proposition 2.3 (Local theory [21,22,32]). Let $N \geq 2,0<b<\min \left\{2, \frac{N}{2}\right\}$, and $\frac{4-2 b}{N}<\alpha<\alpha(N)$. 
(1) (Local well-posedness) Let $u_{0} \in H^{1}$. Then there exist $T_{*}, T^{*} \in(0, \infty]$, and a unique local solution to (1.2) satisfying

$$
\left.u \in C\left(\left(-T_{*}, T^{*}\right), H^{1}\right) \cap L_{\mathrm{loc}}^{q}\left(-T_{*}, T^{*}\right), W^{1, r}\right)
$$

for any $(q, r) \in \mathcal{A}_{0}$. If $T^{*}<\infty$ (resp. $\left.T_{*}<\infty\right)$, then $\lim _{t \nearrow T^{*}}\|\nabla u(t)\|_{L^{2}}=\infty\left(\right.$ resp. $\lim _{t \searrow-T_{*}}\|\nabla u(t)\|_{L^{2}}=$ $\infty)$.

(2) (Small data scattering) Let $T>0$ be such that $\|u(T)\|_{H^{1}} \leq A$ for some constant $A>0$. Then there exists $\delta=\delta(A)>0$ such that if

$$
\left\|e^{i(t-T) \Delta} u(T)\right\|_{S\left([T, \infty), \dot{H}^{\gamma_{c}}\right)}<\delta
$$

then the corresponding solution to (1.2) with initial data $\left.u\right|_{t=T}=u(T)$ exists globally in time and satisfies

$$
\begin{aligned}
\|u\|_{S\left([T, \infty), \dot{H}^{\gamma_{c}}\right)} & \leq 2\left\|e^{i(t-T) \Delta} u(T)\right\|_{S\left([T, \infty), \dot{H}^{\gamma_{c}}\right)}, \\
\|\langle\nabla\rangle u\|_{S\left([T, \infty), L^{2}\right)} & \leq C\|u(T)\|_{H^{1}} .
\end{aligned}
$$

(3) (Scattering condition) Let $u$ be a global solution to (1.2). Assume that

$$
\|u\|_{L_{t}^{\infty}\left(\mathbb{R}, H_{x}^{1}\right)} \leq A, \quad\|u\|_{S\left(\dot{H}^{\gamma_{c}}\right)}<\infty .
$$

Then $u$ scatters in $H^{1}$ in both directions.

Here we have used the following convention

$$
\|\langle\nabla\rangle f\|_{X}:=\|f\|_{X}+\|\nabla f\|_{X}, \quad f \in X .
$$

We also recall the following stability result due to [21,22].

Lemma 2.4 (Stability). Let $N \geq 2,0<b<\min \left\{2, \frac{N}{2}\right\}$, and $\frac{4-2 b}{N}<\alpha<\alpha(N)$. Let $0 \in I \subseteq \mathbb{R}$ and $\tilde{u}: I \times \mathbb{R}^{N} \rightarrow \mathbb{C}$ be a solution to

$$
i \partial_{t} \tilde{u}+\Delta \tilde{u}+|x|^{-b}|\tilde{u}|^{\alpha} \tilde{u}=e
$$

with $\left.\tilde{u}\right|_{t=0}=\tilde{u}_{0}$ satisfying

$$
\|\tilde{u}\|_{L_{t}^{\infty}\left(I, H_{x}^{1}\right)} \leq M, \quad\|\tilde{u}\|_{S\left(I, \dot{H}^{\left.\gamma_{c}\right)}\right.} \leq L
$$

for some constants $M, L>0$. Let $u_{0} \in H^{1}$ be such that

$$
\left\|u_{0}-\tilde{u}_{0}\right\|_{H^{1}} \leq M^{\prime}, \quad\left\|e^{i t \Delta}\left(u_{0}-\tilde{u}_{0}\right)\right\|_{S\left(I, \dot{H} \gamma_{c}\right)} \leq \varepsilon
$$

for some $M^{\prime}>0$ and some $0<\varepsilon<\varepsilon_{1}=\varepsilon_{1}\left(M, M^{\prime}, L\right)$. Suppose that

$$
\|\langle\nabla\rangle e\|_{S^{\prime}\left(I, L^{2}\right)}+\|e\|_{S^{\prime}\left(I, \dot{H}^{-} \gamma_{c}\right)} \leq \varepsilon .
$$

Then there exists a unique solution $u: I \times \mathbb{R}^{N} \rightarrow \mathbb{C}$ to (1.2) with $\left.u\right|_{t=0}=u_{0}$ satisfying

$$
\begin{aligned}
\|u-\tilde{u}\|_{S\left(I, \dot{H}^{\gamma_{\mathrm{c}}}\right)} & \leq C\left(M, M^{\prime}, L\right) \varepsilon, \\
\|u\|_{L_{t}^{\infty}\left(I, H_{x}^{1}\right)}+\|\langle\nabla\rangle u\|_{S\left(I, L^{2}\right)}+\|u\|_{S\left(I, \dot{H}^{\gamma_{\mathrm{c}}}\right)} & \leq C\left(M, M^{\prime}, L\right) .
\end{aligned}
$$

Remark 2.1. If we assume in addition that

$$
\left\|e^{i t \Delta}\left(u_{0}-\tilde{u}_{0}\right)\right\|_{L_{t}^{\infty}\left(I, L_{x}^{\mathrm{N}} \frac{2 N}{2 \gamma_{\mathrm{c}}}\right)} \leq \varepsilon
$$

then

$$
\|u-\tilde{u}\|_{L_{t}^{\infty}\left(I, L_{x}^{N-2 \gamma_{c}}\right)} \leq C\left(M, M^{\prime}, L\right) \varepsilon .
$$

In fact, by Duhamel's formula, we have

$$
\begin{aligned}
u(t)-\tilde{u}(t)=e^{i t \Delta}\left(u_{0}-\tilde{u}_{0}\right) & +i \int_{0}^{t} e^{i(t-s) \Delta}\left(|x|^{-b}|u(s)|^{\alpha} u(s)-|x|^{-b}|\tilde{u}(s)|^{\alpha} \tilde{u}(s)\right) d s \\
& +i \int_{0}^{t} e^{i(t-s) \Delta} e(s) d s .
\end{aligned}
$$


By Strichartz estimates and Lemma 2.2, we have

$$
\begin{aligned}
\|u-\tilde{u}\|_{L_{t}^{\infty}\left(I, L_{x}^{\frac{2 N}{N-2 \gamma_{c}}}\right)} \leq & \left\|e^{i t \Delta}\left(u_{0}-\tilde{u}_{0}\right)\right\|_{L_{t}^{\infty}\left(I, L_{x}^{\frac{2 N}{N-2 \gamma_{c}}}\right)}+\|e\|_{S^{\prime}\left(I, \dot{H}^{-\gamma_{c}}\right)} \\
\leq & +C\left\||x|^{-b}|u|^{\alpha} u-|x|^{-b}|\tilde{u}|^{\alpha} \tilde{u}\right\|_{S^{\prime}\left(I, \dot{H}^{\gamma_{c}}\right)} \\
\leq & \left\|e^{i t \Delta}\left(u_{0}-\tilde{u}_{0}\right)\right\|_{L_{t}^{\infty}\left(I, L_{x}^{N-2 \gamma_{c}}\right)}+\|e\|_{S^{\prime}\left(I, \dot{H}^{-\gamma_{c}}\right)} \\
& +C\left(\|u\|_{L_{t}^{\infty}\left(I, H_{x}^{1}\right)}^{\theta}\|u\|_{S\left(I, \dot{H}^{\left.\gamma_{c}\right)}\right.}^{\alpha-\theta}+\|\tilde{u}\|_{L_{t}^{\infty}\left(I, H_{x}^{1}\right)}^{\theta}\|\tilde{u}\|_{S\left(I, \dot{H}^{\gamma_{c}}\right)}^{\alpha-\theta}\right)\|u-\tilde{u}\|_{S\left(I, \dot{H}^{\gamma_{c}}\right)} \\
\leq & C\left(M, M^{\prime}, L\right) \varepsilon .
\end{aligned}
$$

2.2. Variational analysis. We recall some properties of the ground state $Q$ which is the unique positive radial solution to (1.9). The ground state $Q$ optimizes the weighted Gagliardo-Nirenberg inequality: for $N \geq 1$ and $0<b<\min \{2, N\}$

$$
P(f) \leq C_{\mathrm{opt}}\|\nabla f\|_{L^{2}}^{\frac{N \alpha+2 b}{2}}\|f\|_{L^{2}}^{\frac{4-2 b-(N-2) \alpha}{2}}, \quad f \in H^{1}\left(\mathbb{R}^{N}\right),
$$

that is

$$
C_{\mathrm{opt}}=P(Q) \div\left[\|\nabla Q\|_{L^{2}}^{\frac{N \alpha+2 b}{2}}\|Q\|_{L^{2}}^{\frac{4-2 b-(N-2) \alpha}{2}}\right]
$$

where $P(f)$ is as in (1.12). We have the following Pohozaev's identities (see e.g., [20])

$$
\|Q\|_{L^{2}}^{2}=\frac{4-2 b-(N-2) \alpha}{N \alpha+2 b}\|\nabla Q\|_{L^{2}}^{2}=\frac{4-2 b-(N-2) \alpha}{2(\alpha+2)} P(Q) .
$$

In particular, we have

$$
C_{\mathrm{opt}}=\frac{2(\alpha+2)}{N \alpha+2 b}\left(\|\nabla Q\|_{L^{2}}\|Q\|_{L^{2}}^{\sigma_{\mathrm{c}}}\right)^{-\frac{N \alpha-4+2 b}{2}}
$$

We also have

$$
E(Q)=\frac{N \alpha-4+2 b}{2(N \alpha+2 b)}\|\nabla Q\|_{L^{2}}^{2}=\frac{N \alpha-4+2 b}{4(\alpha+2)} P(Q)
$$

hence

$$
E(Q)[M(Q)]^{\sigma_{\mathrm{c}}}=\frac{N \alpha-4+2 b}{2(N \alpha+2 b)}\left(\|\nabla Q\|_{L^{2}}\|Q\|_{L^{2}}^{\sigma_{\mathrm{c}}}\right)^{2}
$$

2.3. Profile decompositions. In this subsection, we recall the linear profile decomposition and construct some nonlinear profiles associated to (1.2). Let us start with the following result due to [23,31] (see also $[21,22])$.

Lemma 2.5 (Linear profile decomposition [21-23,31]). Let $N \geq 1,0<b<\min \{2, N\}$, and $\frac{4-2 b}{N}<\alpha<$ $\alpha(N)$. Let $\left(\phi_{n}\right)_{n \geq 1}$ be a uniformly bounded sequence in $H^{1}$. Then for each integer $J \geq 1$, there exists a subsequence, still denoted by $\phi_{n}$, and

- for each $1 \leq j \leq J$, there exists a fixed profile $\psi^{j} \in H^{1}$;

- for each $1 \leq j \leq J$, there exists a sequence of time shifts $\left(t_{n}^{j}\right)_{n \geq 1} \subset \mathbb{R}$;

- for each $1 \leq j \leq J$, there exists a sequence of space shifts $\left(x_{n}^{j}\right)_{n \geq 1} \subset \mathbb{R}^{N}$;

- there exists a sequence of remainders $\left(W_{n}^{J}\right)_{n \geq 1} \subset H^{1}$;

such that

$$
\phi_{n}(x)=\sum_{j=1}^{J} e^{-i t_{n}^{j} \Delta} \psi^{j}\left(x-x_{n}^{j}\right)+W_{n}^{J}(x) .
$$

The time and space shifts have a pairwise divergence property, i.e., for $1 \leq j \neq k \leq J$, we have

$$
\lim _{n \rightarrow \infty}\left|t_{n}^{j}-t_{n}^{k}\right|+\left|x_{n}^{j}-x_{n}^{k}\right|=\infty
$$

The remainder has the following asymptotic smallness property

$$
\lim _{J \rightarrow \infty}\left[\lim _{n \rightarrow \infty}\left\|e^{i t \Delta} W_{n}^{J}\right\|_{S\left(\dot{H}^{\gamma_{\mathrm{c}}}\right) \cap L_{t}^{\infty}\left(\mathbb{R}, L_{x}^{\frac{2 N}{N-2 \gamma_{\mathrm{c}}}}\right)}\right]=0,
$$


where $\gamma_{\mathrm{c}}$ is as in (1.5). Moreover, for fixed $J$ and $\gamma \in[0,1]$, we have the asymptotic Pythagorean expansions

$$
\left\|\phi_{n}\right\|_{\dot{H}^{\gamma}}^{2}=\sum_{j=1}^{J}\left\|\psi^{j}\right\|_{\dot{H}^{\gamma}}^{2}+\left\|W_{n}^{J}\right\|_{\dot{H}^{\gamma}}^{2}+o_{n}(1) .
$$

Finally, we may assume either $t_{n}^{j} \equiv 0$ or $t_{n}^{j} \rightarrow \pm \infty$, and either $x_{n}^{j} \equiv 0$ or $\left|x_{n}^{j}\right| \rightarrow \infty$.

In the next lemmas, we will construct nonlinear profiles associated to the linear ones with either divergent time or divergent space shifts.

Lemma 2.6 (Nonlinear profile with divergent time shift and no space translation). Let $N \geq 2,0<b<$ $\min \left\{2, \frac{N}{2}\right\}$, and $\frac{4-2 b}{N}<\alpha<\alpha(N)$. Let $\psi \in H^{1}$ and $t_{n} \rightarrow \infty$. Let $v_{n}: C\left(\left(-T_{*}, T^{*}\right), H^{1}\right)$ denote the maximal solution to (1.2) with initial data

$$
v_{n}(0, x)=e^{-i t_{n} \Delta} \psi(x) .
$$

Then for $n$ sufficiently large, $v_{n}$ exists globally backward in time, i.e., $T_{*}=\infty$. Moreover, we have for any $0 \leq T<T^{*}$,

$$
\lim _{n \rightarrow \infty}\left\|\langle\nabla\rangle\left(v_{n}-\psi_{n}\right)\right\|_{S\left((-\infty, T), L^{2}\right)}+\left\|v_{n}-\psi_{n}\right\|_{S\left((-\infty, T), \dot{H}^{\gamma_{c}}\right)}=0
$$

where

$$
\psi_{n}(t, x):=e^{i\left(t-t_{n}\right) \Delta} \psi(x)
$$

In addition, we have

$$
\lim _{n \rightarrow \infty}\left\|v_{n}-\psi_{n}\right\|_{L_{t}^{\infty}\left((-\infty, T), H_{x}^{1}\right)}=0 .
$$

Similarly, if $t_{n} \rightarrow-\infty$ and $v_{n}: C\left(\left(-T_{*}, T^{*}\right), H^{1}\right)$ is the maximal solution to (1.2) with initial data (2.10), then for $n$ sufficiently large, $v_{n}$ exists globally forward in time, i.e., $T^{*}=\infty$. Moreover, we have for any $0 \leq T<T_{*}$,

$$
\lim _{n \rightarrow \infty}\left\|\langle\nabla\rangle\left(v_{n}-\psi_{n}\right)\right\|_{S\left((-T, \infty), L^{2}\right)}+\left\|v_{n}-\psi_{n}\right\|_{S\left((-T, \infty), \dot{H}^{\gamma_{c}}\right)}=0
$$

where $\psi_{n}$ is as in (2.12). Moreover,

$$
\lim _{n \rightarrow \infty}\left\|v_{n}-\psi_{n}\right\|_{L_{t}^{\infty}\left((-T, \infty), H_{x}^{1}\right)}=0
$$

Proof. We only treat the first point, the second point is similar. We see that $\psi_{n}$ satisfies

$$
i \partial_{t} \psi_{n}+\Delta \psi_{n}+|x|^{-b}\left|\psi_{n}\right|^{\alpha} \psi_{n}=e_{n}
$$

with $e_{n}:=|x|^{-b}\left|\psi_{n}\right|^{\alpha} \psi_{n}$. Since $v_{n}(0)=\psi_{n}(0)$, the result follows from the stability given in Lemma 2.4 provided that

$$
\lim _{n \rightarrow \infty}\left\|\langle\nabla\rangle e_{n}\right\|_{S^{\prime}\left((-\infty, T), L^{2}\right)}+\left\|e_{n}\right\|_{S^{\prime}\left((-\infty, T), \dot{H}^{-} \gamma_{c}\right)}=0 .
$$

By Lemma 2.2, we have

$$
\begin{aligned}
\left\|\langle\nabla\rangle e_{n}\right\|_{S^{\prime}\left((-\infty, T), L^{2}\right)} & =\left\|\langle\nabla\rangle\left(|x|^{-b}\left|\psi_{n}\right|^{\alpha} \psi_{n}\right)\right\|_{S^{\prime}\left((-\infty, T), L^{2}\right)} \\
& =\left\|\langle\nabla\rangle\left(|x|^{-b}\left|e^{i t \Delta} \psi\right|^{\alpha} e^{i t \Delta} \psi\right)\right\|_{S^{\prime}\left(\left(-\infty, T-t_{n}\right), L^{2}\right)} \\
& \lesssim\left\|e^{i t \Delta} \psi\right\|_{L_{t}^{\infty}\left(\left(-\infty, T-t_{n}\right), H_{x}^{1}\right)}^{\theta}\left\|e^{i t \Delta} \psi\right\|_{S\left(\left(-\infty, T-t_{n}\right), \dot{H}^{\gamma_{\mathrm{c}}}\right)}^{\alpha-\theta} \\
& \times\left\|\langle\nabla\rangle e^{i t \Delta} \psi\right\|_{S\left(\left(-\infty, T-t_{n}\right), L^{2}\right)} \rightarrow 0
\end{aligned}
$$

as $n \rightarrow \infty$ as $\langle\nabla\rangle e^{i t \Delta} \psi \in S\left(L^{2}\right)$ and $e^{i t \Delta} \psi \in S\left(\dot{H}^{\gamma_{c}}\right)$. Here we do not include the pairs $(\infty, 2)$ and $\left(\infty, \frac{2 N}{N-2 \gamma_{c}}\right)$ into the definitions of $L^{2}$ and $\dot{H}^{\gamma_{c}}$ admissibility, respectively. Similarly, we have

$$
\begin{aligned}
\left\|e_{n}\right\|_{S^{\prime}\left((-\infty, 0), \dot{H}^{\left.-\gamma_{c}\right)}\right.} & =\left\||x|^{-b}\left|e^{i t \Delta} \psi\right|^{\alpha} e^{i t \Delta} \psi\right\|_{S^{\prime}\left(\left(-\infty, T-t_{n}\right), \dot{H}^{-\gamma_{c}}\right)} \\
& \lesssim\left\|e^{i t \Delta} \psi\right\|_{L_{t}^{\infty}\left(\left(-\infty, T-t_{n}\right), H_{x}^{1}\right)}^{\theta}\left\|e^{i t \Delta} \psi\right\|_{S\left(\left(-\infty, T-t_{n}\right), \dot{H}^{\gamma_{c}}\right)}^{\alpha+1-\theta} \rightarrow 0
\end{aligned}
$$

as $n \rightarrow \infty$. This shows (2.14).

We next show (2.13). To see this, we have from (2.11),

$$
\left\|\langle\nabla\rangle \psi_{n}\right\|_{S\left((-\infty, T), L^{2}\right)}=\left\|\langle\nabla\rangle e^{i t \Delta} \psi\right\|_{S\left(\left(-\infty, T-t_{n}\right), L^{2}\right)} \rightarrow 0 \text { as } n \rightarrow \infty,
$$

and similarly for $\left\|\psi_{n}\right\|_{S\left((-\infty, T), \dot{H}^{\left.\gamma_{\mathrm{c}}\right)}\right.}$ that

$$
\lim _{n \rightarrow \infty}\left\|\langle\nabla\rangle v_{n}\right\|_{S\left((-\infty, T), L^{2}\right)}+\left\|v_{n}\right\|_{S\left((-\infty, T), \dot{H}^{\gamma_{c}}\right)}=0
$$


This together with Strichartz estimates, Lemma 2.2, and the fact that $\psi_{n}(t, x)=e^{i t \Delta} v_{n}(0, x)$ imply $\left\|v_{n}\right\|_{L_{t}^{\infty}\left((-\infty, T), H_{x}^{1}\right)} \lesssim 1$. By Lemma 2.2 , we have

$$
\left\|v_{n}-\psi_{n}\right\|_{L_{t}^{\infty}\left((-\infty, T), H_{x}^{1}\right)} \lesssim\left\|v_{n}\right\|_{L_{t}^{\infty}\left((-\infty, T), H_{x}^{1}\right)}^{\theta}\left\|v_{n}\right\|_{S\left((-\infty, T), \dot{H}^{\left.\gamma_{c}\right)}\right.}^{\alpha-\theta}\left\|\langle\nabla\rangle v_{n}\right\|_{S\left((-\infty, T), L^{2}\right)} \rightarrow 0
$$

as $n \rightarrow \infty$. The proof is complete.

Lemma 2.7 (Nonlinear profile with divergent space shift). Let $N \geq 2,0<b<\min \left\{2, \frac{N}{2}\right\}$, and $\frac{4-2 b}{N}<$ $\alpha<\alpha(N)$. Let $\psi \in H^{1}$ and $\left(t_{n}, x_{n}\right) \in \mathbb{R} \times \mathbb{R}^{N}$ satisfying $\left|x_{n}\right| \rightarrow \infty$ as $n \rightarrow \infty$. Let $v_{n}: C\left(\left(-T_{*}, T^{*}\right), H^{1}\right)$ denote the maximal solution to (1.2) with initial data

$$
v_{n}(0, x)=e^{-i t_{n} \Delta} \psi\left(x-x_{n}\right)
$$

Then for $n$ sufficiently large, $v_{n}$ exists globally in time, i.e., $T_{*}=T^{*}=\infty$. Moreover, we have

$$
\lim _{n \rightarrow \infty}\left\|\langle\nabla\rangle\left(v_{n}-\psi_{n}\right)\right\|_{S\left(L^{2}\right)}+\left\|v_{n}-\psi_{n}\right\|_{S\left(\dot{H}^{\gamma_{c}}\right)}=0
$$

where

$$
\psi_{n}(t, x):=e^{i\left(t-t_{n}\right) \Delta} \psi\left(x-x_{n}\right)
$$

Remark 2.2. The construction of nonlinear profiles with divergent space translations was first established by Miao, Murphy, and Zheng [41] for (1.2) with $\alpha=2$ and $N=3$. This result was recently extended to (1.2) with $N \geq 2$ by Cardoso, Farah, Guzmán, and Murphy [6]. Here we give a refine result with a simple proof compared to the ones in [6,41]. More precisely, for a linear profile with a divergent space shift, the associated nonlinear profile is close to the solution of the underlying linear Schrödinger equation.

Proof of Lemma 2.7. As in the proof of Lemma 2.6, it suffices to show

$$
\lim _{n \rightarrow \infty}\left\|\langle\nabla\rangle e_{n}\right\|_{S^{\prime}\left(L^{2}\right)}+\left\|e_{n}\right\|_{S^{\prime}\left(\dot{H}^{-}-\gamma_{\mathrm{c}}\right)}=0 .
$$

To see this, we take $\varepsilon>0$. We have

$$
\begin{aligned}
\left\|\langle\nabla\rangle e_{n}\right\|_{S^{\prime}\left(L^{2}\right)}= & \left\|\langle\nabla\rangle\left(|x|^{-b}\left|\psi_{n}\right|^{\alpha} \psi_{n}\right)\right\|_{S^{\prime}\left(L^{2}\right)} \\
= & \left\|\langle\nabla\rangle\left(\left|x+x_{n}\right|^{-b}\left|e^{i t \Delta} \psi\right|^{\alpha} e^{i t \Delta} \psi\right)\right\|_{S^{\prime}\left(L^{2}\right)} \\
\leq & \left\|\langle\nabla\rangle\left(\left|x+x_{n}\right|^{-b}\left|e^{i t \Delta} \psi\right|^{\alpha} e^{i t \Delta} \psi\right)\right\|_{S^{\prime}\left(L^{2}\left(B_{R}\right)\right)} \\
& +\left\|\langle\nabla\rangle\left(\left|x+x_{n}\right|^{-b}\left|e^{i t \Delta} \psi\right|^{\alpha} e^{i t \Delta} \psi\right)\right\|_{S^{\prime}\left(L^{2}\left(B_{R}^{c}\right)\right)}
\end{aligned}
$$

where $B_{R}:=\left\{x \in \mathbb{R}^{N}:|x| \leq R\right\}$ and $B_{R}^{c}=\mathbb{R}^{N} \backslash B_{R}$ with $R>0$ to be chosen later.

On $B_{R}^{c}$, by splitting $B_{R}^{c}=\Omega_{1} \cup \Omega_{2}$ with

$$
\Omega_{1}=\left\{x \in \mathbb{R}^{N}:|x| \geq R,\left|x+x_{n}\right| \leq 1\right\}, \quad \Omega_{2}=\left\{x \in \mathbb{R}^{N}:|x| \geq R,\left|x+x_{n}\right| \geq 1\right\},
$$

the same argument as in the proof of Lemma 2.2 implies that

$$
\left\|\langle\nabla\rangle\left(\left|x+x_{n}\right|^{-b}|\varphi|^{\alpha} \varphi\right)\right\|_{S^{\prime}\left(L^{2}\left(B_{R}^{c}\right)\right)} \lesssim\|\varphi\|_{L_{t}^{\infty} H_{x}^{1}\left(B_{R}^{c}\right)}^{\theta}\|\varphi\|_{S\left(\dot{\left.H^{\gamma_{c}}\left(B_{R}^{c}\right)\right)}\right.}^{\alpha-\theta}\|\langle\nabla\rangle \varphi\|_{S\left(L^{2}\left(B_{R}^{c}\right)\right)},
$$

where

$$
\varphi(t, x):=e^{i t \Delta} \psi(x)
$$

As $\varphi \in S\left(\dot{H}^{\gamma_{c}}\right)$ and $\langle\nabla\rangle \varphi \in S\left(L^{2}\right)$, we see that

$$
\|\varphi\|_{S\left(\dot{H}^{\gamma_{c}}\left(B_{R}^{c}\right)\right)},\|\langle\nabla\rangle \varphi\|_{S\left(L^{2}\left(B_{R}^{c}\right)\right)} \rightarrow 0 \text { as } R \rightarrow \infty .
$$

Note that it is crucial to exclude the pairs $(\infty, 2)$ and $\left(\infty, \frac{2 N}{N-2 \gamma_{c}}\right)$ from the definitions of $L^{2}$ and $\dot{H}^{\gamma_{c}}$ admissible conditions, respectively. This shows that for $R_{0}>0$ sufficiently large,

$$
\left\|\langle\nabla\rangle\left(\left|x+x_{n}\right|^{-b}|\varphi|^{\alpha} \varphi\right)\right\|_{S^{\prime}\left(L^{2}\left(B_{R_{0}}^{c}\right)\right)}<\frac{\varepsilon}{4}
$$

for all $n \geq 1$.

Next, for $x \in B_{R_{0}}$, as $\left|x_{n}\right| \rightarrow \infty$, we have $\left|x+x_{n}\right| \geq\left|x_{n}\right|-|x| \geq \frac{\left|x_{n}\right|}{2}$ for $n$ sufficiently large. It follows from Lemma 2.2 that

$$
\left\|\left|x+x_{n}\right|^{-b}|\varphi|^{\alpha} \varphi\right\|_{S^{\prime}\left(L^{2}\left(B_{R_{0}}\right)\right)} \lesssim\left|x_{n}\right|^{-b}\left\||\varphi|^{\alpha} \varphi\right\|_{S^{\prime}\left(L^{2}\right)} \lesssim\left|x_{n}\right|^{-b}\|\varphi\|_{S\left(\dot{H}^{\left.\gamma_{c}\right)}\right)}^{\alpha}\|\varphi\|_{S\left(L^{2}\right)} \rightarrow 0
$$


as $n \rightarrow \infty$. Similarly, we have

$$
\begin{aligned}
\| \nabla\left(\left|x+x_{n}\right|^{-b}|\varphi|^{\alpha} \varphi\right) & \|_{S^{\prime}\left(L^{2}\left(B_{R_{0}}\right)\right)} \\
& \lesssim\left\|\left|x+x_{n}\right|^{-b} \nabla\left(|\varphi|^{\alpha} \varphi\right)\right\|_{S^{\prime}\left(L^{2}\left(B_{R_{0}}\right)\right)}+\left\|\left|x+x_{n}\right|^{-b-1}|\varphi|^{\alpha} \varphi\right\|_{S^{\prime}\left(L^{2}\left(B_{R_{0}}\right)\right)} \\
& \lesssim\left|x_{n}\right|^{-b}\left\|\nabla\left(|\varphi|^{\alpha} \varphi\right)\right\|_{S^{\prime}\left(L^{2}\right)}+\left|x_{n}\right|^{-b-1}\left\||\varphi|^{\alpha} \varphi\right\|_{S^{\prime}\left(L^{2}\right)} \\
& \lesssim\left|x_{n}\right|^{-b}\|\varphi\|_{S\left(\dot{H}^{\left.\gamma_{c}\right)}\right.}^{\alpha}\|\nabla \varphi\|_{S\left(L^{2}\right)}+\left|x_{n}\right|^{-b-1}\|\varphi\|_{S\left(\dot{H}^{\left.\gamma_{c}\right)}\right.}^{\alpha}\|\varphi\|_{S\left(L^{2}\right)} \rightarrow 0
\end{aligned}
$$

as $n \rightarrow \infty$. Thus there exists $n_{1}>0$ sufficiently large such that for all $n \geq n_{1}$,

$$
\left\|\langle\nabla\rangle\left(\left|x+x_{n}\right|^{-b}|\varphi|^{\alpha} \varphi\right)\right\|_{S^{\prime}\left(L^{2}\left(B_{R_{0}}\right)\right)}<\frac{\varepsilon}{4},
$$

hence

$$
\left\|\langle\nabla\rangle\left(\left|x+x_{n}\right|^{-b}|\varphi|^{\alpha} \varphi\right)\right\|_{S^{\prime}\left(L^{2}\right)}<\frac{\varepsilon}{2} .
$$

A similar argument show that for all $n \geq n_{2}$ with $n_{2}>0$ sufficiently large,

$$
\left\|\left|x+x_{n}\right|^{-b}|\varphi|^{\alpha} \varphi\right\|_{S^{\prime}\left(\dot{H}^{\left.-\gamma_{c}\right)}\right.}<\frac{\varepsilon}{2} .
$$

Therefore, we have for all $n \geq \max \left\{n_{1}, n_{2}\right\}$,

$$
\left\|\langle\nabla\rangle\left(\left|x+x_{n}\right|^{-b}|\varphi|^{\alpha} \varphi\right)\right\|_{S^{\prime}\left(L^{2}\right)}+\left\|\left|x+x_{n}\right|^{-b}|\varphi|^{\alpha} \varphi\right\|_{S^{\prime}\left(\dot{H}^{-} \gamma_{\mathrm{c}}\right)}<\varepsilon
$$

which proves (2.17). The proof is complete.

2.4. Energy scattering. In this section, we give the proof of the scattering criterion given in Theorem 1.1. To this end, we need the following coercivity lemma.

Lemma 2.8. Let $N \geq 1,0<b<\min \{2, N\}$, and $\frac{4-2 b}{N}<\alpha<\alpha(N)$. Let $f \in H^{1}$ satisfy

$$
P(f)[M(f)]^{\sigma_{\mathrm{c}}} \leq A<P(Q)[M(Q)]^{\sigma_{\mathrm{c}}}
$$

for some constant $A>0$. Then there exists $\nu=\nu(A, Q)>0$ such that

$$
\begin{aligned}
& G(f) \geq \nu\|\nabla f\|_{L^{2}}^{2}, \\
& E(f) \geq \frac{\nu}{2}\|\nabla f\|_{L^{2}}^{2} .
\end{aligned}
$$

Proof. We write

$$
A=(1-\rho) P(Q)[M(Q)]^{\sigma_{\mathrm{c}}}
$$

for some $\rho=\rho(A, Q) \in(0,1)$. It follows from (2.3), (2.4), (2.5), and (2.18) that

$$
\begin{aligned}
{[P(f)]^{\frac{N \alpha+2 b}{4}} } & \leq C_{\mathrm{opt}}\left(P(f)[M(f)]^{\sigma_{\mathrm{c}}}\right)^{\frac{N \alpha-4+2 b}{4}}\|\nabla f\|_{L^{2}}^{\frac{N \alpha+2 b}{2}} \\
& =\frac{2(\alpha+2)}{N \alpha+2 b}\left(\frac{P(f)[M(f)]^{\sigma_{\mathrm{c}}}}{\|\nabla Q\|_{L^{2}}^{2}\|Q\|_{L^{2}}^{2 \sigma_{\mathrm{c}}}}\right)^{\frac{N \alpha-4+2 b}{4}}\|\nabla f\|_{L^{2}}^{\frac{N \alpha+2 b}{2}} \\
& =\left(\frac{P(f)[M(f)]^{\sigma_{\mathrm{c}}}}{P(Q)[M(Q)]_{L_{\mathrm{c}}^{2}}^{\sigma_{c}}}\right)^{\frac{N \alpha-4+2 b}{4}}\left(\frac{2(\alpha+2)}{N \alpha+2 b}\|\nabla f\|_{L^{2}}^{2}\right)^{\frac{N \alpha+2 b}{4}} \\
& \leq(1-\rho)^{\frac{N \alpha-4+2 b}{4}}\left(\frac{2(\alpha+2)}{N \alpha+2 b}\|\nabla f\|_{L^{2}}^{2}\right)^{\frac{N \alpha+2 b}{4}}
\end{aligned}
$$

which implies

$$
P(f) \leq \frac{2(\alpha+2)}{N \alpha+2 b}(1-\rho)^{\frac{N \alpha-4+2 b}{N \alpha+2 b}}\|\nabla f\|_{L^{2}}^{2}
$$

Thus we get

$$
G(f)=\|\nabla f\|_{L^{2}}^{2}-\frac{N \alpha+2 b}{2(\alpha+2)} P(f) \geq\left(1-(1-\rho)^{\frac{N \alpha-4+2 b}{N \alpha+2 b}}\right)\|\nabla f\|_{L^{2}}^{2}
$$

which proves (2.19). As $N \alpha-4+2 b>0$, we have

$$
E(f)=\frac{1}{2} G(f)+\frac{N \alpha-4+2 b}{2(\alpha+2)} P(f) \geq \frac{1}{2} G(f)
$$

which shows (2.20). The proof is complete. 
We are now able to give the proof of Theorem 1.1.

Proof of Theorem 1.1. Let $u:\left[0, T^{*}\right) \times \mathbb{R}^{N} \rightarrow \mathbb{C}$ be a $H^{1}$-solution to (1.2) satisfying (1.11). By the conservation of mass and energy, we infer from (1.11) that

$$
\sup _{t \in\left[0, T^{*}\right)}\|\nabla u(t)\|_{L^{2}} \leq C(E, Q)<\infty .
$$

By the local well-posedness given in Lemma 2.3, we have $T^{*}=\infty$.

Let $A>0$ and $\delta>0$. We define

$$
S(A, \delta):=\sup \left\{\|u\|_{S\left([0, \infty), \dot{H}^{\left.\gamma_{c}\right)}\right.}: u \text { is a solution to (1.2) satisfying }(2.21)\right\},
$$

where

$$
\sup _{t \in[0, \infty)} P(u(t))[M(u(t))]^{\sigma_{c}} \leq A, \quad E(u)[M(u)]^{\sigma_{c}} \leq \delta .
$$

Thanks to the scattering condition (see again Lemma 2.3) and the definition of $S(A, \delta)$, Theorem 1.1 is reduced to show the following proposition.

Proposition 2.9. Let $N \geq 2,0<b<\min \left\{2, \frac{N}{2}\right\}$, and $\frac{4-2 b}{N}<\alpha<\alpha(N)$. If $A<P(Q)[M(Q)]^{\sigma_{c}}$, then for all ${ }^{1} \delta>0, S(A, \delta)<\infty$.

The proof of Proposition 2.9 is based on the concentration/compactness and rigidity argument introduced by Kenig and Merle [36] (see also [17]). The main difficulty comes from the fact that the potential energy $P(u(t))$ is not conserved along the time evolution of (1.2). To overcome the difficulty, we establish a Pythagorean decomposition along the bounded INLS flow (see Lemma 2.10). In the context of the standard NLS, a similar result was shown by Guevara in [31, Lemma 3.9].

The proof of Proposition 2.9 is done by several steps.

Step 1. Small data scattering. By (2.20), we have

$$
\left\|u_{0}\right\|_{H^{\gamma_{c}}}^{\frac{2}{\gamma_{c}}} \leq\left\|\nabla u_{0}\right\|_{L^{2}}^{2}\left\|u_{0}\right\|_{L^{2}}^{2 \sigma_{c}} \leq \frac{2}{\nu} E\left(u_{0}\right)\left[M\left(u_{0}\right)\right]^{\sigma_{c}} \leq \frac{2 \delta}{\nu} .
$$

By taking $\delta>0$ sufficiently small, we see that $\left\|u_{0}\right\|_{\dot{H}^{\gamma_{c}}}$ is small which, by the small data scattering given in Lemma 2.3, implies $S(A, \delta)<\infty$.

Step 2. Existence of a critical solution. Assume by contradiction that $S(A, \delta)=\infty$ for some $\delta>0$. By Step 1,

$$
\delta_{\mathrm{c}}:=\delta_{\mathrm{c}}(A):=\inf \{\delta>0: S(A, \delta)=\infty\}
$$

is well-defined and positive. From the definition of $\delta_{\mathrm{c}}$, we have the following observations:

(1) If $u$ is a solution to (1.2) satisfying

$$
\sup _{t \in[0, \infty)} P(u(t))[M(u(t))]^{\sigma_{\mathrm{c}}} \leq A, \quad E(u)[M(u)]^{\sigma_{\mathrm{c}}}<\delta_{\mathrm{c}},
$$

then $\|u\|_{S\left([0, \infty), \dot{H}^{\left.\sigma_{c}\right)}\right.}<\infty$ and the solution scatters in $H^{1}$ forward in time.

(2) There exists a sequence of solution $u_{n}$ to (1.2) with initial data $u_{n, 0}$ such that

$$
\begin{aligned}
\sup _{t \in[0, \infty)} P\left(u_{n}(t)\right)\left[M\left(u_{n}(t)\right)\right]^{\sigma_{c}} & \leq A \text { for all } n, \\
E\left(u_{n}\right)\left[M\left(u_{n}\right)\right]^{\sigma_{c}} & \searrow \delta_{\mathrm{c}} \text { as } n \rightarrow \infty, \\
\left\|u_{n}\right\|_{S\left([0, \infty), \dot{H} \gamma_{c}\right)} & =\infty \text { for all } n .
\end{aligned}
$$

We will prove that there exists a critical solution $u_{\mathrm{c}}$ to (1.2) with initial data $u_{\mathrm{c}, 0}$ satisfying

$$
\begin{aligned}
M\left(u_{\mathrm{c}}\right) & =1, \\
\sup _{t \in[0, \infty)} P\left(u_{\mathrm{c}}(t)\right) & \leq A, \\
E\left(u_{\mathrm{c}}\right) & =\delta_{\mathrm{c}}, \\
\left\|u_{\mathrm{c}}\right\|_{S\left([0, \infty), \dot{H}^{\left.\gamma_{\mathrm{c}}\right)}\right.} & =\infty .
\end{aligned}
$$

\footnotetext{
${ }^{1}$ Note the energy is positive due to Lemma 2.8 .
} 
To see this, we consider the sequence $\left(u_{n, 0}\right)_{n \geq 1}$. Thanks to the scaling (1.4), we can assume that $M\left(u_{n, 0}\right)=1$ for all $n$. By the conservation of mass and energy, (2.23) becomes

$$
\begin{aligned}
M\left(u_{n, 0}\right) & =1 \text { for all } n, \\
\sup _{t \in[0, \infty)} P\left(u_{n}(t)\right) & \leq A \text { for all } n, \\
E\left(u_{n, 0}\right) & \searrow \delta_{c} \text { as } n \rightarrow \infty, \\
\left\|u_{n}\right\|_{S\left([0, \infty), \dot{H}^{\gamma_{c}}\right)} & =\infty \text { for all } n .
\end{aligned}
$$

Since $\left(u_{n, 0}\right)_{n \geq 1}$ is bounded in $H^{1}$, we apply the linear profile decomposition to $u_{n, 0}$ and get

$$
u_{n, 0}(x)=\sum_{j=1}^{J} e^{-i t_{n}^{j} \Delta} \psi^{j}\left(x-x_{n}^{j}\right)+W_{n}^{J}(x)
$$

with the following properties:

$$
\begin{aligned}
& 1 \leq j \neq k \leq J, \quad \lim _{n \rightarrow \infty}\left|t_{n}^{j}-t_{n}^{k}\right|+\left|x_{n}^{j}-x_{n}^{k}\right|=\infty, \\
& \lim _{J \rightarrow \infty}\left[\lim _{n \rightarrow \infty}\left\|e^{i t \Delta} W_{n}^{J}\right\|_{S\left(\dot{H}^{\gamma_{\mathrm{c}}}\right) \cap L_{t}^{\infty}\left(\mathbb{R}, L_{x}^{N-2 \gamma_{\mathrm{c}}}\right)}\right]=0,
\end{aligned}
$$

and for fixed $J$ and $\gamma \in[0,1]$,

$$
\left\|u_{n, 0}\right\|_{\dot{H}^{\gamma}}^{2}=\sum_{j=1}^{J}\left\|\psi^{j}\right\|_{\dot{H}^{\gamma}}^{2}+\left\|W_{n}^{J}\right\|_{\dot{H}^{\gamma}}^{2}+o_{n}(1) .
$$

Moreover, we also have the following Pythagorean expansions of the potential and total energies:

$$
\begin{aligned}
& P\left(u_{n, 0}\right)=\sum_{j=1}^{J} P\left(e^{-i t_{n}^{j} \Delta} \psi^{j}\left(\cdot-x_{n}^{j}\right)\right)+P\left(W_{n}^{J}\right)+o_{n}(1), \\
& E\left(u_{n, 0}\right)=\sum_{j=1}^{J} E\left(e^{-i t_{n}^{j} \Delta} \psi^{j}\left(\cdot-x_{n}^{j}\right)\right)+E\left(W_{n}^{J}\right)+o_{n}(1) .
\end{aligned}
$$

For the proof of the above expansions, we refer to [21] (see also [22]). We now define the nonlinear profiles $v^{j}: I^{j} \times \mathbb{R}^{N} \rightarrow \mathbb{C}$ associated to $\psi^{j}, t_{n}^{j}$, and $x_{n}^{j}$ as follows:

- If $x_{n}^{j} \equiv 0$ and $t_{n}^{j} \equiv 0$, then $v^{j}$ is the maximal lifespan solution to (1.2) with initial data $\left.v^{j}\right|_{t=0}=\psi^{j}$.

- If $x_{n}^{j} \equiv 0$ and $t_{n}^{j} \rightarrow-\infty$, then $v^{j}$ is the maximal lifespan solution to (1.2) that scatters to $e^{i t \Delta} \psi^{j}$ as $t \rightarrow \infty$ (Such a solution exists due to Lemma 2.6). In particular, $\left\|v^{j}\right\|_{S\left((0, \infty), \dot{H}^{\left.\gamma_{c}\right)}\right.}<\infty$ and $\left\|v^{j}\left(-t_{n}^{j}\right)-e^{-i t_{n}^{j} \Delta} \psi^{j}\right\|_{H^{1}} \rightarrow 0$ as $n \rightarrow \infty$.

- If $x_{n}^{j} \equiv 0$ and $t_{n}^{j} \rightarrow \infty$, then $v^{j}$ is the maximal lifespan solution to (1.2) that scatters to $e^{i t \Delta} \psi^{j}$ as $t \rightarrow-\infty$. In particular, $\left\|v^{j}\right\|_{S\left((-\infty, 0), \dot{H}^{\gamma_{c}}\right)}<\infty$ and $\left\|v^{j}\left(-t_{n}^{j}\right)-e^{-i t_{n}^{j} \Delta} \psi^{j}\right\|_{H^{1}} \rightarrow 0$ as $n \rightarrow \infty$.

- If $\left|x_{n}^{j}\right| \rightarrow \infty$, then we simply take $v^{j}(t)=e^{i t \Delta} \psi^{j}$.

For each $j, n \geq 1$, we introduce $v_{n}^{j}: I_{n}^{j} \times \mathbb{R}^{N} \rightarrow \mathbb{C}$ defined by

- if $x_{n}^{j} \equiv 0$, then $v_{n}^{j}(t):=v^{j}\left(t-t_{n}^{j}\right)$, where $I_{n}^{j}:=\left\{t \in \mathbb{R}: t-t_{n}^{j} \in I^{j}\right\}$.

- if $\left|x_{n}^{j}\right| \rightarrow \infty$, we define $v_{n}^{j}$ a solution to (1.2) with initial data $v_{n}^{j}(0, x)=v^{j}\left(-t_{n}^{j}, x-x_{n}^{j}\right)=e^{-i t_{n}^{j} \Delta} \psi^{j}(x-$ $\left.x_{n}^{j}\right)$. It follows from Lemma 2.7 that for $n$ sufficiently large, $v_{n}^{j}$ exists globally in time and scatters in $H^{1}$ in both directions.

We have from the definition of $v_{n}^{j}$ and the continuity of the linear flow that

$$
\left\|v_{n}^{j}(0)-e^{-i t_{n}^{j} \Delta} \psi^{j}\left(\cdot-x_{n}^{j}\right)\right\|_{H^{1}} \rightarrow 0 \quad \text { as } n \rightarrow \infty .
$$

Thus we rewrite $(2.26)$ as

$$
u_{n, 0}(x)=\sum_{j=1}^{J} v_{n}^{j}(0, x)+\tilde{W}_{n}^{J}(x)
$$

where

$$
\tilde{W}_{n}^{J}(x)=\sum_{j=1}^{J} e^{-i t_{n}^{j} \Delta} \psi^{j}\left(x-x_{n}^{j}\right)-v_{n}^{j}(0, x)+W_{n}^{J}(x) .
$$


By Strichartz estimates, we have

$$
\left\|e^{i t \Delta} \tilde{W}_{n}^{J}\right\|_{S\left(\dot{H}^{\gamma_{c}}\right) \cap L_{t}^{\infty}\left(\mathbb{R}, L_{x}^{\frac{2 N}{N-2 \gamma_{\mathrm{c}}}}\right)} \lesssim \sum_{j=1}^{J}\left\|e^{-i t_{n}^{j} \Delta} \psi^{j}\left(\cdot-x_{n}^{j}\right)-v_{n}^{j}(0)\right\|_{H^{1}}+\left\|e^{i t \Delta} W_{n}^{J}\right\|_{S\left(\dot{H}^{\gamma_{\mathrm{c}}}\right) \cap L_{t}^{\infty}\left(\mathbb{R}, L_{x}^{\frac{2 N}{N-2 \gamma_{\mathrm{c}}}}\right)}
$$

which, by (2.28) and (2.32), implies that

Using the fact that

$$
\lim _{J \rightarrow \infty}\left[\lim _{n \rightarrow \infty}\left\|e^{i t \Delta} \tilde{W}_{n}^{J}\right\|_{S\left(\dot{H} \gamma_{c}\right) \cap L_{t}^{\infty}\left(\mathbb{R}, L_{x}^{\frac{2 N}{N-2 \gamma_{c}}}\right)}\right]=0 .
$$

and (see [22, Lemma 4.3])

$$
\left|\|\nabla f\|_{L^{2}}^{2}-\|\nabla g\|_{L^{2}}^{2}\right| \lesssim\|\nabla f-\nabla g\|_{L^{2}}\left(\|\nabla f\|_{L^{2}}+\|\nabla g\|_{L^{2}}\right)
$$

$$
|P(f)-P(g)| \lesssim\|f-g\|_{L^{\alpha+2}}\left(\|f\|_{L^{\alpha+2}}^{\alpha+1}+\|g\|_{L^{\alpha+2}}^{\alpha+1}\right)+\|f-g\|_{L^{r}}\left(\|f\|_{L^{r}}^{\alpha+1}+\|g\|_{L^{r}}^{\alpha+1}\right)
$$

for some $\frac{2 N \alpha}{N-b}<r<2^{*}$, where

$$
2^{*}:=\left\{\begin{array}{cll}
\frac{2 N}{N-2} & \text { if } \quad N \geq 3 \\
\infty & \text { if } \quad N=1,2
\end{array}\right.
$$

we infer from (2.31), Sobolev embedding, and (2.32) that

$$
E\left(u_{n, 0}\right)=\sum_{j=1}^{J} E\left(v_{n}^{j}(0)\right)+E\left(\tilde{W}_{n}^{J}\right)+o_{n}(1) .
$$

Next, we show the following Pythagorean expansion along the bounded INLS flow (see [31, Lemma 3.9] for a similar result in the context of NLS).

Lemma 2.10 (Pythagorean expansion along the bounded INLS flow). Let $T \in(0, \infty)$ be a fixed time. Assume that for all $n \geq 1, u_{n}(t):=\operatorname{INLS}(t) u_{n, 0}$ exists up to time $T$ and satisfies

$$
\lim _{n \rightarrow \infty} \sup _{t \in[0, T]}\left\|\nabla u_{n}(t)\right\|_{L^{2}}<\infty
$$

where $\operatorname{INLS}(t) f$ denotes the solution to (1.2) with initial data $f$ at time $t=0$. We consider the nonlinear profile (2.33). Denote $\tilde{W}_{n}^{J}(t):=\operatorname{INLS}(t) \tilde{W}_{n}^{J}$. Then for all $t \in[0, T]$,

$$
\left\|\nabla u_{n}(t)\right\|_{L^{2}}^{2}=\sum_{j=1}^{J}\left\|\nabla v_{n}^{j}(t)\right\|_{L^{2}}^{2}+\left\|\nabla \tilde{W}_{n}^{J}(t)\right\|_{L^{2}}^{2}+o_{J, n}(1),
$$

where $o_{J, n}(1) \rightarrow 0$ as $J, n \rightarrow \infty$ uniformly on $0 \leq t \leq T$. In particular, we have for all $t \in[0, T]$,

$$
P\left(u_{n}(t)\right)=\sum_{j=1}^{J} P\left(v_{n}^{j}(t)\right)+P\left(\tilde{W}_{n}^{J}(t)\right)+o_{J, n}(1) .
$$

Proof. By (2.29), there exists $J_{0}$ large enough such that $\left\|\psi^{j}\right\|_{H^{1}}$ sufficiently small for all $j \geq J_{0}+1$. By the triangle inequality using (2.32), we see that for $n$ large, $\left\|v_{n}^{j}(0)\right\|_{H^{1}}$ is small which, by the small data theory, implies that $v_{n}^{j}$ exists globally in time and scatters in $H^{1}$ in both directions. Moreover, we can assume that for all $1 \leq j \leq J_{0}, x_{n}^{j} \equiv 0$ since otherwise, if $\left|x_{n}^{j}\right| \rightarrow \infty$, then by Lemma 2.7, we have for $n$ large, $v_{n}^{j}$ exists globally in time and scatters in $H^{1}$ in both directions. In particular, we have for all $j \geq J_{0}+1$

$$
\left\|v_{n}^{j}\right\|_{S\left(\dot{H}^{\gamma_{\mathrm{c}}}\right)}<\infty
$$

for $n$ large. We reorder the first $J_{0}$ profiles and let $0 \leq J_{2} \leq J_{0}$ such that

- for any $1 \leq j \leq J_{2}$, the time shifts $t_{n}^{j} \equiv 0$ for all $n$. Here $J_{2} \equiv 0$ means that there is no $j$ in this case. Note that by the pairwise divergence property (2.9), we have $J_{2} \leq 1$.

- for any $J_{2}+1 \leq j \leq J_{0}$, the time shifts $\left|t_{n}^{j}\right| \rightarrow \infty$ as $n \rightarrow \infty$. Here $J_{2}=J_{0}$ means that there is no $j$ in this case.

In the following, we only consider the case $J_{2}=1$. The one for $J_{2}=0$ is treated similarly (even simpler). Fix $T \in(0, \infty)$ and assume that $u_{n}(t)=\operatorname{INLS}(t) u_{n, 0}$ exists up to time $T$ and satisfies (2.38). We observe that for $2 \leq j \leq J_{0}$,

$$
\left\|v_{n}^{j}\right\|_{S\left([0, T], \dot{H}^{\gamma_{c}}\right)} \rightarrow 0 \text { as } n \rightarrow \infty
$$

Indeed, if $t_{n}^{j} \rightarrow \infty$, then as $\left\|v^{j}\right\|_{S\left((-\infty, 0), \dot{H}^{\gamma_{c}}\right)}<\infty$, we have

$$
\left\|v_{n}^{j}\right\|_{S\left([0, T], \dot{H}^{\gamma_{c}}\right)}=\left\|v^{j}\right\|_{S\left(\left[-t_{n}^{j}, T-t_{n}^{j}\right], \dot{H}^{\gamma_{\mathrm{c}}}\right)} \rightarrow 0
$$




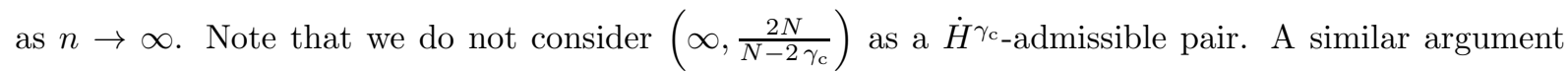
goes for $t_{n}^{j} \rightarrow-\infty$.

Moreover, for $2 \leq j \leq J_{0}$, we have for all $2<r \leq 2^{*}$,

$$
\left\|v_{n}^{j}\right\|_{L_{t}^{\infty}\left([0, T], L_{x}^{r}\right)} \rightarrow 0 \text { as } n \rightarrow \infty .
$$

In fact, we have

$$
\begin{aligned}
\left\|v_{n}^{j}\right\|_{L_{t}^{\infty}\left([0, T], L_{x}^{r}\right)} & \leq\left\|e^{i\left(t-t_{n}^{j}\right) \Delta} \psi^{j}\left(\cdot-x_{n}^{j}\right)\right\|_{L_{t}^{\infty}\left([0, T], L_{x}^{r}\right)}+\left\|v_{n}^{j}-e^{i\left(t-t_{n}^{j}\right) \Delta} \psi^{j}\left(\cdot-x_{n}^{j}\right)\right\|_{L_{t}^{\infty}\left([0, T], L_{x}^{r}\right)} \\
& \leq\left\|e^{i\left(t-t_{n}^{j}\right) \Delta} \psi^{j}\right\|_{L_{t}^{\infty}\left([0, T], L_{x}^{r}\right)}+C\left\|v_{n}^{j}-e^{i\left(t-t_{n}^{j}\right) \Delta} \psi^{j}\left(\cdot-x_{n}^{j}\right)\right\|_{L_{t}^{\infty}\left([0, T], H_{x}^{1}\right)} .
\end{aligned}
$$

By the decay of the linear flow, the first term tends to zero as $n$ tends to infinity due to $\left|t_{n}^{j}\right| \rightarrow \infty$. For the second term, we use the Duhamel formula

$$
v_{n}^{j}(t)=e^{i t \Delta} v_{n}^{j}(0)+i \int_{0}^{t} e^{i(t-s) \Delta}|x|^{-b}\left|v_{n}^{j}(s)\right|^{\alpha} v_{n}^{j}(s) d s,
$$

Strichartz estimates, and Lemma 2.2 to have

$$
\begin{aligned}
\left\|v_{n}^{j}\right\|_{L_{t}^{\infty}\left([0, T], H_{x}^{1}\right)} & +\left\|\langle\nabla\rangle v_{n}^{j}\right\|_{S\left([0, T], L^{2}\right)} \\
& \lesssim\left\|v_{n}^{j}(0)\right\|_{H^{1}}+\left\|v_{n}^{j}\right\|_{L_{t}^{\infty}\left([0, T], H_{x}^{1}\right)}^{\theta}\left\|v_{n}^{j}\right\|_{S\left([0, T], \dot{H}^{\gamma_{c}}\right)}^{\alpha-\theta}\left\|\langle\nabla\rangle v_{n}^{j}\right\|_{S\left([0, T], L^{2}\right)} \\
& \lesssim\left\|e^{-i t_{n}^{j} \Delta} \psi^{j}\right\|_{H^{1}}+1+\left(\left\|v_{n}^{j}\right\|_{L_{t}^{\infty}\left([0, T], H_{x}^{1}\right)}+\left\|\langle\nabla\rangle v_{n}^{j}\right\|_{S\left([0, T], L^{2}\right)}\right)^{1+\theta}\left\|v_{n}^{j}\right\|_{S\left([0, T], \dot{H}^{\left.\gamma_{c}\right)}\right.}^{\alpha-\theta}
\end{aligned}
$$

It follows from (2.42) that

$$
\left\|v_{n}^{j}\right\|_{L_{t}^{\infty}\left([0, T], H_{x}^{1}\right)}+\left\|\langle\nabla\rangle v_{n}^{j}\right\|_{S\left([0, T], L^{2}\right)} \lesssim 1
$$

Similarly, we have

$$
\begin{aligned}
\| v_{n}^{j}- & e^{i\left(t-t_{n}^{j}\right) \Delta} \psi^{j}\left(\cdot-x_{n}^{j}\right) \|_{L_{t}^{\infty}\left([0, T], H_{x}^{1}\right)} \\
\lesssim & \left\|e^{i t \Delta} v_{n}^{j}(0)-e^{i\left(t-t_{n}^{j}\right) \Delta} \psi^{j}\left(\cdot-x_{n}^{j}\right)\right\|_{L_{t}^{\infty}\left([0, T], H_{x}^{1}\right)} \\
& \quad+\left\|v_{n}^{j}\right\|_{L_{t}^{\infty}\left([0, T], H_{x}^{1}\right)}^{\theta}\left\|v_{n}^{j}\right\|_{S\left([0, T], \dot{H}^{\gamma_{c}}\right)}^{\alpha-\theta}\left\|\langle\nabla\rangle v_{n}^{j}\right\|_{S\left([0, T], L^{2}\right)} \\
& \lesssim\left\|v_{n}^{j}(0)-e^{-i t_{n}^{j} \Delta} \psi^{j}\left(\cdot-x_{n}^{j}\right)\right\|_{H^{1}}+\left\|v_{n}^{j}\right\|_{L_{t}^{\infty}\left([0, T], H_{x}^{1}\right)}^{\theta}\left\|v_{n}^{j}\right\|_{S\left([0, T], \dot{H}^{\left.\gamma_{c}\right)}\right.}^{\alpha-\theta}\left\|\langle\nabla\rangle v_{n}^{j}\right\|_{S\left([0, T], L^{2}\right)}
\end{aligned}
$$

which, by (2.32), (2.42), and (2.44), implies

$$
\left\|v_{n}^{j}-e^{i\left(t-t_{n}^{j}\right) \Delta} \psi^{j}\left(\cdot-x_{n}^{j}\right)\right\|_{L_{t}^{\infty}\left([0, T], H_{x}^{1}\right)} \rightarrow 0 \text { as } n \rightarrow \infty .
$$

We thus prove (2.43).

Denote

$$
B:=\max \left\{1, \lim _{n \rightarrow \infty} \sup _{t \in[0, T]}\left\|\nabla u_{n}(t)\right\|_{L^{2}}\right\}<\infty .
$$

and let $T^{1}$ the maximal forward time such that

$$
\sup _{t \in\left[0, T^{1}\right]}\left\|\nabla v^{1}(t)\right\|_{L^{2}} \leq 2 B
$$

In what follows, we will show that for all $t \in\left[0, T^{1}\right]$,

$$
\left\|\nabla u_{n}(t)\right\|_{L^{2}}^{2}=\sum_{j=1}^{J}\left\|\nabla v_{n}^{j}(t)\right\|_{L^{2}}^{2}+\left\|\nabla \tilde{W}_{n}^{J}(t)\right\|_{L^{2}}^{2}+o_{J, n}(1),
$$

where $o_{J, n}(1) \rightarrow 0$ as $J, n \rightarrow \infty$ uniformly on $0 \leq t \leq T^{1}$. We see that (2.45) implies $(2.39)$ as $T^{1} \geq T$. In fact, if $T^{1}<T$, then by $(2.45)$,

$$
\sup _{t \in\left[0, T^{1}\right]}\left\|\nabla v^{1}(t)\right\|_{L^{2}}=\sup _{t \in\left[0, T^{1}\right]}\left\|\nabla v_{n}^{1}(t)\right\|_{L^{2}} \leq \sup _{t \in\left[0, T^{1}\right]}\left\|\nabla u_{n}(t)\right\|_{L^{2}} \leq \sup _{t \in[0, T]}\left\|\nabla u_{n}(t)\right\|_{L^{2}} \leq B .
$$

Note that $t_{n}^{1} \equiv 0$. By the continuity, it contradicts the maximality of $T^{1}$. 
We estimate $\left\|v_{n}^{1}\right\|_{S\left(\left[0, T^{1}\right], \dot{H}^{\gamma_{\mathrm{c}}}\right)}$ as follows. For $N \geq 3$, by interpolation between endpoints and Sobolev embedding, we have

$$
\begin{aligned}
\left\|v_{n}^{1}\right\|_{S\left(\left[0, T^{1}\right], \dot{H}^{\gamma_{c}}\right)} & =\left\|v^{1}\right\|_{S\left(\left[0, T^{1}\right], \dot{H}^{\gamma_{c}}\right)} \\
& \lesssim\left\|v^{1}\right\|_{L_{t}^{\frac{2}{1-\gamma_{c}}}\left(\left[0, T^{1}\right], L_{x}^{\frac{2 N}{N-2}}\right)}+\left\|v^{1}\right\|_{L_{t}^{\infty}\left(\left[0, T^{1}\right], L_{x}^{\frac{2 N}{N-2 \gamma_{c}}}\right)} \\
& \left.\lesssim\left\|v^{1}\right\|_{L_{t}^{\frac{2}{1-\gamma_{c}}}\left(\left[0, T^{1}\right], L_{x}^{\frac{2 N}{N-2}}\right)}+\left\|v^{1}\right\|_{L_{t}^{\infty}\left(\left[0, T^{1}\right], L_{x}^{2}\right)}^{1-v^{1} \|^{\gamma_{c}}}{ }_{L_{t}^{\infty}\left(\left[0, T^{1}\right], L_{x}^{N-2}\right.}^{\frac{2 N}{N-2}}\right) \\
& \lesssim\left(T^{1}\right)^{\frac{1-\gamma_{c}}{2}}\left\|\nabla v^{1}\right\|_{L_{t}^{\infty}\left(\left[0, T^{1}\right], L_{x}^{2}\right)}+C\left\|\nabla v^{1}\right\|_{L_{c}^{\infty}\left(\left[0, T^{1}\right], L_{x}^{\frac{2 N}{N-2}}\right)} \\
& \lesssim\left(T^{1}\right)^{\frac{1-\gamma_{c}}{2}} B+C B^{\gamma_{c}} .
\end{aligned}
$$

Here we have use the conservation of mass and the choice of $v^{1}$ to have that for all $t \in\left[0, T^{1}\right]$,

$$
\left\|v^{1}(t)\right\|_{L^{2}}=\lim _{n \rightarrow \infty}\left\|v^{1}\left(-t_{n}^{1}\right)\right\|_{L^{2}}=\lim _{n \rightarrow \infty}\left\|e^{-i t_{n}^{1} \Delta} \psi^{1}\right\|_{L^{2}}=\left\|\psi^{1}\right\|_{L^{2}} \leq\left\|u_{n, 0}\right\|_{L^{2}} \leq 1
$$

When $N=2$, a similar estimate holds by interpolating between $\left(\infty, \frac{2}{1-\gamma_{\mathrm{c}}}\right)$ and $\left(\frac{2}{1-\gamma_{\mathrm{c}}}, r\right)$ with $r$ sufficiently large and using Sobolev embedding. This shows that

$$
\left\|v_{n}^{1}\right\|_{S\left(\left[0, T^{1}\right], \dot{H}^{\gamma_{c}}\right)} \leq C\left(T^{1}, B\right) .
$$

Now we define the approximation

$$
\tilde{u}_{n}^{J}(t, x):=\sum_{j=1}^{J} v_{n}^{j}(t, x)
$$

We have

By (2.34), we have

$$
u_{n, 0}(x)-\tilde{u}_{n}^{J}(0, x)=\tilde{W}_{n}^{J}(x) .
$$

$$
\lim _{J \rightarrow \infty}\left[\lim _{n \rightarrow \infty}\left\|e^{i t \Delta}\left(u_{n, 0}-\tilde{u}_{n}^{J}(0)\right)\right\|_{S\left(\dot{H}^{\gamma_{c}}\right) \cap L_{t}^{\infty}\left(\mathbb{R}, L_{x}^{\frac{2 N}{N-2 \gamma_{c}}}\right)}\right]=0 .
$$

We also have

where

$$
i \partial_{t} \tilde{u}_{n}^{J}+\Delta \tilde{u}_{n}^{J}+|x|^{-b}\left|\tilde{u}_{n}^{J}\right|^{\alpha} \tilde{u}_{n}^{J}=\tilde{e}_{n}^{J},
$$

$$
\tilde{e}_{n}^{J}=\sum_{j=1}^{J} F\left(v_{n}^{j}\right)-F\left(\sum_{j=1}^{J} v_{n}^{j}\right)
$$

with $F(u):=|x|^{-b}|u|^{\alpha} u$. We also have the following properties of the approximate solutions.

Lemma 2.11. The functions $\tilde{u}_{n}^{J}$ and $\tilde{e}_{n}^{J}$ satisfy

$$
\limsup _{n \rightarrow \infty}\left(\left\|\tilde{u}_{n}^{J}\right\|_{L_{t}^{\infty}\left(\left[0, T^{1}\right], H_{x}^{1}\right)}+\left\|\tilde{u}_{n}^{J}\right\|_{S\left(\left[0, T^{1}\right], \dot{H} \gamma_{c}\right)}\right) \lesssim 1
$$

uniformly in $J$ and

$$
\lim _{J \rightarrow \infty} \lim _{n \rightarrow \infty}\left\|\langle\nabla\rangle \tilde{e}_{n}^{J}\right\|_{S^{\prime}\left(\left[0, T^{1}\right], L^{2}\right)}+\left\|\tilde{e}_{n}^{J}\right\|_{S^{\prime}\left(\left[0, T^{1}\right], \dot{H}^{-} \gamma_{c}\right)}=0 .
$$

Proof. The boundedness of $\left\|\tilde{u}_{n}^{J}\right\|_{S\left(\left[0, T^{1}\right], \dot{H}^{\gamma_{c}}\right)}$ follows from (2.41), (2.42), and (2.46). The boundedness of $\left\|\tilde{u}_{n}^{J}\right\|_{L_{t}^{\infty}\left(\left[0, T^{1}\right], L_{x}^{2}\right)}$ follows from $(2.29)$ and the fact that

$$
\left\|v_{n}^{j}(t)\right\|_{L^{2}}=\left\|v^{j}\left(t-t_{n}^{j}\right)\right\|_{L^{2}}=\lim _{n \rightarrow \infty}\left\|v\left(-t_{n}^{j}\right)\right\|_{L^{2}}=\lim _{n \rightarrow \infty}\left\|e^{-i t_{n}^{j} \Delta} \psi^{j}\right\|_{L^{2}}=\left\|\psi^{j}\right\|_{L^{2}}
$$

To see the boundedness of $\left\|\nabla \tilde{u}_{n}^{J}\right\|_{L_{t}^{\infty}\left(\left[0, T^{1}\right], L_{x}^{2}\right)}$, we proceed as follows. For $j \geq J_{0}$, by (2.41), we split $\left[0, T^{1}\right]$ into finite subintervals $I_{k}, k=1, \cdots, M$ such that $\left\|v_{n}^{j}\right\|_{S\left(I_{k}, \dot{H}^{\left.\gamma_{c}\right)}\right.}$ is small. By Duhamel's formula, Strichartz estimates, and Lemma 2.2, we have

$$
\left\|\nabla v_{n}^{j}\right\|_{L_{t}^{\infty}\left(I_{k}, L_{x}^{2}\right)} \lesssim\left\|\nabla v_{n}^{j}\left(t_{k}\right)\right\|_{L^{2}}, \quad I_{k}=\left[t_{k}, t_{k+1}\right], \quad k=1, \cdots, M
$$

Summing over these finite intervals, we get

$$
\left\|\nabla v_{n}^{j}\right\|_{L_{t}^{\infty}\left(\left[0, T^{1}\right], L_{x}^{2}\right)} \lesssim\left\|\nabla v_{n}^{j}(0)\right\|_{L^{2}}
$$

For $2 \leq j \leq J_{0}$, we have from the Duhamel formula, Strichartz estimates, Lemma 2.2, and (2.42), we have

$$
\left\|\nabla v_{n}^{j}\right\|_{L_{t}^{\infty}\left(\left[0, T^{1}\right], L_{x}^{2}\right)} \lesssim\left\|\nabla v_{n}^{j}(0)\right\|_{L^{2}}
$$


for $n$ sufficiently large. Thus we have

$$
\begin{aligned}
\left\|\nabla \tilde{u}_{n}^{J}\right\|_{L_{t}^{\infty}\left(\left[0, T^{1}\right], L_{x}^{2}\right)}^{2} & \leq\left\|\nabla v^{1}\right\|_{L_{t}^{\infty}\left(\left[0, T^{1}\right], L_{x}^{2}\right)}^{2}+\sum_{j=2}^{J}\left\|\nabla v_{n}^{j}\right\|_{L_{t}^{\infty}\left(\left[0, T^{1}\right], L_{x}^{2}\right)}^{2} \\
& \lesssim B^{2}+\sum_{j=2}^{J}\left\|\nabla v_{n}^{j}(0)\right\|_{L^{2}}^{2} \\
& \lesssim B^{2}+\sum_{j=2}^{J}\left\|\nabla \psi^{j}\right\|_{L^{2}}^{2}+o_{n}(1) \\
& \lesssim B^{2}+\left\|\nabla u_{n, 0}\right\|_{L^{2}}^{2}+o_{n}(1) \\
& \lesssim B^{2}+o_{n}(1) .
\end{aligned}
$$

This shows the boundedness of $\left\|\nabla \tilde{u}_{n}^{J}\right\|_{L_{t}^{\infty}\left(\left[0, T^{1}\right], L_{x}^{2}\right)}$ and we prove (2.48). To see (2.49), we follow from the same argument as in [22, Claim 1 (6.23)]. We thus omit the details.

Thanks to (2.47) and Lemma 2.11, the stability given in Lemma 2.4 (see also Remark 2.1) implies

$$
\lim _{J \rightarrow \infty}\left[\lim _{n \rightarrow \infty}\left\|u_{n}-\tilde{u}_{n}^{J}\right\|_{S\left(\left[0, T^{1}\right], \dot{H}^{\gamma_{c}}\right) \cap L_{t}^{\infty}\left(\left[0, T^{1}\right], L_{x}^{\frac{2 N}{N-2 \gamma_{c}}}\right)}\right]=0 .
$$

By interpolating between endpoints and using Sobolev embedding, we infer that

$$
\left\|u_{n}-\tilde{u}_{n}^{J}\right\|_{L_{t}^{\infty}\left(\left[0, T^{1}\right], L_{x}^{\alpha+2}\right) \cap L_{t}^{\infty}\left(\left[0, T^{1}\right], L_{x}^{r}\right)} \lesssim\left\|u_{n}-\tilde{u}_{n}^{J}\right\|_{L_{t}^{\infty}\left(\left[0, T^{1}\right], L_{x}^{\frac{2 N}{N-2 \gamma_{c}}}\right)}\left\|\langle\nabla\rangle\left(u_{n}-\tilde{u}_{n}^{J}\right)\right\|_{L_{t}^{\infty}\left(\left[0, T^{1}\right], L_{x}^{2}\right)} \rightarrow 0
$$

as $J, n \rightarrow \infty$, where $r$ is an exponent satisfying $\frac{2 N}{N-2 \gamma_{c}}<\frac{N(\alpha+2)}{N-b}<r<2^{*}$. This estimate together with (2.35) yield

$$
\left|P\left(u_{n}(t)\right)-P\left(\tilde{u}_{n}^{J}(t)\right)\right| \rightarrow 0
$$

as $J, n \rightarrow \infty$ uniformly on $0 \leq t \leq T^{1}$. On the other hand, we have from the same argument as in [22, Proposition 5.3] using (2.43) that for all $t \in\left[0, T^{1}\right]$,

$$
P\left(\tilde{u}_{n}^{J}(t)\right)=\sum_{j=1}^{J} P\left(v_{n}^{j}(t)\right)+o_{J, n}(1)=\sum_{j=1}^{J} P\left(v_{n}^{j}(t)\right)+P\left(\tilde{W}_{n}^{J}(t)\right)+o_{J, n}(1) .
$$

Here we have used the fact that $P\left(\tilde{W}_{n}^{J}(t)\right)=o_{J, n}(1)$ uniformly on $0 \leq t \leq T^{1}$. In fact, by the Duhamel formula and Lemma 2.2, we have

$$
\left\|\tilde{W}_{n}^{J}(t)\right\|_{S\left(\dot{H}^{\gamma_{c}}\right)} \leq\left\|e^{i t \Delta} \tilde{W}_{n}^{J}\right\|_{S\left(\dot{H}^{\left.\gamma_{c}\right)}\right.}+C\left\|\tilde{W}_{n}^{J}(t)\right\|_{L_{t}^{\infty}\left(\mathbb{R}, H_{x}^{1}\right)}^{\theta}\left\|\tilde{W}_{n}^{J}(t)\right\|_{S\left(\dot{H}^{\gamma_{c}}\right)}^{\alpha+1-\theta}
$$

for some $\theta>0$ sufficiently small. Since $\left\|\tilde{W}_{n}^{J}(t)\right\|_{L_{t}^{\infty}\left(\mathbb{R}, H_{x}^{1}\right)} \lesssim 1$ (by the small data theory), the continuity argument together with (2.28) imply

$$
\lim _{J \rightarrow \infty}\left[\lim _{n \rightarrow \infty}\left\|\tilde{W}_{n}^{J}(t)\right\|_{S\left(\dot{H}^{\gamma_{c}}\right)}\right]=0 .
$$

Thanks to (2.52), Strichartz estimates, and (2.34), we have

$$
\lim _{J \rightarrow \infty}\left[\lim _{n \rightarrow \infty}\left\|\tilde{W}_{n}^{J}(t)\right\|_{L_{t}^{\infty}\left(\mathbb{R}, L_{x}^{\frac{2 N}{N-2 \gamma_{\mathrm{c}}}}\right)}\right]=0
$$

which together with (2.35) yield

$$
\lim _{J \rightarrow \infty}\left[\lim _{n \rightarrow \infty} \sup _{t \in \mathbb{R}} P\left(\tilde{W}_{n}^{J}(t)\right)\right]=0 .
$$

Moreover, by the conservation of energy, we have

$$
\begin{aligned}
E\left(u_{n}(t)\right)=E\left(u_{n, 0}\right) & =\sum_{j=1}^{J} E\left(v_{n}^{j}(0)\right)+E\left(\tilde{W}_{n}^{J}\right)+o_{n}(1) \\
& =\sum_{j=1}^{J} E\left(v_{n}^{j}(t)\right)+E\left(\tilde{W}_{n}^{J}(t)\right)+o_{J, n}(1) .
\end{aligned}
$$

Collecting (2.50), (2.51), and (2.53), we prove (2.45). The proof is complete. 
We come back to the proof of Proposition 2.9. We will consider two cases.

Case 1. More than one non-zero profiles. We have

$$
M\left(v_{n}^{j}(t)\right)=M\left(v_{n}^{j}(0)\right)=M\left(e^{-i t_{n}^{j} \Delta} \psi^{j}\right)=M\left(\psi^{j}\right)<1, \quad \forall j \geq 1 .
$$

By (2.23) and (2.40), we have

$$
\sup _{t \in[0, \infty)} P\left(v_{n}^{j}(t)\right)\left[M\left(v_{n}^{j}(t)\right)\right]^{\sigma_{\mathrm{c}}}<A, \quad \forall j \geq 1 .
$$

Here we note that by $(2.39),\left\|\nabla v_{n}^{j}(t)\right\|_{L^{2}}$ is bounded uniformly which implies $v_{n}^{j}$ exists globally in time. By Lemma 2.8, we have $E\left(v_{n}^{j}(t)\right) \geq 0$, hence

$$
E\left(v_{n}^{j}(t)\right)\left[M\left(v_{n}^{j}(t)\right)\right]^{\sigma_{\mathrm{c}}}<\delta_{\mathrm{c}}, \quad \forall j \geq 1 .
$$

By Item (1) (see after (2.22)), we have

We can approximate $u_{n}$ by

$$
\left\|v_{n}^{j}\right\|_{S\left([0, \infty), \dot{H}^{\left.\gamma_{c}\right)}\right.}<\infty, \quad \forall j \geq 1
$$

$$
u_{n}^{J}(t, x):=\sum_{j=1}^{J} v_{n}^{j}(t)
$$

and get for $J$ sufficiently large that

which is a contradiction.

$$
\left\|u_{n}\right\|_{S\left([0, \infty), \dot{H}^{\gamma_{c}}\right)}<\infty
$$

Case 2. Only one non-zero profile. We must have only one non-zero profile, i.e.,

$$
u_{n, 0}(x)=e^{-i t_{n}^{1} \Delta} \psi^{1}\left(x-x_{n}^{1}\right)+W_{n}(x), \quad \lim _{n \rightarrow \infty}\left\|e^{i t \Delta} W_{n}\right\|_{S\left([0, \infty), \dot{H} \gamma_{c}\right)}=0 .
$$

We note that $t_{n}^{1}$ cannot tend to $-\infty$. Indeed, if $t_{n}^{1} \rightarrow-\infty$, then we have

$$
\left\|e^{i t \Delta} u_{n, 0}\right\|_{S\left([0, \infty), \dot{H}^{\gamma_{c}}\right)} \leq\left\|e^{i t \Delta} \psi^{1}\right\|_{S\left(\left[-t_{n}^{1}, \infty\right), \dot{H}^{\gamma_{c}}\right)}+\left\|e^{i t \Delta} W_{n}\right\|_{S\left([0, \infty), \dot{H}^{\gamma_{c}}\right)} \rightarrow 0
$$

as $n \rightarrow \infty$. By the Duhamel formula, Lemma 2.2, and the continuity argument, $\left\|u_{n}\right\|_{S\left([0, \infty), \dot{H}^{\gamma_{\mathrm{c}}}\right)}<\infty$ for $n$ sufficiently large which is a contradiction.

We claim that $x_{n}^{1} \equiv 0$. Otherwise, if $\left|x_{n}^{1}\right| \rightarrow \infty$, then, by Lemma 2.7, for $n$ large, there exist global solutions $v_{n}$ to (1.2) satisfying $v_{n}(0, x)=e^{-i t_{n}^{1} \Delta} \psi^{1}\left(x-x_{n}^{1}\right)$. Moreover, $v_{n}$ scatters in $H^{1}$ in both directions. In particular, $\left\|v_{n}\right\|_{S\left(\dot{H}^{\gamma_{c}}\right)}<\infty$. Again, by the long time perturbation, we show that $\left\|u_{n}\right\|_{S\left([0, \infty), \dot{H}^{\left.\gamma_{c}\right)}\right.}<\infty$ for $n$ sufficiently large which is a contradiction.

Let $v^{1}$ be the nonlinear profile associated to $\psi^{1}$ and $t_{n}^{1}$, we have

$$
u_{n, 0}(x)=v^{1}\left(-t_{n}^{1}, x\right)+\tilde{W}_{n}(x) .
$$

Set $v_{n}^{1}(t)=v^{1}\left(t-t_{n}^{1}\right)$. Arguing as above, we have

$$
M\left(v_{n}^{1}(t)\right) \leq 1, \quad \sup _{t \in[0, \infty)} P\left(v_{n}^{1}(t)\right) \leq A, \quad E\left(v_{n}^{1}(t)\right) \leq \delta_{\mathrm{c}}, \quad \lim _{n \rightarrow \infty}\left\|\tilde{W}_{n}(t)\right\|_{S\left(\dot{H}^{\gamma_{c}}\right)}=0 .
$$

We infer that $M\left(v_{n}^{1}(t)\right)=1$ and $E\left(v_{n}^{1}(t)\right)=\delta_{\mathrm{c}}$. Otherwise, if $M\left(v_{n}^{1}(t)\right)<1$, then

$$
\sup _{t \in[0, \infty)} P\left(v_{n}^{1}(t)\right)\left[M\left(v_{n}^{1}(t)\right)\right]^{\sigma_{\mathrm{c}}}<A, \quad E\left(v_{n}^{1}\right)\left[M\left(v_{n}^{1}\right)\right]^{\sigma_{\mathrm{c}}}<\delta_{\mathrm{c}}
$$

By Item (1) (see again after (2.22)), we have $\left\|v_{n}^{1}\right\|_{S\left([0, \infty), \dot{H}^{\left.\gamma_{c}\right)}\right.}<\infty$. Thus we get a contradiction by the long time perturbation argument.

Now we define $u_{\mathrm{c}}$ the solution to (1.2) with initial data $\left.u_{\mathrm{c}}\right|_{t=0}=v^{1}(0)$. We have

$$
\begin{gathered}
M\left(u_{\mathrm{c}}\right)=M\left(v^{1}(0)\right)=M\left(v^{1}\left(t-t_{n}^{1}\right)\right)=M\left(v_{n}^{1}(t)\right)=1, \\
E\left(u_{\mathrm{c}}\right)=E\left(v^{1}(0)\right)=E\left(v^{1}\left(t-t_{n}^{1}\right)\right)=E\left(v_{n}^{1}(t)\right)=\delta_{\mathrm{c}} .
\end{gathered}
$$

Moreover,

$$
\sup _{t \in[0, \infty)} P\left(u_{\mathrm{c}}(t)\right)=\sup _{t \in[0, \infty)} P\left(v^{1}(t)\right)=\sup _{t \in\left[t_{n}^{1}, \infty\right)} P\left(v^{1}\left(t-t_{n}^{1}\right)\right)=\sup _{t \in\left[t_{n}^{1}, \infty\right)} P\left(v_{n}^{1}(t)\right) \leq A .
$$

By the definition of $\delta_{\mathrm{c}}$, we must have $\left\|u_{\mathrm{c}}\right\|_{S\left([0, \infty), \dot{H}^{\left.\gamma_{\mathrm{c}}\right)}\right.}=\infty$. This shows (2.24).

By the same argument as in the proof of [22, Proposition 6.3], we show that the set

$$
\mathcal{K}:=\left\{u_{\mathrm{c}}(t): t \in[0, \infty)\right\}
$$

is precompact in $H^{1}$. 
Step 3. Exclusion of the critical solution. Thanks to the above compactness result, the standard rigidity argument using localized virial estimates and Lemma 2.8 shows that $u_{\mathrm{c}} \equiv 0$ which contradicts (2.24). We refer the reader to [22, Section 7] for more details. The proof of Proposition 2.9 is now complete. This also ends the proof of Theorem 1.1.

\section{BLOW-UP CRITERION}

In this section, we give the proof of the blow-up criterion given in Theorem 1.2. Let us recall the following virial identity (see e.g., [11]).

Lemma 3.1. Let $\varphi: \mathbb{R}^{N} \rightarrow \mathbb{R}$ be a sufficiently smooth and decaying function. Let $u$ be a solution to (1.2) defined on the maximal forward time interval of existence $\left[0, T^{*}\right)$. Define

$$
V_{\varphi}(t):=\int \varphi(x)|u(t, x)|^{2} d x
$$

Then we have for all $t \in\left[0, T^{*}\right)$,

$$
V_{\varphi}^{\prime}(t)=2 \operatorname{Im} \int \nabla \varphi(x) \cdot \nabla u(t, x) \bar{u}(t, x) d x
$$

and

$$
\begin{aligned}
V_{\varphi}^{\prime \prime}(t)= & -\int \Delta^{2} \varphi(x)|u(t, x)|^{2} d x+4 \sum_{j, k=1}^{N} \operatorname{Re} \int \partial_{j k}^{2} \varphi(x) \partial_{j} \bar{u}(t, x) \partial_{k} u(t, x) d x \\
& -\frac{2 \alpha}{\alpha+2} \int|x|^{-b} \Delta \varphi(x)|u(t, x)|^{\alpha+2} d x+\frac{4}{\alpha+2} \int \nabla \varphi(x) \cdot \nabla\left(|x|^{-b}\right)|u(t, x)|^{\alpha+2} d x .
\end{aligned}
$$

Remark 3.1. (1) In the case $\varphi(x)=|x|^{2}$, we have

where $G(f)$ is as in (1.15).

$$
\frac{d^{2}}{d t^{2}}\|x u(t)\|_{L^{2}}^{2}=8 G(u(t))
$$

(2) In the case $\varphi$ is radially symmetric, it follows from

$$
\partial_{j}=\frac{x_{j}}{r} \partial_{r}, \quad \partial_{j k}^{2}=\left(\frac{\delta_{j k}}{r}-\frac{x_{j} x_{k}}{r^{3}}\right) \partial_{r}+\frac{x_{j} x_{k}}{r^{2}} \partial_{r}^{2}
$$

that

$$
\begin{aligned}
& \sum_{j, k=1}^{N} \operatorname{Re} \int \partial_{j k}^{2} \varphi(x) \partial_{j} \bar{u}(t, x) \partial_{k} u(t, x) d x \\
& \quad=\int \frac{\varphi^{\prime}(r)}{r}|\nabla u(t, x)|^{2} d x+\int\left(\frac{\varphi^{\prime \prime}(r)}{r^{2}}-\frac{\varphi^{\prime}(r)}{r^{3}}\right)|x \cdot \nabla u(t, x)|^{2} d x .
\end{aligned}
$$

In particular, we have

$$
\begin{aligned}
& V_{\varphi}^{\prime \prime}(t) \\
& =-\int \Delta^{2} \varphi(x)|u(t, x)|^{2} d x+4 \int \frac{\varphi^{\prime}(r)}{r}|\nabla u(t, x)|^{2} d x+4 \int\left(\frac{\varphi^{\prime \prime}(r)}{r^{2}}-\frac{\varphi^{\prime}(r)}{r^{3}}\right)|x \cdot \nabla u(t, x)|^{2} d x \\
& \quad-\frac{2 \alpha}{\alpha+2} \int|x|^{-b} \Delta \varphi(x)|u(t, x)|^{\alpha+2}-\frac{4 b}{\alpha+2} \int|x|^{-b} \frac{\varphi^{\prime}(r)}{r}|u(t, x)|^{\alpha+2} d x .
\end{aligned}
$$

(3) Denote $x=\left(y, x_{N}\right)$ with $y=\left(x_{1}, \cdots, x_{N-1}\right) \in \mathbb{R}^{N-1}$ and $x_{N} \in \mathbb{R}$. Let $\psi: \mathbb{R}^{N-1} \rightarrow \mathbb{R}$ be a sufficiently smooth decaying function. Set $\varphi(x)=\varphi\left(y, x_{N}\right)=\psi(y)+x_{N}^{2}$. We have

$$
V_{\varphi}^{\prime}(t)=2 \operatorname{Im} \int\left(\nabla_{y} \psi(y) \cdot \nabla_{y} u(t, x)+2 x_{N} \partial_{N} u(t, x)\right) \bar{u}(t, x) d x
$$

and

$$
\begin{aligned}
V_{\varphi}^{\prime \prime}(t)= & -\int \Delta_{y}^{2} \psi(y)|u(t, x)|^{2} d x+4 \sum_{j, k=1}^{N-1} \operatorname{Re} \int \partial_{j k}^{2} \psi(y) \partial_{j} \bar{u}(t, x) \partial_{k} u(t, x) d x \\
& -\frac{2 \alpha}{\alpha+2} \int|x|^{-b} \Delta_{y} \psi(y)|u(t, x)|^{\alpha+2} d x-\frac{4 b}{\alpha+2} \int \nabla_{y} \psi(y) \cdot y|x|^{-b-2}|u(t, x)|^{\alpha+2} d x \\
& +8\left\|\partial_{N} u(t)\right\|_{L^{2}}^{2}-\frac{4 \alpha}{\alpha+2} \int|x|^{-b}|u(t, x)|^{\alpha+2} d x-\frac{8 b}{\alpha+2} \int x_{N}^{2}|x|^{-b-2}|u(t, x)|^{\alpha+2} d x
\end{aligned}
$$


Let $\chi$ be a smooth radial function satisfying

Given $R>1$, we define the radial function

$$
\chi(x)=\chi(r)=\left\{\begin{array}{cl}
r^{2} & \text { if } \quad r \leq 1, \\
0 & \text { if } \quad r \geq 2,
\end{array} \quad \chi^{\prime \prime}(r) \leq 2 \quad \forall r=|x| \geq 0 .\right.
$$

$$
\varphi_{R}(x):=R^{2} \chi(x / R) .
$$

We have the following localized virial estimate.

Proposition 3.2. Let $N \geq 1,0<b<\min \{2, N\}$, and $\frac{4-2 b}{N}<\alpha<\alpha(N)$. Let $u$ be a solution to (1.2) defined on the maximal forward time interval of existence $\left[0, T^{*}\right)$. Let $\varphi_{R}$ be as in $(3.3)$ and define $V_{\varphi_{R}}(t)$ as in (3.1). Then we have for all $t \in\left[0, T^{*}\right)$,

$$
V_{\varphi_{R}}^{\prime}(t)=2 \operatorname{Im} \int \nabla \varphi_{R}(x) \cdot \nabla u(t, x) \bar{u}(t, x) d x
$$

and

$$
V_{\varphi_{R}}^{\prime \prime}(t) \leq 8 G(u(t))+C R^{-2}+C R^{-b}\|u(t)\|_{H^{1}}^{\alpha+2}
$$

where $G$ is as in (1.15) and some constant $C>0$ independent of $R$.

Proof. It follows from (3.2) that

$$
\begin{aligned}
V_{\varphi_{R}}^{\prime \prime}(t)= & 8 G(u(t))-8\|\nabla u(t)\|_{L^{2}}^{2}+\frac{4(N \alpha+2 b)}{\alpha+2} \int|x|^{-b}|u(t, x)|^{\alpha+2} d x \\
& -\int \Delta^{2} \varphi_{R}(x)|u(t, x)|^{2} d x+4 \int \frac{\varphi_{R}^{\prime}(r)}{r}|\nabla u(t, x)|^{2} d x \\
& +4 \int\left(\frac{\varphi_{R}^{\prime \prime}(r)}{r^{2}}-\frac{\varphi_{R}^{\prime}(r)}{r^{3}}\right)|x \cdot \nabla u(t, x)|^{2} d x \\
& -\frac{2 \alpha}{\alpha+2} \int|x|^{-b} \Delta \varphi_{R}(x)|u(t, x)|^{\alpha+2} d x-\frac{4 b}{\alpha+2} \int|x|^{-b} \frac{\varphi_{R}^{\prime}(r)}{r}|u(t, x)|^{\alpha+2} d x .
\end{aligned}
$$

As $\left\|\Delta^{2} \varphi_{R}\right\|_{L^{\infty}} \lesssim R^{-2}$, the conservation of mass implies that

$$
\left.\left|\int \Delta^{2} \varphi_{R}(x)\right| u(t, x)\right|^{2} d x \mid \lesssim R^{-2}\|u(t)\|_{L^{2}}^{2} \lesssim R^{-2}
$$

By the Cauchy-Schwarz inequality $|x \cdot \nabla u| \leq|x||\nabla u|=r|\nabla u|$ and the fact $\varphi_{R}^{\prime \prime}(r) \leq 2$, we see that

$$
\begin{gathered}
4 \int \frac{\varphi_{R}^{\prime}(r)}{r}|\nabla u(t, x)|^{2} d x+4 \int\left(\frac{\varphi_{R}^{\prime \prime}(r)}{r^{2}}-\frac{\varphi_{R}^{\prime}(r)}{r^{3}}\right)|x \cdot \nabla u(t, x)|^{2} d x-8\|\nabla u(t)\|_{L^{2}}^{2} \\
\leq 4 \int\left(\frac{\varphi_{R}^{\prime}(r)}{r}-2\right)|\nabla u(t, x)|^{2} d x+4 \int \frac{1}{r^{2}}\left(2-\frac{\varphi_{R}^{\prime}(r)}{r}\right)|x \cdot \nabla u(t, x)|^{2} d x \leq 0 .
\end{gathered}
$$

Moreover,

$$
\begin{aligned}
& \frac{4(N \alpha+2 b)}{\alpha+2} \int|x|^{-b}|u(t, x)|^{\alpha+2} d x-\frac{2 \alpha}{\alpha+2} \int|x|^{-b} \Delta \varphi_{R}(x)|u(t, x)|^{\alpha+2} d x \\
&-\frac{4 b}{\alpha+2} \int|x|^{-b} \frac{\varphi_{R}^{\prime}(r)}{r}|u(t, x)|^{\alpha+2} d x \\
&=\frac{2 \alpha}{\alpha+2} \int|x|^{-b}\left(2 N-\Delta \varphi_{R}(x)\right)|u(t, x)|^{\alpha+2} d x+\frac{4 b}{\alpha+2} \int|x|^{-b}\left(2-\frac{\varphi_{R}^{\prime}(r)}{r}\right)|u(t, x)|^{\alpha+2} d x
\end{aligned}
$$

Since $\Delta \varphi_{R} \leq 2 N, \frac{\varphi_{R}^{\prime}(r)}{r} \leq 2, \Delta \varphi_{R}(x)=2 N$, and $\frac{\varphi_{R}^{\prime}(r)}{r}=2$ for $r=|x| \leq R$, the above quantity is bounded by

$$
C \int_{|x| \geq R}|x|^{-b}|u(t)|^{\alpha+2} d x \leq C R^{-b}\|u(t)\|_{L^{\alpha+2}}^{\alpha+2} \leq C R^{-b}\|u(t)\|_{H^{1}}^{\alpha+2}
$$

where the last inequality follows from the Sobolev embedding as $\alpha<\alpha(N)$. Collecting the above estimates, we end the proof.

Proof of Theorem 1.2. Let $u:\left[0, T^{*}\right) \times \mathbb{R}^{N} \rightarrow \mathbb{C}$ be a solution to (1.2) satisfying (1.14). If $T^{*}<\infty$, then we are done. If $T^{*}=\infty$, then we show that there exists $t_{n} \rightarrow \infty$ such that $\left\|\nabla u\left(t_{n}\right)\right\|_{L^{2}} \rightarrow \infty$ as $n \rightarrow \infty$. Assume by contradiction that it does not hold, i.e., $\sup _{t \in[0, \infty)}\|\nabla u(t)\|_{L^{2}} \leq C_{0}$ for some $C_{0}>0$. By the conservation of mass, we have

$$
\sup _{t \in[0, \infty)}\|u(t)\|_{H^{1}} \leq C_{1}
$$


for some $C_{1}>0$.

By Proposition 3.2, (1.14), and (3.4), we have for all $t \in[0, \infty)$,

$$
V_{\varphi_{R}}^{\prime \prime}(t) \leq 8 G(u(t))+C R^{-2}+C R^{-b}\|u(t)\|_{L^{2}}^{\alpha+2} \leq-8 \delta+C R^{-2}+C R^{-b} C_{1}^{\alpha+2} .
$$

By taking $R>1$ sufficiently large, we have for all $t \in[0, \infty)$,

$$
V_{\varphi_{R}}^{\prime \prime}(t) \leq-4 \delta
$$

Integrating this estimate, there exists $t_{0}>0$ sufficiently large such that $V_{\varphi_{R}}\left(t_{0}\right)<0$ which is impossible. This finishes the first part of Theorem 1.2.

If we assume in addition that $u$ has finite variance, i.e., $u(t) \in L^{2}\left(|x|^{2} d x\right)$ for all $t \in\left[0, T^{*}\right)$, then we have $T^{*}<\infty$. In fact, it follows from Remark 3.1 and (1.14) that

$$
\frac{d^{2}}{d t^{2}}\|x u(t)\|_{L^{2}}^{2}=8 G(u(t)) \leq-8 \delta
$$

for all $t \in\left[0, T^{*}\right)$. The convexity argument of Glassey [30] implies $T^{*}<\infty$.

\section{LONG TIME DYNAMICS}

In this section, we give the proofs of long time dynamics of $H^{1}$-solutions given in Theorems 1.3, 1.4 and 1.5 .

Proof of Theorem 1.3. We will consider separately two cases.

Case 1. Global existence and energy scattering. Let $u_{0} \in H^{1}$ satisfy (1.7) and (1.8). Let us prove (1.16). To see this, we first claim that there exists $\rho=\rho\left(u_{0}, Q\right)>0$ such that

$$
\|\nabla u(t)\|_{L^{2}}\|u(t)\|_{L^{2}}^{\sigma_{c}} \leq(1-\rho)\|\nabla Q\|_{L^{2}}\|Q\|_{L^{2}}^{\sigma_{c}}
$$

for all $t \in\left(-T_{*}, T^{*}\right)$. We assume (4.1) for the moment and prove (1.16). By (2.3) and (4.1), we have

$$
\begin{aligned}
P(u(t))[M(u(t))]^{\sigma_{c}} & \leq C_{\mathrm{opt}}\|\nabla u(t)\|_{L^{2}}^{\frac{N \alpha+2 b}{2}}\|u(t)\|_{L^{2}}^{\frac{4-2 b-(N-2) \alpha}{2}+2 \sigma_{c}} \\
& =C_{\mathrm{opt}}\left(\|\nabla u(t)\|_{L^{2}}\|u(t)\|_{L^{2}}^{\sigma_{c}}\right)^{\frac{N \alpha+2 b}{2}} \\
& \leq C_{\mathrm{opt}}(1-\rho)^{\frac{N \alpha+2 b}{2}}\left(\|\nabla Q\|_{L^{2}}\|Q\|_{L^{2}}^{\sigma_{c}}\right)^{\frac{N \alpha+2 b}{2}}
\end{aligned}
$$

for all $t \in\left(-T_{*}, T^{*}\right)$. By $(2.5)$ and $(2.4)$, we get

$$
P(u(t))[M(u(t))]^{\sigma_{c}} \leq \frac{2(\alpha+2)}{N \alpha+2 b}(1-\rho)^{\frac{N \alpha+2 b}{2}}\left(\|\nabla Q\|_{L^{2}}\|Q\|_{L^{2}}^{\sigma_{c}}\right)^{2}=(1-\rho)^{\frac{N \alpha+2 b}{2}} P(Q)[M(Q)]^{\sigma_{c}}
$$

for all $t \in\left(-T_{*}, T^{*}\right)$ which shows (1.16). By Theorem 1.1, the solution exists globally in time. Moreover, if $N \geq 2$ and $0<b<\min \left\{2, \frac{N}{2}\right\}$, then the solution scatters in $H^{1}$ in both directions.

Let us now prove the claim (4.1). By the definition of energy and (2.3), we have

$$
\begin{aligned}
E(u(t))[M(u(t))]^{\sigma_{c}} & \geq \frac{1}{2}\left(\|\nabla u(t)\|_{L^{2}}\|u(t)\|_{L^{2}}^{\sigma_{c}}\right)^{2}-\frac{C_{\mathrm{opt}}}{\alpha+2}\|\nabla u(t)\|_{L^{2}}^{\frac{N \alpha+2 b}{2^{2}}}\|u(t)\|_{L^{2}}^{\frac{4-2 b-(N-2) \alpha}{2}+2 \sigma_{c}} \\
& =F\left(\|\nabla u(t)\|_{L^{2}}\|u(t)\|_{L^{2}}^{\sigma_{c}}\right),
\end{aligned}
$$

where

$$
F(\lambda):=\frac{1}{2} \lambda^{2}-\frac{C_{\mathrm{opt}}}{\alpha+2} \lambda^{\frac{N \alpha+2 b}{2}} .
$$

Using (2.4), (2.5) and (2.7), we see that

$$
F\left(\|\nabla Q\|_{L^{2}}\|Q\|_{L^{2}}^{\sigma_{c}}\right)=\frac{N \alpha-4+2 b}{2(N \alpha+2 b)}\left(\|\nabla Q\|_{L^{2}}\|Q\|_{L^{2}}^{\sigma_{c}}\right)^{2}=E(Q)[M(Q)]^{\sigma_{c}} .
$$

It follows from (1.7), (4.2) and the conservation of mass and energy that

$$
F\left(\|\nabla u(t)\|_{L^{2}}\|u(t)\|_{L^{2}}^{\sigma_{c}}\right) \leq E\left(u_{0}\right)\left[M\left(u_{0}\right)\right]^{\sigma_{c}}<E(Q)[M(Q)]^{\sigma_{c}}=F\left(\|\nabla Q\|_{L^{2}}\|Q\|_{L^{2}}^{\sigma_{c}}\right)
$$

for all $t \in\left(-T_{*}, T^{*}\right)$. By (1.8), the continuity argument implies

$$
\|\nabla u(t)\|_{L^{2}}\|u(t)\|_{L^{2}}^{\sigma_{c}}<\|\nabla Q\|_{L^{2}}\|Q\|_{L^{2}}^{\sigma_{c}}
$$

for all $t \in\left(-T_{*}, T^{*}\right)$. Next, using (1.7), we take $\vartheta=\vartheta\left(u_{0}, Q\right)>0$ such that

$$
E\left(u_{0}\right)\left[M\left(u_{0}\right)\right]^{\sigma_{c}} \leq(1-\vartheta) E(Q)[M(Q)]^{\sigma_{c}} .
$$

Using

$$
E(Q)[M(Q)]^{\sigma_{\mathrm{c}}}=\frac{N \alpha-4+2 b}{2(N \alpha+2 b)}\left(\|\nabla Q\|_{L^{2}}\|Q\|_{L^{2}}^{\sigma_{\mathrm{c}}}\right)^{2}=\frac{N \alpha-4+2 b}{4(\alpha+2)}\left(\|\nabla Q\|_{L^{2}}\|Q\|_{L^{2}}^{\sigma_{\mathrm{c}}}\right)^{\frac{N \alpha+2 b}{2}},
$$


we we infer from (4.2) and (4.4) that

$$
\frac{N \alpha+2 b}{N \alpha-4+2 b}\left(\frac{\|\nabla u(t)\|_{L^{2}}\|u(t)\|_{L^{2}}^{\sigma_{c}}}{\|\nabla Q\|_{L^{2}}\|Q\|_{L^{2}}^{\sigma_{c}}}\right)^{2}-\frac{4}{N \alpha-4+2 b}\left(\frac{\|\nabla u(t)\|_{L^{2}}\|u(t)\|_{L^{2}}^{\sigma_{c}}}{\|\nabla Q\|_{L^{2}}\|Q\|_{L^{2}}^{\sigma_{c}}}\right)^{\frac{N \alpha+2 b}{2}} \leq 1-\vartheta
$$

for all $t \in\left(-T_{*}, T^{*}\right)$. Let us consider the function

$$
G(\lambda):=\frac{N \alpha+2 b}{N \alpha-4+2 b} \lambda^{2}-\frac{4}{N \alpha-4+2 b} \lambda^{\frac{N \alpha+2 b}{2}}
$$

with $0<\lambda<1$ due to (4.3). We see that $G$ is strictly increasing on $(0,1)$ with $G(0)=0$ and $G(1)=1$. It follows from (4.6) that there exists $\rho>0$ depending on $\vartheta$ such that $\lambda \leq 1-\rho$ which is (4.1). This finishes the first part of Theorem 1.3.

Case 2. Blow-up. Let $u_{0} \in H^{1}$ satisfy (1.7) and (1.10). Let us prove (1.17). By the same argument as above using (1.10) instead of (1.8), we have

$$
\|\nabla u(t)\|_{L^{2}}\|u(t)\|_{L^{2}}^{\sigma_{c}}>\|\nabla Q\|_{L^{2}}\|Q\|_{L^{2}}^{\sigma_{c}}
$$

for all $t \in\left(-T_{*}, T^{*}\right)$. Let $\vartheta$ be as in (4.4). By the conservation laws of mass and energy together with (4.7) and (2.7), we have

$$
\begin{aligned}
G(u(t))[M(u(t))]^{\sigma_{\mathrm{c}}} & =\|\nabla u(t)\|_{L^{2}}^{2}\|u(t)\|^{2 \sigma_{\mathrm{c}}}-\frac{N \alpha+2 b}{2(\alpha+2)} P(u(t))[M(u(t))]^{\sigma_{\mathrm{c}}} \\
& =\frac{N \alpha+2 b}{2} E(u(t))[M(u(t))]^{\sigma_{\mathrm{c}}}-\frac{N \alpha-4+2 b}{4}\left(\|\nabla u(t)\|_{L^{2}}\|u(t)\|_{L^{2}}^{\sigma_{\mathrm{c}}}\right)^{2} \\
& \leq \frac{N \alpha+2 b}{2}(1-\vartheta) E(Q)[M(Q)]^{\sigma_{\mathrm{c}}}-\frac{N \alpha-4+2 b}{4}\left(\|\nabla Q\|_{L^{2}}\|Q\|_{L^{2}}^{\sigma_{\mathrm{c}}}\right)^{2} \\
& =-\frac{N \alpha-4+2 b}{4} \vartheta\left(\|\nabla Q\|_{L^{2}}\|Q\|_{L^{2}}^{\sigma_{\mathrm{c}}}\right)^{2}
\end{aligned}
$$

for all $t \in\left(-T_{*}, T^{*}\right)$. This shows (1.17) with

$$
\delta:=\frac{N \alpha-4+2 b}{4} \vartheta\|\nabla Q\|_{L^{2}}^{2}\left(\frac{M(Q)}{M\left(u_{0}\right)}\right)^{\sigma_{\mathrm{c}}}>0 .
$$

By Theorem 1.2, the corresponding solution either blows up in finite time, or there exists a time sequence $\left(t_{n}\right)_{n \geq 1}$ satisfying $\left|t_{n}\right| \rightarrow \infty$ such that $\left\|\nabla u\left(t_{n}\right)\right\|_{L^{2}} \rightarrow \infty$ as $n \rightarrow \infty$.

- Finite variance data. If we assume in addition that $u_{0} \in \Sigma$, then the corresponding solution blows up in finite time. It directly follows from Theorem 1.2.

- Radially symmetric data. If we assume in addition that $N \geq 2, \alpha \leq 4$, and $u_{0}$ is radially symmetric, then the corresponding solution blows up in finite time. This result was shown in [11]. Note that in [11], $\alpha$ is assumed to be strictly smaller than 4. However, a closer look at the proof of [11], we see that $\alpha=4$ is allowed.

- Cylindrically symmetric data. If we assume in addition that $N \geq 3, \alpha \leq 2$, and $u_{0} \in \Sigma_{N}$ (see (1.18)), then the corresponding solution blows up in finite time. To this end, let $\eta$ be a smooth radial function satisfying

$$
\eta(y)=\eta(\tau)=\left\{\begin{array}{ccc}
\tau^{2} & \text { if } \quad \tau \leq 1, \\
0 & \text { if } \quad \tau \geq 2,
\end{array} \quad \eta^{\prime \prime}(\tau) \leq 2, \quad \forall \tau=|y| \geq 0\right.
$$

Given $R>1$, we define the radial function

$$
\psi_{R}(y):=R^{2} \eta(y / R)
$$

Set

$$
\varphi_{R}(x):=\psi_{R}(y)+x_{N}^{2}
$$

Applying Remark 3.1, we have

$$
V_{\varphi_{R}}^{\prime}(t)=2 \operatorname{Im} \int\left(\nabla_{y} \psi_{R}(y) \cdot \nabla_{y} u(t, x)+2 x_{N} \partial_{N} u(t, x)\right) \bar{u}(t, x) d x
$$


and

$$
\begin{aligned}
V_{\varphi_{R}}^{\prime \prime}(t)= & -\int \Delta_{y}^{2} \psi_{R}(y)|u(t, x)|^{2} d x+4 \sum_{j, k=1}^{N-1} \operatorname{Re} \int \partial_{j k}^{2} \psi_{R}(y) \partial_{j} \bar{u}(t, x) \partial_{k} u(t, x) d x \\
& -\frac{2 \alpha}{\alpha+2} \int|x|^{-b} \Delta_{y} \psi_{R}(y)|u(t, x)|^{\alpha+2} d x-\frac{4 b}{\alpha+2} \int|y|^{2} \frac{\psi_{R}^{\prime}(\tau)}{\tau}|x|^{-b-2}|u(t, x)|^{\alpha+2} d x \\
& +8\left\|\partial_{N} u(t)\right\|_{L^{2}}^{2}-\frac{4 \alpha}{\alpha+2} \int|x|^{-b}|u(t, x)|^{\alpha+2} d x-\frac{8 b}{\alpha+2} \int x_{N}^{2}|x|^{-b-2}|u(t, x)|^{\alpha+2} d x
\end{aligned}
$$

We can rewrite it as

$$
\begin{aligned}
V_{\varphi_{R}}^{\prime \prime}(t)= & 8 G(u(t))-8\left\|\nabla_{y} u(t)\right\|_{L^{2}}^{2}+\frac{4((N-1) \alpha+2 b)}{\alpha+2} P(u(t)) \\
& -\int \Delta_{y}^{2} \psi_{R}(y)|u(t, x)|^{2} d x+4 \sum_{j, k=1}^{N-1} \operatorname{Re} \int \partial_{j k}^{2} \psi_{R}(y) \partial_{j} \bar{u}(t, x) \partial_{k} u(t, x) d x \\
& -\frac{2 \alpha}{\alpha+2} \int \Delta_{y} \psi_{R}(y)|x|^{-b}|u(t, x)|^{\alpha+2} d x-\frac{4 b}{\alpha+2} \int|y|^{2} \frac{\psi_{R}^{\prime}(\tau)}{\tau}|x|^{-b-2}|u(t, x)|^{\alpha+2} d x \\
& -\frac{8 b}{\alpha+2} \int x_{N}^{2}|x|^{-b-2}|u(t, x)|^{\alpha+2} d x
\end{aligned}
$$

Rewriting it further, we get

$$
\begin{aligned}
V_{\varphi_{R}}^{\prime \prime}(t)= & 8 G(u(t))-8\left\|\nabla_{y} u(t)\right\|_{L^{2}}^{2}+4 \sum_{j, k=1}^{N-1} \operatorname{Re} \int \partial_{j k}^{2} \psi_{R}(y) \partial_{j} \bar{u}(t, x) \partial_{k} u(t, x) d x \\
& -\int \Delta_{y}^{2} \psi_{R}(y)|u(t, x)|^{2} d x+\frac{2 \alpha}{\alpha+2} \int\left(2(N-1)-\Delta_{y} \psi_{R}(y)\right)|x|^{-b}|u(t, x)|^{\alpha+2} d x \\
& +\frac{4 b}{\alpha+2} \int\left(2|x|^{2}-\frac{\psi_{R}^{\prime}(\tau)}{\tau}|y|^{2}-2 x_{N}^{2}\right)|x|^{-b-2}|u(t, x)|^{\alpha+2} d x
\end{aligned}
$$

Since $u$ is radially symmetric with respect to the first $N-1$ variables, we use the fact that

$$
\partial_{j}=\frac{y_{j}}{\tau} \partial_{\tau}, \quad \partial_{j k}^{2}=\left(\frac{\delta_{j k}}{\tau}-\frac{y_{j} y_{k}}{\tau^{3}}\right) \partial_{\tau}+\frac{y_{j} y_{k}}{\tau^{2}} \partial_{\tau}^{2}, \quad \tau=|y|, \quad j, k=1, \cdots, N-1
$$

to have

$$
\sum_{j, k=1}^{N-1} \partial_{j k}^{2} \psi_{R}(y) \partial_{j} \bar{u}(t, x) \partial_{k} u(t, x)=\psi_{R}^{\prime \prime}(\tau)\left|\partial_{\tau} u(t, x)\right|^{2} \leq 2\left|\partial_{\tau} u(t, x)\right|^{2}=2\left|\nabla_{y} u(t, x)\right|^{2} .
$$

Thus we get

$$
4 \sum_{j, k=1}^{N-1} \operatorname{Re} \int \partial_{j k}^{2} \psi_{R}(y) \partial_{j} \bar{u}(t, x) \partial_{k} u(t, x) d x-8\left\|\nabla_{y} u(t)\right\|_{L^{2}}^{2} \leq 0 .
$$

By the conservation of mass and the fact $\left\|\Delta_{y} \psi_{R}\right\|_{L^{\infty}} \lesssim R^{-2}$, we have

$$
\left.\left|\int \Delta_{y}^{2} \psi_{R}(y)\right| u(t, x)\right|^{2} d x \mid \lesssim R^{-2}
$$

Moreover, since $\psi_{R}(y)=|y|^{2}$ for $|y| \leq R$ and $\left\|\Delta_{y} \psi_{R}\right\|_{L^{\infty}} \lesssim 1$, we see that

$$
\left.\left.\left|\int\left(2(N-1)-\Delta_{y} \psi_{R}(y)\right)\right| x\right|^{-b}|u(t, x)|^{\alpha+2} d x\left|\lesssim \int_{|y| \geq R}\right| x\right|^{-b}|u(t, x)|^{\alpha+2} d x .
$$

Similarly, we have

$$
\left.\left.\left|\int\left(2|x|^{2}-\frac{\psi_{R}^{\prime}(\tau)}{\tau}|y|^{2}-2 x_{N}^{2}\right)\right| x\right|^{-b-2}|u(t, x)|^{\alpha+2} d x\left|\lesssim \int_{|y| \geq R}\right| x\right|^{-b}|u(t, x)|^{\alpha+2} d x .
$$

We thus obtain

$$
V_{\varphi_{R}}^{\prime \prime}(t) \leq 8 G(u(t))+C R^{-2}+C R^{-b} \int_{|y| \geq R}|u(t, x)|^{\alpha+2} d x
$$


To estimate the last term in the right hand side of (4.10), we recall the following radial Sobolev embedding due to Strauss [44]: for any radial function $f: \mathbb{R}^{N-1} \rightarrow \mathbb{C}$, it holds that

$$
\sup _{y \neq 0}|y|^{\frac{N-2}{2}}|f(y)| \leq C(N)\|f\|_{L_{y}^{2}}^{\frac{1}{2}}\left\|\nabla_{y} f\right\|_{L_{y}^{2}}^{\frac{1}{2}}
$$

We estimate

$$
\int_{\mathbb{R}} \int_{|y| \geq R}\left|u\left(t, y, x_{N}\right)\right|^{\alpha+2} d y d x_{N} \leq \int_{\mathbb{R}}\left\|u\left(t, x_{N}\right)\right\|_{L_{y}^{\infty}(|y| \geq R)}^{\alpha}\left\|u\left(t, x_{N}\right)\right\|_{L_{y}^{2}}^{2} d x_{N} .
$$

We consider separately two subcases: $\alpha=2$ and $\alpha<2$.

Subcase 1. $\alpha=2$. We have

$$
\int_{\mathbb{R}} \int_{|y| \geq R}\left|u\left(t, y, x_{N}\right)\right|^{\alpha+2} d y d x_{N} \leq\left(\sup _{x_{N} \in \mathbb{R}}\left\|u\left(t, x_{N}\right)\right\|_{L_{y}^{2}}^{2}\right) \int_{\mathbb{R}}\left\|u\left(t, x_{N}\right)\right\|_{L_{y}^{\infty}(|y| \geq R)}^{2} d x_{N} .
$$

By the radial Sobolev embedding (4.11) and the conservation of mass, we have

$$
\begin{aligned}
\int_{\mathbb{R}}\left\|u\left(t, x_{N}\right)\right\|_{L_{y}^{\infty}(|y| \geq R)}^{2} d x_{N} & \lesssim R^{-\frac{N-2}{2}} \int_{\mathbb{R}}\left\|u\left(t, x_{N}\right)\right\|_{L_{y}^{2}}\left\|\nabla_{y} u\left(t, x_{N}\right)\right\|_{L_{y}^{2}} d x_{N} \\
& \lesssim R^{-\frac{N-2}{2}}\left(\int_{\mathbb{R}}\left\|u\left(t, x_{N}\right)\right\|_{L_{y}^{2}}^{2} d x_{N}\right)^{1 / 2}\left(\int_{\mathbb{R}}\left\|\nabla_{y} u\left(t, x_{N}\right)\right\|_{L_{y}^{2}}^{2} d x_{N}\right)^{1 / 2} \\
& =R^{-\frac{N-2}{2}}\|u(t)\|_{L_{x}^{2}}\left\|\nabla_{y} u(t)\right\|_{L_{x}^{2}} \\
& \lesssim R^{-\frac{N-2}{2}}\left\|\nabla_{y} u(t)\right\|_{L_{x}^{2}} .
\end{aligned}
$$

Set $g\left(x_{N}\right):=\left\|u\left(t, x_{N}\right)\right\|_{L_{y}^{2}}^{2}$. We have

$$
g\left(x_{N}\right)=\int_{-\infty}^{x_{N}} \partial_{s} g(s) d s=2 \int_{-\infty}^{x_{N}} \operatorname{Re} \int_{\mathbb{R}^{N-1}} \bar{u}(t, y, s) \partial_{s} u(t, y, s) d y d s \leq 2\|u(t)\|_{L_{x}^{2}}\left\|\partial_{N} u(t)\right\|_{L_{x}^{2}} .
$$

Thus we get

$$
\sup _{x_{N} \in \mathbb{R}}\left\|u\left(t, x_{N}\right)\right\|_{L_{y}^{2}}^{2} \leq C\left\|\partial_{N} u(t)\right\|_{L_{x}^{2}}
$$

This shows that

$$
\int_{\mathbb{R}} \int_{|y| \geq R}\left|u\left(t, y, x_{N}\right)\right|^{\alpha+2} d y d x_{N} \lesssim R^{-\frac{N-2}{2}}\left\|\nabla_{y} u(t)\right\|_{L_{x}^{2}}\left\|\partial_{N} u(t)\right\|_{L_{x}^{2}} \lesssim R^{-\frac{N-2}{2}}\|\nabla u(t)\|_{L_{x}^{2}}^{2} .
$$

Subcase 2. $\alpha<2$. We have

$$
\int_{\mathbb{R}} \int_{|y| \geq R}\left|u\left(t, y, x_{N}\right)\right|^{\alpha+2} d y d x_{N} \leq\left(\int_{\mathbb{R}}\left\|u\left(t, x_{N}\right)\right\|_{L_{y}^{\infty}(|y| \geq R)}^{2} d x_{N}\right)^{\frac{\alpha}{2}}\left(\int_{\mathbb{R}}\left\|u\left(t, x_{N}\right)\right\|_{L_{y}^{2}}^{\frac{4}{2-\alpha}} d x_{N}\right)^{\frac{2-\alpha}{2}} .
$$

By the Gagliardo-Nirenberg inequality, we have

$$
\int_{\mathbb{R}}\left\|u\left(t, x_{N}\right)\right\|_{L_{y}^{2}}^{\frac{4}{2-\alpha}} d x_{N} \lesssim\left\|\partial_{N}\left(\left\|u\left(t, x_{N}\right)\right\|_{L_{y}^{2}}\right)\right\|_{L_{x_{N}}^{2}}^{\frac{\alpha}{2-\alpha}}\|\| u\left(t, x_{N}\right)\left\|_{L_{y}^{2}}\right\|_{L_{x_{N}}^{2}}^{\frac{4-\alpha}{2-\alpha}} .
$$

By the Cauchy-Schwarz inequality, we see that

$$
\begin{aligned}
2\left|\partial_{N}\left(\left\|u\left(t, x_{N}\right)\right\|_{L_{y}^{2}}\right)\right|\left\|u\left(t, x_{N}\right)\right\|_{L_{y}^{2}} & =\left|\partial_{N}\left(\left\|u\left(t, x_{N}\right)\right\|_{L_{y}^{2}}^{2}\right)\right| \\
& =2\left|\operatorname{Re} \int_{\mathbb{R}^{N-1}} \bar{u}\left(t, y, x_{N}\right) \partial_{N} u\left(t, y, x_{N}\right) d y\right| \\
& \leq 2\left\|u\left(t, x_{N}\right)\right\|_{L_{y}^{2}}\left\|\partial_{N} u\left(t, x_{N}\right)\right\|_{L_{y}^{2}}
\end{aligned}
$$

which implies that $\left|\partial_{N}\left(\left\|u\left(t, x_{N}\right)\right\|_{L_{y}^{2}}\right)\right| \leq\left\|\partial_{N} u\left(t, x_{N}\right)\right\|_{L_{y}^{2}}$. It follows that

$$
\begin{aligned}
\int_{\mathbb{R}}\left\|u\left(t, x_{N}\right)\right\|_{L_{y}^{2}}^{\frac{4}{2-\alpha}} d x_{N} & \lesssim\|\| \partial_{N} u\left(t, x_{N}\right)\left\|_{L_{y}^{2}}\right\|_{L_{x_{N}}^{2}}^{\frac{\alpha}{2-\alpha}}\|u(t)\|_{L_{x}^{2}}^{\frac{4-\alpha}{2-\alpha}} \\
& =\left\|\partial_{N} u(t)\right\|_{L_{x}^{2}}^{\frac{\alpha}{2-\alpha}}\|u(t)\|_{L_{x}^{2}}^{\frac{4-\alpha}{2-\alpha}} \\
& \lesssim\left\|\partial_{N} u(t)\right\|_{L_{x}^{2}}^{\frac{\alpha}{2-\alpha}}
\end{aligned}
$$


Thus, by the Young inequality, we get

$$
\begin{aligned}
\int_{\mathbb{R}} \int_{|y| \geq R}\left|u\left(t, y, x_{N}\right)\right|^{\alpha+2} d y d x_{N} & \lesssim R^{-\frac{(N-2) \alpha}{4}}\left\|\nabla_{y} u(t)\right\|_{L_{x}^{2}}^{\frac{\alpha}{2}}\left\|\partial_{N} u(t)\right\|_{L_{x}^{2}}^{\frac{\alpha}{2}} \\
& \lesssim R^{-\frac{(N-2) \alpha}{4}}\left(\left\|\nabla_{y} u(t)\right\|_{L_{x}^{2}}\left\|\partial_{N} u(t)\right\|_{L_{x}^{2}}+1\right) \\
& \lesssim R^{-\frac{(N-2) \alpha}{4}}\|\nabla u(t)\|_{L_{x}^{2}}^{2}+C R^{-\frac{(N-2) \alpha}{4}} .
\end{aligned}
$$

Collecting the above subcases and using (4.10), we obtain

$$
V_{\varphi_{R}}^{\prime \prime}(t) \leq 8 G(u(t))+C R^{-2}+ \begin{cases}C R^{-\frac{N-2}{2}-b}\|\nabla u(t)\|_{L^{2}}^{2} & \text { if } \quad \alpha=2, \\ C R^{-\frac{(N-2) \alpha}{4}-b}\|\nabla u(t)\|_{L^{2}}^{2}+C R^{-\frac{(N-2) \alpha}{4}-b} & \text { if } \quad \alpha<2,\end{cases}
$$

for all $t \in\left(-T_{*}, T^{*}\right)$. Under the assumptions (1.7) and (1.10), we have the following estimate due to $[11,(5.8)]$ : for $\varepsilon>0$ small enough, there exists a constant $\delta=\delta(\varepsilon)>0$ such that

$$
8 G(u(t))+\varepsilon\|\nabla u(t)\|_{L^{2}}^{2} \leq-\delta
$$

for all $t \in\left(-T_{*}, T^{*}\right)$. Thanks to (4.12), we take $R>1$ sufficiently large to get

$$
V_{\psi_{R}}^{\prime \prime}(t) \leq-\frac{\delta}{2}<0
$$

for all $t \in\left(-T_{*}, T^{*}\right)$. The standard convexity argument yields $T_{*}, T^{*}<\infty$. The proof is complete.

We are next interested in long time dynamics of $H^{1}$-solutions for (1.2) with data at the ground state threshold. To this end, we need the following lemmas.

Lemma 4.1. Let $N \geq 1,0<b<\min \{2, N\}$, and $0<\alpha<\alpha(N)$. Let $\left(f_{n}\right)_{n>1}$ be a bounded sequence in $H^{1}$. Then, there exist a subsequence still denoted by $\left(f_{n}\right)_{n \geq 1}$ and a function $f \in H^{1}$ such that:

- $f_{n} \rightarrow f$ weakly in $H^{1}$.

- $f_{n} \rightarrow f$ strongly in $L_{\text {loc }}^{r}$ for all $1 \leq r<2^{*}$.

- $\lim _{n \rightarrow \infty} P\left(f_{n}\right)=P(f)$ as $n \rightarrow \infty$, where $P$ is as in (1.12).

Proof. The first two items are well-known. Let us prove the last one. Let $\varepsilon>0$. Since $\left(f_{n}\right)_{n \geq 1}$ is bounded in $H^{1}$, we have for any $R>0$,

$$
\begin{aligned}
\left.\left|\int_{|x| \geq R}\right| x\right|^{-b}\left(\left|f_{n}(x)\right|^{\alpha+2}-|f(x)|^{\alpha+2}\right) d x \mid & \leq R^{-b}\left(\left\|f_{n}\right\|_{L^{\alpha+2}}^{\alpha+2}+\|f\|_{L^{\alpha+2}}^{\alpha+2}\right) \\
& \leq C R^{-b}\left(\left\|f_{n}\right\|_{H^{1}}^{\alpha+2}+\|f\|_{H^{1}}^{\alpha+2}\right) \\
& \leq C R^{-b} .
\end{aligned}
$$

By choosing $R>0$ sufficiently large, we have

$$
\left.\left|\int_{|x| \geq R}\right| x\right|^{-b}\left(\left|f_{n}(x)\right|^{\alpha+2}-|f(x)|^{\alpha+2}\right) d x \mid<\frac{\varepsilon}{2} .
$$

On the other hand, we have

$$
\left.\left|\int_{|x| \leq R}\right| x\right|^{-b}\left(\left|f_{n}(x)\right|^{\alpha+2}-|f(x)|^{\alpha+2}\right) d x\left|\leq\left\||x|^{-b}\right\|_{L^{\delta}(|x| \leq R)}\left\|\left|f_{n}\right|^{\alpha+2}-|f|^{\alpha+2}\right\|_{L^{\mu}(|x| \leq R)}\right.
$$

provided that $\delta, \mu \geq 1,1=\frac{1}{\delta}+\frac{1}{\mu}$. The term $\left\||x|^{-b}\right\|_{L^{\delta}(|x| \leq R)}$ is finite provided that $\frac{N}{\delta}>b$. Thus $\frac{1}{\delta}>\frac{b}{N}$ and $\frac{1}{\mu}=1-\frac{1}{\delta}<\frac{N-b}{N}$. We next bound

$$
\left\|\left|f_{n}\right|^{\alpha+2}-|f|^{\alpha+2}\right\|_{L^{\mu}(|x| \leq R)} \lesssim\left(\left\|f_{n}\right\|_{L^{\sigma}}^{\alpha+1}+\|f\|_{L^{\sigma}}^{\alpha+1}\right)\left\|f_{n}-f\right\|_{L^{\sigma}(|x| \leq R)}
$$

provided that

$$
\frac{\alpha+2}{\sigma}=\frac{1}{\mu}<\frac{N-b}{N}
$$

By the Sobolev embedding $H^{1} \hookrightarrow L^{r}$ for any $2 \leq r<2^{*}$ and the fact that $f_{n} \rightarrow f$ strongly in $L^{r}(|x| \leq R)$ for any $1 \leq r<2^{*}$, we are able to choose $\sigma \in\left(2,2^{*}\right)$ so that (4.15) holds. Indeed, in the case $N \geq 3$, we choose $\sigma$ smaller but close to $\frac{2 N}{N-2}$. We see that (4.15) is satisfied provided that

$$
\frac{(\alpha+2)(N-2)}{2 N}<\frac{N-b}{N} .
$$


This condition is fulfilled since $\alpha<\frac{4-2 b}{N-2}$. In the case $N=1,2$, we see that $(4.15)$ is satisfied by choosing $\sigma$ sufficiently large. As a consequence, we get

$$
\left.\left|\int_{|x| \leq R}\right| x\right|^{-b}\left(\left|f_{n}(x)\right|^{\alpha+2}-|f(x)|^{\alpha+2}\right) d x \mid \leq C\left\|f_{n}-f\right\|_{L^{\sigma}(|x| \leq R)}<\frac{\varepsilon}{2}
$$

for $n$ sufficiently large. Collecting (4.14) and (4.16), we prove the result.

Lemma 4.2. Let $N \geq 1,0<b<\min \{2, N\}$, and $0<\alpha<\alpha(N)$. Let $Q$ be the unique positive radial solution to (1.9). Let $\left(f_{n}\right)_{n \geq 1}$ be a sequence of $H^{1}$-functions satisfying

$$
M\left(f_{n}\right)=M(Q), \quad E\left(f_{n}\right)=E(Q), \quad \forall n \geq 1
$$

and

$$
\lim _{n \rightarrow \infty}\left\|\nabla f_{n}\right\|_{L^{2}}=\|\nabla Q\|_{L^{2}}
$$

Then there exists a subsequence still denoted by $\left(f_{n}\right)_{n \geq 1}$ such that

$$
f_{n} \rightarrow e^{i \theta} Q \quad \text { strongly in } H^{1}
$$

for some $\theta \in \mathbb{R}$ as $n \rightarrow \infty$.

Proof. Since $\left(f_{n}\right)$ is a bounded sequence in $H^{1}$, by Lemma 4.1, there exist a subsequence still denoted by $\left(f_{n}\right)_{n \geq 1}$ and a function $f \in H^{1}$ such that $f_{n} \rightarrow f$ weakly in $H^{1}$ and $P\left(f_{n}\right) \rightarrow P(f)$ as $n \rightarrow \infty$. We first observe that

$$
\begin{aligned}
P(f)=\lim _{n \rightarrow \infty} P\left(f_{n}\right) & =\lim _{n \rightarrow \infty}(\alpha+2)\left(\frac{1}{2}\left\|\nabla f_{n}\right\|_{L^{2}}^{2}-E\left(f_{n}\right)\right) \\
& =(\alpha+2)\left(\frac{1}{2}\|\nabla Q\|_{L^{2}}^{2}-E(Q)\right) \\
& =\frac{2(\alpha+2)}{N \alpha+2 b}\|\nabla Q\|_{L^{2}}^{2}=P(Q) .
\end{aligned}
$$

This shows that $f \neq 0$. Moreover, by the Gagliardo-Nirenberg inequality (2.3), we have

$$
P(f)-C_{\mathrm{opt}}\|\nabla f\|_{L^{2}}^{\frac{N \alpha+2 b}{2}}\|f\|_{L^{2}}^{\frac{4-2 b-(N-2) \alpha}{2}} \leq 0 .
$$

By the lower continuity of weak convergence, we have

$$
\|\nabla f\|_{L^{2}} \leq \liminf _{n \rightarrow \infty}\left\|\nabla f_{n}\right\|_{L^{2}}^{2}
$$

which implies that

$$
\begin{aligned}
P(f)-C_{\mathrm{opt}}\|\nabla f\|_{L^{2}}^{\frac{N \alpha+2 b}{2}}\|f\|_{L^{2}}^{\frac{4-2 b-(N-2) \alpha}{2}} & \geq \liminf _{n \rightarrow \infty} P\left(f_{n}\right)-C_{\mathrm{opt}}\left\|\nabla f_{n}\right\|_{L^{2}}^{\frac{N \alpha+2 b}{2}}\left\|f_{n}\right\|_{L^{2}}^{\frac{4-2 b-(N-2) \alpha}{2}} \\
& =P(Q)-C_{\mathrm{opt}}\|\nabla Q\|_{L^{2}}^{\frac{N \alpha+2 b}{2}}\|Q\|_{L^{2}}^{\frac{4-2 b-(N-2) \alpha}{2}}=0 .
\end{aligned}
$$

This shows that $f$ is an optimizer for the Gagliardo-Nirenberg inequality (2.3). We also have

$$
\|\nabla f\|_{L^{2}}=\lim _{n \rightarrow \infty}\left\|\nabla f_{n}\right\|_{L^{2}}^{2}
$$

hence $f_{n} \rightarrow f$ strongly in $H^{1}$. We claim that there exists $\theta \in \mathbb{R}$ such that $f(x)=e^{i \theta} g(x)$, where $g$ is a non-negative radial optimizer for (2.3). Indeed, since $\|\nabla(|f|)\|_{L^{2}} \leq\|\nabla f\|_{L^{2}}$, it is clear that $|f|$ is also an optimizer for (2.3) and

$$
\|\nabla(|f|)\|_{L^{2}}=\|\nabla f\|_{L^{2}}
$$

Set $w(x):=\frac{f(x)}{|f(x)|}$. Since $|w(x)|^{2}=1$, it follows that $\operatorname{Re}(\bar{w} \nabla w(x))=0$ and

$$
\nabla f(x)=\nabla(|f(x)|) w(x)+|f(x)| \nabla w(x)=w(x)(\nabla(|f(x)|)+|f(x)| \bar{w}(x) \nabla w(x))
$$

which implies $|\nabla f(x)|^{2}=|\nabla(|f(x)|)|^{2}+|f(x)|^{2}|\nabla w(x)|^{2}$ for all $x \in \mathbb{R}^{3}$. From (4.17), we get

$$
\int_{\mathbb{R}^{3}}|f(x)|^{2}|\nabla w(x)|^{2} d x=0
$$

which shows $|\nabla w(x)|=0$, hence $w(x)$ is a constant, and the claim follows with $g(x)=|f(x)|$. Moreover, by replacing $g$ with its symmetric rearrangement, we can assume that $g$ is radially symmetric. Since $g$ is an optimizer for (2.3), $g$ must satisfy the Euler-Lagrange equation

$$
\left.\frac{d}{d \varepsilon}\right|_{\varepsilon=0} W(g+\varepsilon \phi)=0
$$


where $W$ is the Weinstein functional

$$
W(f):=P(f) \div\left[\|\nabla f\|_{L^{2}}^{\frac{N \alpha+2 b}{2}}\|f\|_{L^{2}}^{\frac{4-2 b-(N-2) \alpha}{2}}\right]
$$

A direct computation shows

$$
-m \Delta g+n g-\frac{\alpha+2}{C_{\mathrm{opt}}}|x|^{-b}|g|^{\alpha} g=0
$$

where

$$
\begin{aligned}
& m:=\frac{N \alpha+2 b}{2}\|\nabla f\|_{L^{2}}^{\frac{N \alpha+2 b-4}{2}}\|f\|_{L^{2}}^{\frac{4-2 b-(N-2) \alpha}{2}}, \\
& n:=\frac{4-2 b-(N-2) \alpha}{2}\|\nabla f\|_{L^{2}}^{\frac{N \alpha+2 b}{2}}\|f\|_{L^{2}}^{-\frac{2 b+(N-2) \alpha}{2}} .
\end{aligned}
$$

By a change of variable $g(x)=\lambda \phi(\mu x)$ with $\lambda, \mu>0$ satisfying

$$
\mu^{2}=\frac{n}{m}, \quad \lambda^{\alpha}=\frac{n C_{\mathrm{opt}}}{\alpha+2} \mu^{-b}
$$

we see that $\phi$ solves (1.9) and $W(g)=W(\phi)=C_{\mathrm{opt}}$. By the uniqueness of positive radial solution to (1.9) due to $[29,48,51]$, we have $\phi \equiv Q$. As $\|g\|_{L^{2}}=\|Q\|_{L^{2}}$ and $\|\nabla g\|_{L^{2}}=\|\nabla Q\|_{L^{2}}$, we infer that $\lambda=\mu=1$. This shows that $f(x)=e^{i \theta} Q(x)$ for some $\theta \in \mathbb{R}$. The proof is complete.

Proof of Theorem 1.4. We consider separately three cases.

Case 1. Let $u_{0} \in H^{1}$ satisfy (1.19) and (1.20). We first note that (1.19) and (1.20) are invariant under the scaling

$$
u_{0}^{\lambda}(x):=\lambda^{\frac{2-b}{\alpha}} u_{0}(\lambda x), \quad \lambda>0 .
$$

By choosing a suitable scaling, we can assume that

$$
M\left(u_{0}\right)=M(Q), \quad E\left(u_{0}\right)=E(Q) .
$$

Thus (1.20) becomes $\left\|\nabla u_{0}\right\|_{L^{2}}<\|\nabla Q\|_{L^{2}}$. We first claim that

$$
\|\nabla u(t)\|_{L^{2}}<\|\nabla Q\|_{L^{2}}
$$

for all $t \in\left(-T_{*}, T^{*}\right)$. Assume by contradiction that there exists $t_{0} \in\left(-T_{*}, T^{*}\right)$ such that $\left\|\nabla u\left(t_{0}\right)\right\|_{L^{2}} \geq$ $\|\nabla Q\|_{L^{2}}$. By continuity, there exists $t_{1} \in\left(-T_{*}, T^{*}\right)$ such that $\left\|\nabla u\left(t_{1}\right)\right\|_{L^{2}}=\|\nabla Q\|_{L^{2}}$. By the conservation of energy and (2.6), we see that

$$
\begin{aligned}
P\left(u\left(t_{1}\right)\right) & =(\alpha+2)\left(\frac{1}{2}\left\|\nabla u\left(t_{1}\right)\right\|_{L^{2}}^{2}-E\left(u\left(t_{1}\right)\right)\right) \\
& =(\alpha+2)\left(\frac{1}{2}\|\nabla Q\|_{L^{2}}^{2}-E(Q)\right) \\
& =\frac{2(\alpha+2)}{N \alpha+2 b}\|\nabla Q\|_{L^{2}}^{2} .
\end{aligned}
$$

This shows that $u\left(t_{1}\right)$ is an optimizer for the Gagliardo-Nirenberg inequality (2.3). Arguing as in the proof of Lemma 4.2, we have $u\left(t_{1}\right)=e^{i \theta} Q$ for some $\theta \in \mathbb{R}$. Moreover, by the uniqueness of solution to (1.2), we infer that $u(t)=e^{i t} e^{i \theta} Q$ which contradicts (1.20). This shows (4.20). In particular, the solution exists globally in time. We now have two possibilities.

First possibility. If

then there exists $\rho>0$ such that

$$
\sup _{t \in \mathbb{R}}\|\nabla u(t)\|_{L^{2}}<\|\nabla Q\|_{L^{2}}
$$

$$
\|\nabla u(t)\|_{L^{2}} \leq(1-\rho)\|\nabla Q\|_{L^{2}}
$$

which, by (4.19), implies that (4.1) holds for all $t \in \mathbb{R}$. By the same argument as in the proof of Theorem 1.3 , we prove (1.21). In particular, if $N \geq 2$ and $0<b<\min \left\{2, \frac{N}{2}\right\}$, then by Theorem 1.1, the solution scatters in $H^{1}$ in both directions.

\section{Second possibility. If}

$$
\sup _{t \in \mathbb{R}}\|\nabla u(t)\|_{L^{2}}=\|\nabla Q\|_{L^{2}}
$$

then there exists a time sequence $\left(t_{n}\right)_{n \geq 1} \subset \mathbb{R}$ such that

$$
M\left(u\left(t_{n}\right)\right)=M(Q), \quad E\left(u\left(t_{n}\right)\right)=E(Q), \quad \lim _{n \rightarrow \infty}\left\|\nabla u\left(t_{n}\right)\right\|_{L^{2}}=\|\nabla Q\|_{L^{2}} .
$$


We notice that $\left|t_{n}\right| \rightarrow \infty$. Otherwise, passing to a subsequence if necessary, we have $t_{n} \rightarrow t_{0}$ as $n \rightarrow \infty$. By continuity of the solution, we have $u\left(t_{n}\right) \rightarrow u\left(t_{0}\right)$ strongly in $H^{1}$. This implies that $u\left(t_{0}\right)$ is an optimizer for (2.3) which is a contradiction.

Applying Lemma 4.2 with $f_{n}=u\left(t_{n}\right)$, we prove that up to a subsequence,

$$
u\left(t_{n}\right) \rightarrow e^{i \theta} Q \text { strongly in } H^{1}
$$

for some $\theta \in \mathbb{R}$ as $n \rightarrow \infty$.

Case 2. Let $u_{0} \in H^{1}$ satisfy (1.19) and (1.23). By the scaling (4.18), we can assume that

$$
M\left(u_{0}\right)=M(Q), \quad\left\|\nabla u_{0}\right\|_{L^{2}}=\|\nabla Q\|_{L^{2}}, \quad E\left(u_{0}\right)=E(Q) .
$$

In particular, $u_{0}$ is an optimizer for (2.3) which implies $u_{0}(x)=e^{i \theta} Q(x)$ for some $\theta \in \mathbb{R}$. By the uniqueness of solution to (1.2), we have $u(t, x)=e^{i t} e^{i \theta} Q(x)$.

Case 3. Let $u_{0} \in H^{1}$ satisfy (1.19) and (1.24). As in Case 1, we can assume that

$$
M\left(u_{0}\right)=M(Q), \quad E\left(u_{0}\right)=E(Q), \quad\left\|\nabla u_{0}\right\|_{L^{2}}>\|\nabla Q\|_{L^{2}} .
$$

Arguing as above, we prove that

$$
\|\nabla u(t)\|_{L^{2}}>\|\nabla Q\|_{L^{2}}
$$

for all $t \in\left(-T_{*}, T^{*}\right)$. Let us consider only positive times. The one for negative times is similar. If $T^{*}<\infty$, then we are done. Otherwise, if $T^{*}=\infty$, then we consider two possibilities.

First possibility. If

$$
\sup _{t \in[0, \infty)}\|\nabla u(t)\|_{L^{2}}>\|\nabla Q\|_{L^{2}}
$$

then there exists $\rho>0$ such that

$$
\|\nabla u(t)\|_{L^{2}} \geq(1+\rho)\|\nabla Q\|_{L^{2}}
$$

for all $t \in[0, \infty)$. By (4.21) and the conservation laws of mass and energy, we have

$$
\begin{aligned}
G(u(t))[M(u(t))]^{\sigma_{c}} & =\frac{N \alpha+2 b}{2} E(u(t))[M(u(t))]^{\sigma_{c}}-\frac{N \alpha-4+2 b}{4}\left(\|\nabla u(t)\|_{L^{2}}\|u(t)\|_{L^{2}}^{\sigma_{c}}\right)^{2} \\
& \leq \frac{N \alpha+2 b}{2} E(Q)[M(Q)]^{\sigma_{c}}-\frac{N \alpha-4+2 b}{4}\left((1+\rho)\|\nabla Q\|_{L^{2}}\|Q\|_{L^{2}}^{\sigma_{c}}\right)^{2} \\
& =-\frac{N \alpha-4+2 b}{4}\left((1+\rho)^{2}-1\right)\left(\|\nabla Q\|_{L^{2}}\|Q\|_{L^{2}}^{\sigma_{c}}\right)^{2}
\end{aligned}
$$

for all $t \in[0, \infty)$. By Theorem 1.2, there exists a time sequence $t_{n} \rightarrow \infty$ such that $\left\|\nabla u\left(t_{n}\right)\right\|_{L^{2}} \rightarrow \infty$ as $n \rightarrow \infty$.

\section{Second possibility. If}

$$
\sup _{t \in[0, \infty)}\|\nabla u(t)\|_{L^{2}}=\|\nabla Q\|_{L^{2}}
$$

then there exists a time sequence $\left(t_{n}\right)_{n \geq 1}$ such that $\left\|\nabla u\left(t_{n}\right)\right\|_{L^{2}} \rightarrow\|\nabla Q\|_{L^{2}}$ as $n \rightarrow \infty$. Arguing as in Case 1 , we show that $t_{n} \rightarrow \infty$ and

$$
u\left(t_{n}\right) \rightarrow e^{i \theta} Q \text { strongly in } H^{1}
$$

for some $\theta \in \mathbb{R}$ as $n \rightarrow \infty$. This completes the first part of Item (3) of Theorem 1.4.

Let us prove the second part of Item (3) of Theorem 1.4.

- Finite variance data. If we assume in addition that $u_{0} \in \Sigma$, then the first possibility cannot occur. In fact, if it occurs, then there exists $\delta>0$ such that

$$
G(u(t)) \leq-\delta
$$

for all $t \in[0, \infty)$. This is impossible by the convexity argument as

$$
\frac{d^{2}}{d t^{2}}\|x u(t)\|_{L^{2}}^{2}=8 G(u(t))
$$

- Radially symmetric data. If we assume in addition that $N \geq 2, \alpha \leq 4$, and $u_{0}$ is radially symmetric, then the first possibility cannot occur. In fact, suppose that the first possibility occurs, so (4.22) holds. 
It follows from (4.21) and (2.7) that

$$
\begin{aligned}
8 G(u(t)) & +\varepsilon\|\nabla u(t)\|_{L^{2}}^{2} \\
& =4(N \alpha+2 b) E(u(t))[M(u(t))]^{\sigma_{c}}-(2 N \alpha-4 b+8-\varepsilon)\|\nabla u(t)\|_{L^{2}}^{2}[M(u(t))]^{\sigma_{c}} \\
& \leq 4(N \alpha+2 b) E(Q)[M(Q)]^{\sigma_{c}}-(2 N \alpha-4 b+8-\varepsilon)(1+\rho)^{2}\left(\|\nabla Q\|_{L^{2}}\|Q\|_{L^{2}}^{\sigma_{c}}\right)^{2} \\
& =-2(N \alpha-4+2 b)\left(\|\nabla Q\|_{L^{2}}\|Q\|_{L^{2}}^{\sigma_{c}}\right)^{2}(1+\rho)^{2}\left[\frac{(1+\rho)^{2}-1}{(1+\rho)^{2}}-\frac{\varepsilon}{2(N \alpha-4+2 b)}\right]
\end{aligned}
$$

for all $t \in[0, \infty)$. Taking $\varepsilon>0$ sufficiently small, there exists $\delta=\delta(\varepsilon)>0$ such that

$$
8 G(u(t))+\varepsilon\|\nabla u(t)\|_{L^{2}}^{2} \leq-\delta
$$

for all $t \in[0, \infty)$. We recall the following estimate due to [11, Lemma 3.4]: for any $R>1$ and any $\varepsilon>0$,

$$
V_{\varphi_{R}}^{\prime \prime}(t) \leq 8 G(u(t))+ \begin{cases}C R^{-2}+C R^{-[2(N-1)+b]}\|\nabla u(t)\|_{L^{2}}^{2} & \text { if } \quad \alpha=4, \\ C R^{-2}+C \varepsilon^{-\frac{\alpha}{4-\alpha}} R^{-\frac{2[(N-1) \alpha+2 b]}{4-\alpha}}+\varepsilon\|\nabla u(t)\|_{L^{2}}^{2} & \text { if } \quad \alpha<4 .\end{cases}
$$

Thanks to (4.23), we take $R>1$ sufficiently large if $\alpha=4$, and $\varepsilon>0$ sufficiently small and $R>1$ sufficiently large depending on $\varepsilon$, we obtain

$$
V_{\varphi_{R}}^{\prime \prime}(t) \leq-\frac{\delta}{2}
$$

for all $t \in[0, \infty)$. This is impossible.

- Cylindrically symmetric data. If we assume in addition that $N \geq 3, \alpha \leq 2$, and $u_{0} \in \Sigma_{N}$, then the first possibility cannot occur. This is done by the same argument as above using (4.12) and (4.23). The proof of Theorem 1.4 is now complete.

Finally, we study long time dynamics of $H^{1}$-solutions for (1.2) with data above the ground state threshold.

Proof of Theorem 1.5. Let us consider two cases.

Case 1. Let $u_{0} \in \Sigma$ satisfy (1.27), (1.28), (1.29), and (1.30). We will show that (1.11) holds. To this end, let us start with the following estimate: for $f \in \Sigma$,

$$
\left(\operatorname{Im} \int \bar{f} x \cdot \nabla f d x\right)^{2} \leq\|x f\|_{L^{2}}^{2}\left(\|\nabla f\|_{L^{2}}^{2}-\left[C_{\text {opt }}\right]^{-\frac{4}{N \alpha+2 b}}[M(f)]^{-\frac{4-2 b-(N-2) \alpha}{N \alpha+2 b}}[P(f)]^{\frac{4}{N \alpha+2 b}}\right) .
$$

In fact, let $\lambda>0$. We have

$$
\int\left|\nabla\left(e^{i \lambda|x|^{2}} f\right)\right|^{2} d x=4 \lambda^{2}\|x f\|_{L^{2}}^{2}+4 \lambda \operatorname{Im} \int \bar{f} x \cdot \nabla f d x+\|\nabla f\|_{L^{2}}^{2}
$$

By the Gagliardo-Nirenberg inequality (2.3), we have

$$
[P(f)]^{\frac{4}{N \alpha+2 b}}=\left[P\left(e^{i \lambda|x|^{2}} f\right)\right]^{\frac{4}{N \alpha+2 b}} \leq\left[C_{\mathrm{opt}}\right]^{\frac{4}{N \alpha+2 b}}\left\|\nabla\left(e^{i \lambda|x|^{2}} f\right)\right\|_{L^{2}}^{2}\|f\|_{L^{2}}^{\frac{2[4-2 b-(N-2) \alpha]}{N \alpha+2 b}}
$$

or

$$
\left\|\nabla\left(e^{i \lambda|x|^{2}} f\right)\right\|_{L^{2}}^{2} \geq\left[C_{\text {opt }}\right]^{-\frac{4}{N \alpha+2 b}} M(f)^{-\frac{4-2 b-(N-2) \alpha}{N \alpha+2 b}}[P(f)]^{\frac{4}{N \alpha+2 b}} .
$$

It follows that

$$
4 \lambda^{2}\|x f\|_{L^{2}}^{2}+4 \lambda \operatorname{Im} \int \bar{f} x \cdot \nabla f d x+\|\nabla f\|_{L^{2}}^{2}-\left[C_{\mathrm{opt}}\right]^{-\frac{4}{N \alpha+2 b}}[M(f)]^{-\frac{4-2 b-(N-2) \alpha}{N \alpha+2 b}}[P(f)] \frac{4}{\frac{4}{N \alpha+2 b}} \geq 0
$$

for all $\lambda>0$. Since the left hand side is a quadratic polynomial in $\lambda$, its discriminant must be non-positive which proves (4.24).

We also have

$$
\begin{aligned}
V^{\prime \prime}(t) & =8\|\nabla u(t)\|_{L^{2}}^{2}-\frac{4(N \alpha+2 b)}{\alpha+2} P(u(t)) \\
& =16 E(u(t))-\frac{4(N \alpha-4+2 b)}{\alpha+2} P(u(t)) \\
& =4(N \alpha+2 b) E(u(t))-2(N \alpha-4+2 b)\|\nabla u(t)\|_{L^{2}}^{2}
\end{aligned}
$$

which implies that

$$
\begin{aligned}
P(u(t)) & =\frac{\alpha+2}{4(N \alpha-4+2 b)}\left(16 E(u(t))-V^{\prime \prime}(t)\right), \\
\|\nabla u(t)\|_{L^{2}}^{2} & =\frac{1}{2(N \alpha-4+2 b)}\left(4(N \alpha+2 b) E(u(t))-V^{\prime \prime}(t)\right) .
\end{aligned}
$$


Since $P(u(t)) \geq 0$, we have $V^{\prime \prime}(t) \leq 16 E(u(t))=16 E\left(u_{0}\right)$. Inserting the above identities to (4.24), we get

$$
\begin{aligned}
\left(V^{\prime}(t)\right)^{2} \leq 16 V(t) & {\left[\frac{1}{2(N \alpha-4+2 b)}\left(4(N \alpha+2 b) E(u(t))-V^{\prime \prime}(t)\right)\right.} \\
- & {\left.\left[C_{\mathrm{opt}}\right]^{-\frac{4}{N \alpha+2 b}}[M(u(t))]^{-\frac{4-2 b-(N-2) \alpha}{N \alpha+2 b}}\left(\frac{\alpha+2}{4(N \alpha-4+2 b)}\left(16 E(u(t))-V^{\prime \prime}(t)\right)\right)^{\frac{4}{N \alpha+2 b}}\right] }
\end{aligned}
$$

which implies

$$
\left(z^{\prime}(t)\right)^{2} \leq 4 g\left(V^{\prime \prime}(t)\right)
$$

where

$$
z(t):=\sqrt{V(t)}
$$

and

$$
\begin{aligned}
g(\lambda):=\frac{1}{2(N \alpha-4+2 b)}(4(N \alpha+2 b) E-\lambda) & \\
& \quad-\left[C_{\mathrm{opt}}\right]^{-\frac{4}{N \alpha+2 b}} M^{-\frac{4-2 b-(N-2) \alpha}{N \alpha+2 b}}\left(\frac{\alpha+2}{4(N \alpha-4+2 b)}(16 E-\lambda)\right)^{\frac{4}{N \alpha+2 b}}
\end{aligned}
$$

with $\lambda \leq 16 E$. Here we have used the notation $E(u(t))=E, M(u(t))=M$ due to the conservation of mass and energy. Since $N \alpha+2 b>4$, we see that $g(\lambda)$ is decreasing on $\left(-\infty, \lambda_{0}\right)$ and increasing on $\left(\lambda_{0}, 16 E\right)$, where $\lambda_{0}$ satisfies

$$
\frac{N \alpha+2 b}{2(\alpha+2)}=\left[C_{\mathrm{opt}}\right]^{-\frac{4}{N \alpha+2 b}} M^{-\frac{4-2 b-(N-2) \alpha}{N \alpha+2 b}}\left(\frac{\alpha+2}{4(N \alpha-4+2 b)}\left(16 E-\lambda_{0}\right)\right)^{\frac{4-N \alpha-2 b}{N \alpha+2 b}} .
$$

A direct calculation shows

$$
g\left(\lambda_{0}\right)=\frac{1}{2(N \alpha-4+2 b)}\left(4(N \alpha+2 b) E-\lambda_{0}\right)-\frac{N \alpha+2 b}{8(N \alpha-4+2 b)}\left(16 E-\lambda_{0}\right)=\frac{\lambda_{0}}{8} .
$$

Using the fact that

$$
C_{\mathrm{opt}}=\frac{2(\alpha+2)}{N \alpha+2 b}\left(\frac{2(N \alpha+2 b)}{N \alpha-4+2 b} E(Q)[M(Q)]^{\sigma_{c}}\right)^{-\frac{N \alpha-4+2 b}{4}}
$$

we infer from (4.26) that

$$
1=\frac{16 E(Q)[M(Q)]^{\sigma_{\mathrm{c}}}}{\left(16 E-\lambda_{0}\right) M^{\sigma_{\mathrm{c}}}}
$$

or

$$
\frac{E M^{\sigma_{\mathrm{c}}}}{E(Q)[M(Q)]^{\sigma_{\mathrm{c}}}}\left(1-\frac{\lambda_{0}}{16 E}\right)=1
$$

Thus the assumption (1.27) is equivalent to

$$
\lambda_{0} \geq 0
$$

Moreover, the assumption (1.28) is equivalent to

$$
\left(V^{\prime}(0)\right)^{2} \geq 2 V(0) \lambda_{0}
$$

or

$$
\left(z^{\prime}(0)\right)^{2} \geq \frac{\lambda_{0}}{2}=4 g\left(\lambda_{0}\right) .
$$

Similarly, the assumption (1.30) is equivalent to

$$
z^{\prime}(0) \geq 0
$$

Finally, the assumption (1.29) is equivalent to

$$
V^{\prime \prime}(0)>\lambda_{0}
$$


Indeed, from (1.29), we have

$$
\begin{aligned}
V^{\prime \prime}(0) & =16 E-\frac{4(N \alpha-4+2 b)}{\alpha+2} P\left(u_{0}\right) \\
& >16 E-\frac{4(N \alpha-4+2 b)}{\alpha+2} \frac{P(Q)[M(Q)]^{\sigma_{c}}}{M^{\sigma_{\mathrm{c}}}} \\
& =16\left(E-\frac{E(Q)[M(Q)]^{\sigma_{\mathrm{c}}}}{M^{\sigma_{\mathrm{c}}}}\right) \\
& =16 E\left(1-\frac{E(Q)[M(Q)]^{\sigma_{\mathrm{c}}}}{E M^{\sigma_{\mathrm{c}}}}\right) \\
& =\lambda_{0},
\end{aligned}
$$

where we have used (4.27) to get the last equality.

Next, we claim that there exists $\delta_{0}>0$ small such that for all $t \in\left[0, T^{*}\right)$,

$$
V^{\prime \prime}(t) \geq \lambda_{0}+\delta_{0}
$$

Assume (4.32) for the moment, we prove (1.11). We have

$$
\begin{aligned}
P(u(t))[M(u(t))]^{\sigma_{\mathrm{c}}} & =\frac{\alpha+2}{4(N \alpha-4+2 b)}\left(16 E-V^{\prime \prime}(t)\right) M^{\sigma_{\mathrm{c}}} \\
& \leq \frac{\alpha+2}{4(N \alpha-4+2 b)}\left(16 E-\lambda_{0}-\delta_{0}\right) M^{\sigma_{\mathrm{c}}} \\
& =\frac{4(\alpha+2)}{N \alpha-4+2 b} E(Q)[M(Q)]^{\sigma_{\mathrm{c}}}-\frac{\alpha+2}{4(N \alpha-4+2 b)} \delta_{0} M^{\sigma_{\mathrm{c}}} \\
& =(1-\rho) P(Q)[M(Q)]^{\sigma_{c}}
\end{aligned}
$$

for all $t \in\left[0, T^{*}\right)$, where $\rho:=\frac{\alpha+2}{4(N \alpha-4+2 b)} \delta_{0} \frac{M^{\sigma_{c}}}{P(Q)[M(Q)] \sigma_{c}}>0$. Here we have used (4.27) to get the third line. This shows (1.11). In particular, if $N \geq 2$ and $0<b<\min \left\{2, \frac{N}{2}\right\}$, then the solution scatters in $H^{1}$ forward in time.

It remains to show (4.32). By (4.31), we take $\delta_{1}>0$ so that

$$
V^{\prime \prime}(0) \geq \lambda_{0}+2 \delta_{1} \text {. }
$$

By continuity, we have

$$
V^{\prime \prime}(t)>\lambda_{0}+\delta_{1}, \quad \forall t \in\left[0, t_{0}\right) .
$$

for $t_{0}>0$ sufficiently small. By reducing $t_{0}$ if necessary, we can assume that

$$
z^{\prime}\left(t_{0}\right)>2 \sqrt{g\left(\lambda_{0}\right)} \text {. }
$$

In fact, if $z^{\prime}(0)>2 \sqrt{g\left(\lambda_{0}\right)}$, then (4.34) follows from the continuity argument. Otherwise, if $z^{\prime}(0)=$ $2 \sqrt{g\left(\lambda_{0}\right)}$, then using the fact that

$$
z^{\prime \prime}(t)=\frac{1}{z(t)}\left(\frac{V^{\prime \prime}(t)}{2}-\left(z^{\prime}(t)\right)^{2}\right)
$$

and (4.31), we have $z^{\prime \prime}(0)>0$. This shows (4.34) by taking $t_{0}>0$ sufficiently small. Thanks to (4.34), we take $\epsilon_{0}>0$ be a small constant so that

$$
z^{\prime}\left(t_{0}\right) \geq 2 \sqrt{g\left(\lambda_{0}\right)}+2 \epsilon_{0}
$$

We will prove by contradiction that

$$
z^{\prime}(t)>2 \sqrt{g\left(\lambda_{0}\right)}+\epsilon_{0}, \quad \forall t \geq t_{0}
$$

Suppose that it is not true and set

$$
t_{1}:=\inf \left\{t \geq t_{0}: z^{\prime}(t) \leq 2 \sqrt{g\left(\lambda_{0}\right)}+\epsilon_{0}\right\} .
$$

By (4.36), we have $t_{1}>t_{0}$. By continuity, we have

$$
z^{\prime}\left(t_{1}\right)=2 \sqrt{g\left(\lambda_{0}\right)}+\epsilon_{0}
$$

and

$$
z^{\prime}(t) \geq 2 \sqrt{g\left(\lambda_{0}\right)}+\epsilon_{0}, \quad \forall t \in\left[t_{0}, t_{1}\right]
$$


By (4.25), we see that

$$
\left(2 \sqrt{g\left(\lambda_{0}\right)}+\epsilon_{0}\right)^{2} \leq\left(z^{\prime}(t)\right)^{2} \leq 4 g\left(V^{\prime \prime}(t)\right), \quad \forall t \in\left[t_{0}, t_{1}\right]
$$

It follows that $g\left(V^{\prime \prime}(t)\right)>g\left(\lambda_{0}\right)$ for all $t \in\left[t_{0}, t_{1}\right]$, thus $V^{\prime \prime}(t) \neq \lambda_{0}$ and by continuity, $V^{\prime \prime}(t)>\lambda_{0}$ for all $t \in\left[t_{0}, t_{1}\right]$.

We will prove that there exists a constant $C>0$ such that

$$
V^{\prime \prime}(t) \geq \lambda_{0}+\frac{\sqrt{\epsilon_{0}}}{C}, \quad \forall t \in\left[t_{0}, t_{1}\right] .
$$

Indeed, by the Taylor expansion of $g$ near $\lambda_{0}$ with the fact $g^{\prime}\left(\lambda_{0}\right)=0$, there exists $a>0$ such that

$$
g(\lambda) \leq g\left(\lambda_{0}\right)+a\left(\lambda-\lambda_{0}\right)^{2}, \quad \forall \lambda:\left|\lambda-\lambda_{0}\right| \leq 1
$$

If $V^{\prime \prime}(t) \geq \lambda_{0}+1$, then (4.41) holds by taking $C$ large. If $\lambda_{0}<V^{\prime \prime}(t) \leq \lambda_{0}+1$, then by (4.40) and (4.42), we get

$$
\left(2 \sqrt{g\left(\lambda_{0}\right)}+\epsilon_{0}\right)^{2} \leq\left(z^{\prime}(t)\right)^{2} \leq 4 g\left(V^{\prime \prime}(t)\right) \leq 4 g\left(\lambda_{0}\right)+4 a\left(V^{\prime \prime}(t)-\lambda_{0}\right)^{2}
$$

thus

$$
4 \epsilon_{0} \sqrt{g\left(\lambda_{0}\right)}+\epsilon_{0}^{2} \leq 4 a\left(V^{\prime \prime}(t)-\lambda_{0}\right)^{2}
$$

This shows (4.41) with $C=\sqrt{a}\left[g\left(\lambda_{0}\right)\right]^{-\frac{1}{4}}$.

However, by (4.35), (4.38) and (4.41), we have

$$
\begin{aligned}
z^{\prime \prime}\left(t_{1}\right) & =\frac{1}{z\left(t_{1}\right)}\left(\frac{V^{\prime \prime}\left(t_{1}\right)}{2}-\left(z^{\prime}\left(t_{1}\right)\right)^{2}\right) \\
& \geq \frac{1}{z\left(t_{1}\right)}\left(\frac{\lambda_{0}}{2}+\frac{\sqrt{\epsilon_{0}}}{2 C}-\left(2 \sqrt{g\left(\lambda_{0}\right)}+\epsilon_{0}\right)^{2}\right) \\
& \geq \frac{1}{z\left(t_{1}\right)}\left(\frac{\sqrt{\epsilon_{0}}}{2 C}-4 \epsilon_{0} \sqrt{g\left(\lambda_{0}\right)}-\epsilon_{0}^{2}\right)>0
\end{aligned}
$$

provided that $\epsilon_{0}$ is taken small enough. This however contradicts (4.38) and (4.39). This proves (4.37). Note that we have also proved (4.41) for all $t \in\left[t_{0}, T^{*}\right)$. This together with (4.33) imply (4.32) with $\delta_{0}=\min \left\{\delta_{1}, \frac{\sqrt{\epsilon_{0}}}{C}\right\}$.

Case 2. Let $u_{0} \in \Sigma$ satisfy (1.27), (1.28), (1.31) and (1.32). As in Step 1, we see that the conditions $(1.27),(1.28),(1.31)$ and $(1.32)$ are respectively equivalent to

$$
\lambda_{0} \geq 0, \quad\left(z^{\prime}(0)\right)^{2} \geq 4 g\left(\lambda_{0}\right)=\frac{\lambda_{0}}{2}, \quad V^{\prime \prime}(0)<\lambda_{0}, \quad z^{\prime}(0) \leq 0 .
$$

We claim that

$$
z^{\prime \prime}(t)<0, \quad \forall t \in\left[0, T^{*}\right) .
$$

Note that by $(4.35)$, we have $z^{\prime \prime}(0)<0$. Assume by contraction that (4.44) does not hold. Then there exists $t_{0} \in\left(0, T^{*}\right)$ such that

and $z^{\prime \prime}\left(t_{0}\right)=0$. By (4.43), we have

$$
z^{\prime \prime}(t)<0, \quad \forall t \in\left[0, t_{0}\right)
$$

$$
z^{\prime}(t)<z^{\prime}(0) \leq-2 \sqrt{g\left(\lambda_{0}\right)}, \quad \forall t \in\left(0, t_{0}\right]
$$

Hence $\left(z^{\prime}(t)\right)^{2}>2 g\left(\lambda_{0}\right)$ which combined with (4.25) imply that

$$
g\left(V^{\prime \prime}(t)\right)>g\left(\lambda_{0}\right), \quad \forall t \in\left(0, t_{0}\right] .
$$

It follows that $V^{\prime \prime}(t) \neq \lambda_{0}$ for all $t \in\left(0, t_{0}\right]$, and by continuity, we have

$$
V^{\prime \prime}(t)<\lambda_{0}, \quad \forall t \in\left[0, t_{0}\right]
$$

By (4.35), we obtain

$$
z^{\prime \prime}\left(t_{0}\right)=\frac{1}{z\left(t_{0}\right)}\left(\frac{V^{\prime \prime}\left(t_{0}\right)}{2}-\left(z^{\prime}\left(t_{0}\right)\right)^{2}\right)<\frac{1}{z\left(t_{0}\right)}\left(\frac{\lambda_{0}}{2}-\frac{\lambda_{0}}{2}\right)=0
$$

which is absurd. Now, assume by contradiction that the solution exists globally forward in time, i.e., $T^{*}=\infty$. By (4.44), we see that

$$
z^{\prime}(t) \leq z^{\prime}(1)<z^{\prime}(0) \leq 0, \quad \forall t \in[1, \infty)
$$

This contradicts with the fact that $z(t)$ is positive. The proof is complete. 


\section{ACKNOWLEDGEMENT}

This work was supported in part by the Labex CEMPI (ANR-11-LABX-0007-01). V. D. D. would like to express his deep gratitude to his wife - Uyen Cong for her encouragement and support.

\section{REFERENCES}

[1] A. K. Arora, B. Dodson, and J. Murphy, Scattering below the ground state for the 2d radial nonlinear Schrödinger equation, Proc. Amer. Math. Soc. 148 (2020), no. 4, 1653-1663. $\uparrow 3$

[2] J. Bellazzini and L. Forcella, Dynamical collapse of cylindrical symmetric dipolar Bose-Einstein condensates, preprint, available at http://arxiv.org/abs/2005.02894v1. $\uparrow 4$

[3] A. De Bouard and R. Fukuizumi, Stability of standing waves for nonlinear Schrödinger equations with inhomogeneous nonlinearities, Ann. Henri Poincaré 6 (2005), no. 6, 1157-1177. $\uparrow 1$

[4] L. Campos, Scattering of radial solutions to the inhomogeneous nonlinear Schrödinger equation, Nonlinear Anal. 202 (2021), 112118. $\uparrow 2,6,7$

[5] L. Campos and M. Cardoso, Blow up and scattering criteria above the threshold for the focusing inhomogeneous nonlinear Schrödinger equation, preprint, available at http://arxiv.org/abs/2001.11613. $\uparrow 3,5$

[6] M. Cardoso, L. G. Farah, C. M. Guzmán, and J. Murphy, Scattering below the ground state for the intercritical non-radial inhomogeneous $N L S$, preprint, available at http://arxiv.org/abs/2007.06165. $\uparrow 3,4,6,7,11$

[7] T. Cazenave, Semilinear Schrödinger equations, Courant Lecture Notes in Mathematics, vol. 10, New York University, Courant Institute of Mathematical Sciences, New York; American Mathematical Society, Providence, RI, 2003. $\uparrow 1,2$, 6

[8] J. Chen, On a class of nonlinear inhomogeneous Schrödinger equation, J. Appl. Math. Comput. 32 (2010), no. 1, 237-253. $\uparrow 1$

[9] J. Chen and B. Guo, Sharp global existence and blowing up results for inhomogeneous Schrödinger equations, Discrete Contin. Dyn. Syst. Ser. B 8 (2007), no. 2, 357-367. 11

[10] Y. Cho, S. Hong, and K. Lee, On the global well-posedness of focusing energy-critical inhomogeneous NLS, J. Evol. Equ. (2020), available at http://doi.org/10.1007/s00028-020-00558-1. $\uparrow 3$

[11] V. D. Dinh, Blowup of $H^{1}$ solutions for a class of the focusing inhomogeneous nonlinear Schrödinger equation, Nonlinear Anal. 174 (2018), 169-188. $\uparrow 2,4,20,23,26,30$

[12] V. D. Dinh, Energy scattering for a class of the defocusing inhomogeneous nonlinear Schrödinger equation, J. Evol. Equ. 19 (2019), no. 2, 411-434. $\uparrow 3$

[13] V. D. Dinh, Scattering theory in weighted $L^{2}$ space for a class of the defocusing inhomogeneous nonlinear Schrödinger equation, to appear in Advances in Pure and Applied Mathematics, available at https://arxiv.org/abs/1710.01392. $\uparrow 2$

[14] V. D. Dinh, Energy scattering for a class of inhomogeneous nonlinear Schrödinger equation in two dimensions, J. Hyperbolic Differ. Equ. 18 (2021), no. 1, 1-28. $\uparrow 3$

[15] V. D. Dinh, A unified approach for energy scattering for focusing nonlinear Schrödinger equations, Discrete Contin. Dyn. Syst. 40 (2020), no. 11, 6441-6471. $\uparrow 3,5$

[16] B. Dodson and J. Murphy, A new proof of scattering below the ground state for the $3 D$ radial focusing cubic NLS, Proc. Amer. Math. Soc. 145 (2017), no. 11, 4859-4867. $\uparrow 2,3$

[17] T. Duyckaerts, J. Holmer, and S. Roudenko, Scattering for the non-radial 3D cubic nonlinear Schrödinger equation, Math. Res. Lett. 15 (2008), no. 6, 1233-1250. $\uparrow 13$

[18] T. Duyckaerts and S. Roudenko, Threshold solutions for the focusing 3D cubic Schrödinger equation, Rev. Mat. Iberoam. 26 (2010), no. 1, 1-56. $\uparrow 5$

[19] T. Duyckaerts and S. Roudenko, Going beyond the threshold: scattering and blow-up in the focusing NLS equation, Comm. Math. Phys. 334 (2015), no. 3, 1573-1615. $\uparrow 3,5$

[20] L. G. Farah, Global well-posedness and blow-up on the energy space for the inhomogeneous nonlinear Schrödinger equation, J. Evol. Equ. 16 (2016), no. 1, 193-208. $\uparrow 2,4,9$

[21] L. G. Farah and C. M. Guzmán, Scattering for the radial 3D cubic focusing inhomogeneous nonlinear Schrödinger equation, J. Differential Equations 262 (2017), no. 8, 4175-4231. $\uparrow 2,6,7,8,9,14$

[22] L. G. Farah and C. M. Guzmán, Scattering for the radial focusing inhomogeneous NLS equation in higher dimensions, Bull. Braz. Math. Soc. (N.S.) 51 (2020), no. 2, 449-512. $\uparrow 2,6,7,8,9,14,15,18,19,20$

[23] D. Fang, J. Xie, and T. Cazenave, Scattering for the focusing energy-subcritical nonlinear Schrödinger equation, Sci. China Math. 54 (2011), no. 10, 2037-2062. $\uparrow 9$

[24] G. Fibich and X. Wang, Stability of solitary waves for nonlinear Schrödinger equations with inhomogeneous nonlinearities, Phys. D 175 (2003), no. 1-2, 96-108. $\uparrow 1$

[25] D. Foschi, Inhomogeneous Strichartz estimates, J. Hyperbolic Differ. Equ. 2 (2005), no. 1, 1-24. $\uparrow 6$

[26] R. Fukuizumi and M. Ohta, Instability of standing waves for nonlinear Schrödinger equations with inhomogeneous nonlinearities, J. Math. Kyoto Univ. 45 (2005), no. 1, 145-158. $\uparrow 1$

[27] Y. Gao and Z. Wang, Below and beyond the mass-energy threshold: scattering for the Hartree equation with radial data in $d \geq 5$, Z. Angew. Math. Phys. 71 (2020), no. 2, Paper No. 52, 23. $\uparrow 3$

[28] F. Genoud and C. A. Stuart, Schrödinger equations with a spatially decaying nonlinearity: existence and stability of standing waves, Discrete Contin. Dyn. Syst. 21 (2008), no. 1, 137-186. $\uparrow 1,2$

[29] François Genoud, A uniqueness result for $\Delta u-\lambda u+V(|x|) u^{p}=0$ on $\mathbb{R}^{2}$, Adv. Nonlinear Stud. 11 (2011), no. 3 , 483-491. $\uparrow 2,28$

[30] R. T. Glassey, On the blowing up of solutions to the Cauchy problem for nonlinear Schrödinger equations, J. Math. Phys. 18 (1977), no. 9, 1794-1797. $\uparrow 22$

[31] C. D. Guevara, Global behavior of finite energy solutions to the d-dimensional focusing nonlinear Schrödinger equation, Appl. Math. Res. Express. AMRX 2 (2014), 177-243. $\uparrow 9,13,15$ 
[32] C. M. Guzmán, On well posedness for the inhomogeneous nonlinear Schrödinger equation, Nonlinear Anal. Real World Appl. 37 (2017), 249-286. $\uparrow 2,6,7$

[33] T. S. Gill, Optical guiding of laser beam in nonuniform plasma, Pramana 55 (2000), 835-842. 11

[34] L. Jeanjean and S. Le Coz, An existence and stability result for standing waves of nonlinear Schrödinger equations, Adv. Differential Equations 11 (2006), no. 7, 813-840. $\uparrow 1$

[35] M. Keel and T. Tao, Endpoint Strichartz estimates, Amer. J. Math. 120 (1998), no. 5, 955-980. $\uparrow 6$

[36] C. E. Kenig and F. Merle, Global well-posedness, scattering and blow-up for the energy-critical, focusing, non-linear Schrödinger equation in the radial case, Invent. Math. 166 (2006), no. 3, 645-675. 个2, 13

[37] C. S. Liu and V. K. Tripathi, Laser guiding in an axially nonuniform plasma channel, Physics of Plasmas 1 (1994), no. 9, 3100-3103. $\uparrow 1$

[38] Y. Liu, X. Wang, and K. Wang, Instability of standing waves of the Schrödinger equation with inhomogeneous nonlinearity, Trans. Amer. Math. Soc. 358 (2006), no. 5, 2105-2122. $\uparrow 1$

[39] Y. Martel, Blow-up for the nonlinear Schrödinger equation in nonisotropic spaces, Nonlinear Anal. 28 (1997), no. 12, 1903-1908. $\uparrow 4$

[40] F. Merle, Nonexistence of minimal blow-up solutions of equations $i u_{t}=-\Delta u-k(x)|u|^{4 / N} u$ in $\mathbf{R}^{N}$, Ann. Inst. H. Poincaré Phys. Théor. 64 (1996), no. 1, 33-85 (English, with English and French summaries). $\uparrow 1$

[41] C. Miao, J. Murphy, and J. Zheng, Scattering for the non-radial inhomogeneous NLS, to appear in Mathematical Research Letters, available at http://arxiv.org/abs/1912.01318. $\uparrow 3,11$

[42] B. Opic and A. Kufner, Hardy-type inequalities, Pitman Research Notes in Mathematics Series, vol. 219, Longman Scientific \& Technical, Harlow, 1990. $\uparrow 7$

[43] P. Raphaël and J. Szeftel, Existence and uniqueness of minimal blow-up solutions to an inhomogeneous mass critical NLS, J. Amer. Math. Soc. 24 (2011), no. 2, 471-546. $\uparrow 1$

[44] W. A. Strauss, Existence of solitary waves in higher dimensions, Comm. Math. Phys. 55 (1977), no. 2, $149-162 . \uparrow 25$

[45] C. Sulem and P. L. Sulem, The nonlinear Schrödinger equation, Applied Mathematical Sciences, vol. 139, SpringerVerlag, New York, 1999. Self-focusing and wave collapse. $\uparrow 1$

[46] T. Tao, On the asymptotic behavior of large radial data for a focusing non-linear Schrödinger equation, Dyn. Partial Differ. Equ. 1 (2004), no. 1, 1-48. $\uparrow 3$

[47] T. Tao, Nonlinear dispersive equations, CBMS Regional Conference Series in Mathematics, vol. 106, Published for the Conference Board of the Mathematical Sciences, Washington, DC; by the American Mathematical Society, Providence, RI, 2006. Local and global analysis. $\uparrow 1$

[48] J. F. Toland, Uniqueness of positive solutions of some semilinear Sturm-Liouville problems on the half line, Proc. Roy. Soc. Edinburgh Sect. A 97 (1984), 259-263. MR751198 个2, 28

[49] I. Towers and B. A. Malomed, Stable $(2+1)$-dimensional solitons in a layered medium with sign-alternating Kerr nonlinearity, J. Opt. Soc. Amer. B Opt. Phys. 19 (2002), no. 3, 537-543. $\uparrow 1$

[50] C. Xu and T. Zhao, A remark on the scattering theory for the $2 D$ radial focusing INLS, preprint, available at https://arxiv.org/abs/1908.00743. $\uparrow 3$

[51] E. Yanagida, Uniqueness of positive radial solutions of $\Delta u+g(r) u+h(r) u^{p}=0$ in $\mathbf{R}^{n}$, Arch. Rational Mech. Anal. 115 (1991), no. 3, 257-274. $\uparrow 2,28$

[52] S. Zhu, Blow-up solutions for the inhomogeneous Schrödinger equation with $L^{2}$ supercritical nonlinearity, J. Math. Anal. Appl. 409 (2014), no. 2, 760-776. $\uparrow 1$

(V. D. Dinh) Laboratoire Paul Painlevé UMR 8524, Université de Lille CnRS, 59655 Villeneuve D'Asce Cedex, France and Department of Mathematics, HCMC University of Education, 280 An Duong Vuong, Ho Chi Minh, VIETNAM

Email address: contact@duongdinh.com

(S. Keraani) Laboratoire Paul Painlevé UMR 8524, Université de Lille CNRS, 59655 Villeneuve D’Ascq Cedex, FRANCE

Email address: sahbi.keraani@univ-lille.fr 